\title{
Pulsed Photonuclear
}

\section{Assessment (PPA) Technique: CY 04 Year-End Progress Report}

J. L. Jones

W. Y. Yoon

K. J. Haskell

D. R. Norman

J. M. Zabriski

J. W. Sterbentz

S. M. Watson

J. T. Johnson

B. D. Bennett

R. W. Watson

K. L. Folkman

February 2005

Idaho National Engineering and Environmental Laboratory Bechtel BWXT Idaho, LLC 


\section{Pulsed Photonuclear Assessment (PPA) Technique: CY 04 Year-End Progress Report}

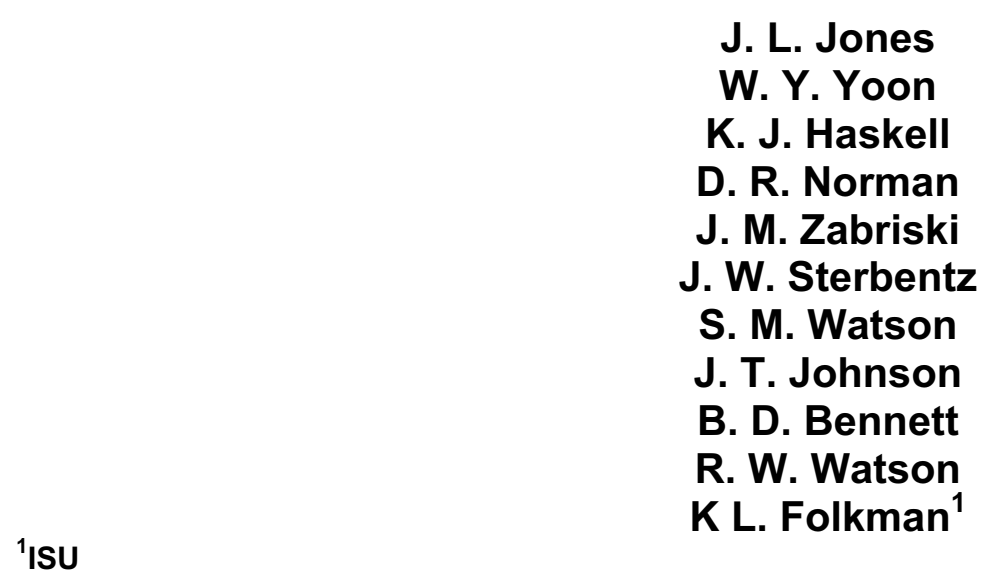

February 2005

Idaho National Engineering and Environmental Laboratory Idaho Falls, Idaho 83415

Prepared for the

U.S. Department of Energy

Assistant Secretary for Environmental Management

Under DOE Idaho Operations Office

Contract DE-AC07-99ID13727 


\begin{abstract}
Idaho National Laboratory (INL), along with Los Alamos National Laboratory (LANL) and Idaho State University's Idaho Accelerator Center (IAC), are developing an electron accelerator-based, photonuclear inspection technology for the detection of smuggled nuclear material within air-, rail-, and especially, maritime-cargo transportation containers. This CY04 report describes the latest developments and progress with the development of the Pulsed, Photonuclear Assessment (PPA) nuclear material inspection system, such as: (1) the identification of an optimal range of electron beam energies for interrogation applications, (2) the development of a new "cabinet safe" electron accelerator (i.e., Varitron II) to assess "cabinet safe-type" operations, (3) the numerical and experimental validation responses of nuclear materials placed within selected cargo configurations, 4) the fabrication and utilization of Calibration Pallets for inspection technology performance verification, 5) the initial technology integration of basic radiographic "imaging/mapping" with induced neutron and gamma-ray detection, 6) the characterization of electron beam-generated photon sources for optimal performance, 7) the development of experimentallydetermined Receiver-Operator-Characterization curves, and 8) several other system component assessments. This project is supported by the Department of Homeland Security and is a technology component of the Science \& Technology Active Interrogation Portfolio entitled "Photofission-based Nuclear Material Detection and Characterization."
\end{abstract}




\section{ACKNOWLEDGMENTS}

The authors want to express their sincere thanks to Dr. Frank Harmon, Mr. Chad O'Neill, and Mr. Brett King of Idaho State University for their outstanding support of our accelerator testing at the Idaho Accelerator Center complex and their skillful design and fabrication of the Varitron II electron accelerator. Finally, the continued comments, feedback, and contributions from Dr. Calvin Moss (Los Alamos National Laboratory) have been very helpful in the continued enhancement of this project. 


\section{CONTENTS}

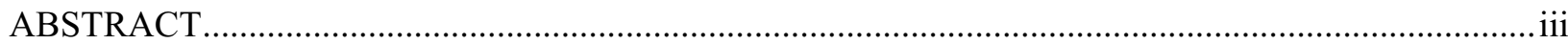

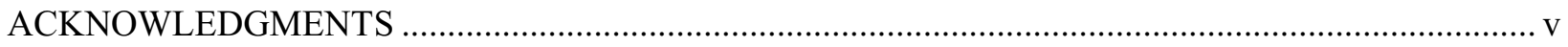

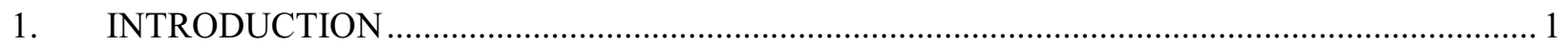

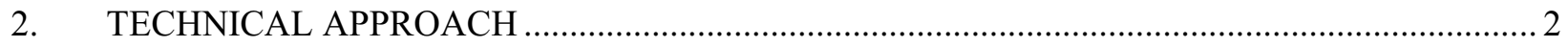

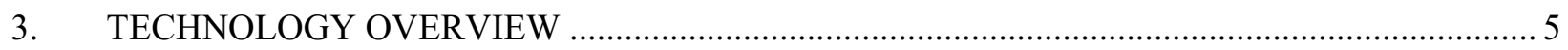

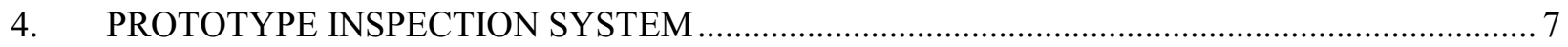

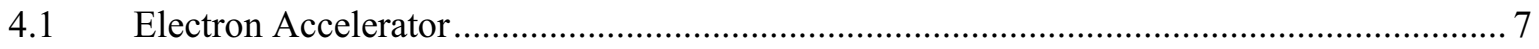

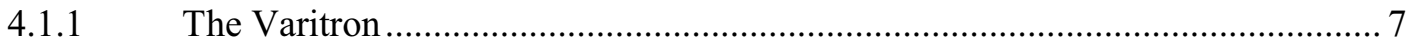

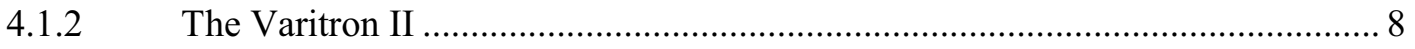

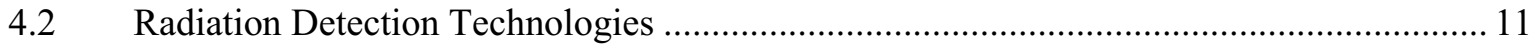

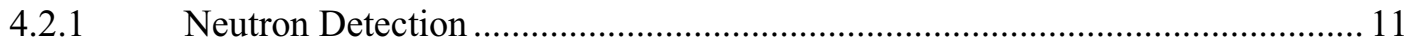

4.2.2 Gamma-ray Detection ............................................................................ 17

4.2.3 Gray-Scale "Imaging” for Low-/High-Z Material .......................................... 19

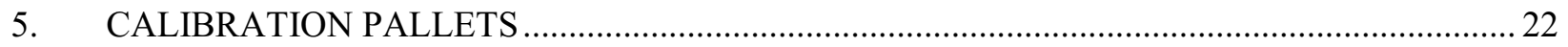

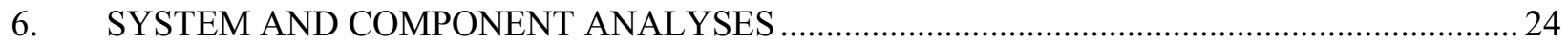

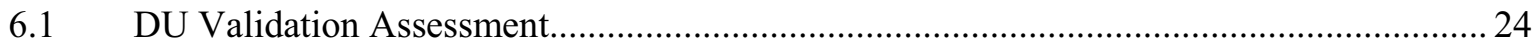

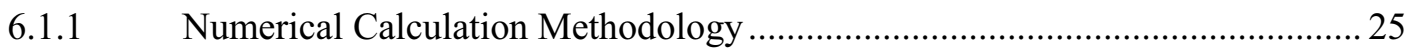

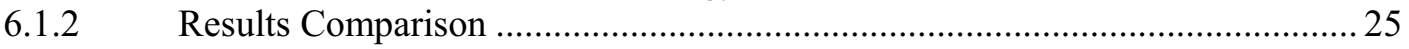

6.2 Receiver-Operator-Characteristic (ROC) Curves..................................................... 26

6.3 Electron Beam Energy Assessments ............................................................................ 28

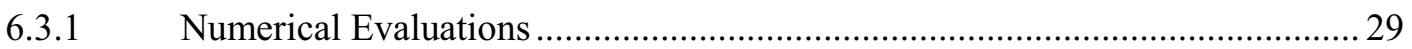

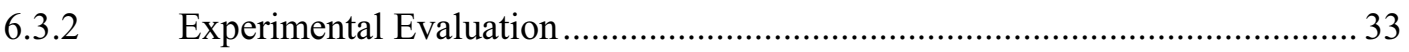

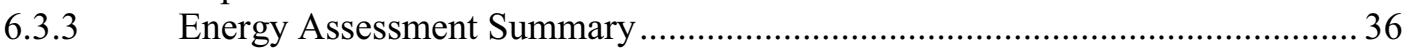

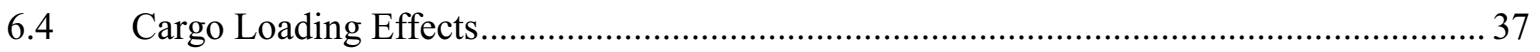

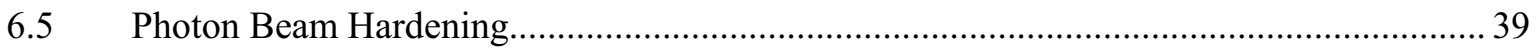

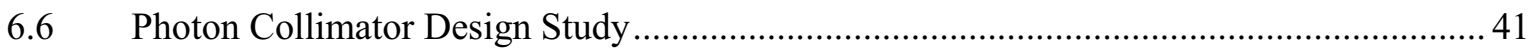

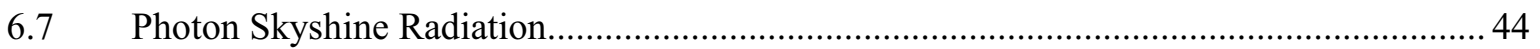

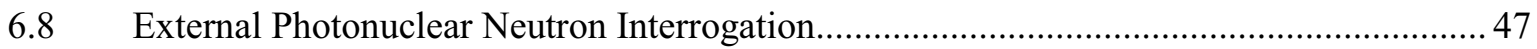




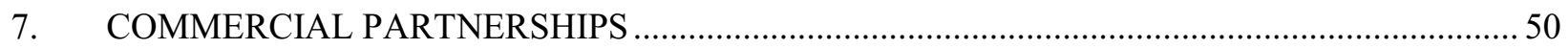

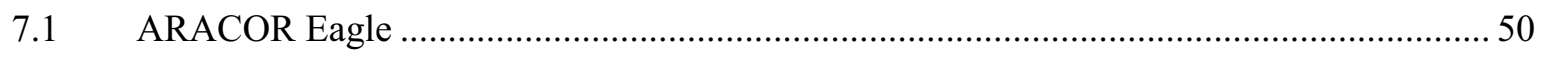

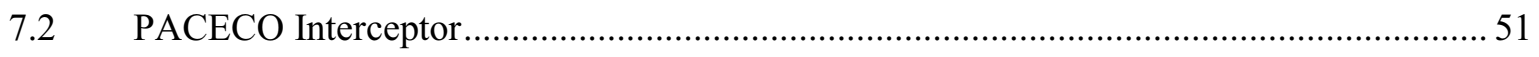

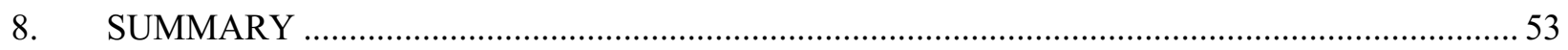

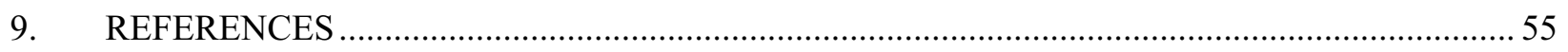

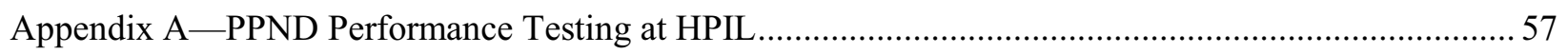

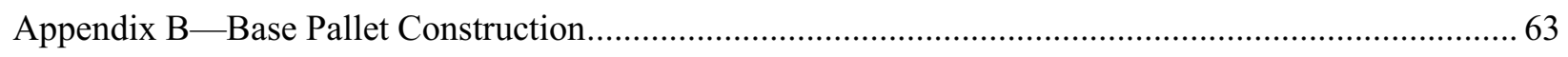

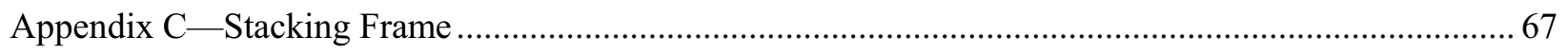

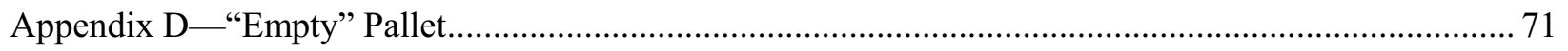

Appendix E-Celotex, Wood, Polyethylene and Borated-Polyethylene Pallet........................................ 75

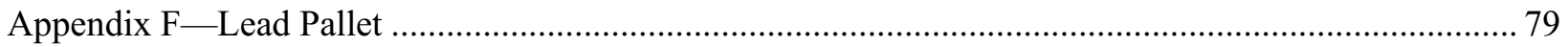

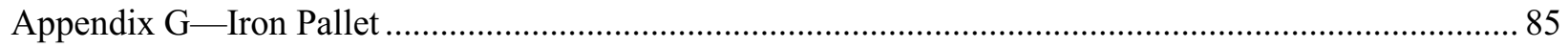

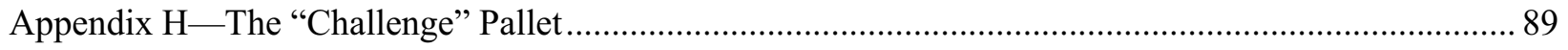




\section{Pulsed Photonuclear Assessment (PPA) Technique: CY04 Year-end Progress Report}

\section{INTRODUCTION}

For more than a decade Idaho National Laboratory (INL), along with Idaho State University's Idaho Accelerator Center (IAC), under the sponsorship of the Department of Energy's Office of Nonproliferation and National Security (NA22) and recently the Department of Homeland Security (DHS), have been developing high energy photon interrogation systems for various non-intrusive inspection applications. ${ }^{1-7}$ The primary focus for many of these applications has been in the detection of smuggled nuclear materials, especially highly enriched uranium (HEU), within various types of shielding configurations. During the last several years, and in collaboration with the IAC and Los Alamos National Laboratory (LANL), an energetic photon technology is being developed to address one of our homeland security's most important concerns - terrorism associated with the transportation of weapons of mass destruction (WMD), specifically weapons employing nuclear materials. While all aspects of cargo transportation are important and are being assessed, the focus of this report is nuclear material detection within maritime cargo containers and truck cargo since the these are the most common mode of commercial transportation. While many different active interrogation methods are being considered to address numerous aspects of this WMD inspection need, ${ }^{6}$ this paper presents the Calendar Year 2004 (CY04) progress of a photonuclear-based, nuclear material inspection technology identified as the Pulsed Photoneutron Assessment (PPA) technique. This report describes the basic technical approach, provides a technology overview, presents the numerical and experimental progress of the technology development, and highlights some commercial involvements. 


\section{TECHNICAL APPROACH}

Electron accelerators with energies below $12 \mathrm{MeV}$ have found considerable industrial and medical applications. Many of these accelerators, especially those below $8 \mathrm{MeV}$, are field transportable and are used for various radiography applications. Today, electron accelerators having even greater energy capabilities (up to 24-MeV) are common in most hospitals for medical oncology applications. These accelerators generate bremsstrahlung (i.e., energetic photons) having energies up to the maximum electron beam energy. The proposed active interrogation concept for this nuclear smuggling detection application leverages on these established accelerator applications and utilizes the ability to produce very penetrating photons to induce photofissions and neutron-induced fissions such as shown schematically in Figure 2-1. The system will be capable of not only detecting concealed/shielded nuclear material, but also allowing discrimination of HEU from other nuclear materials.

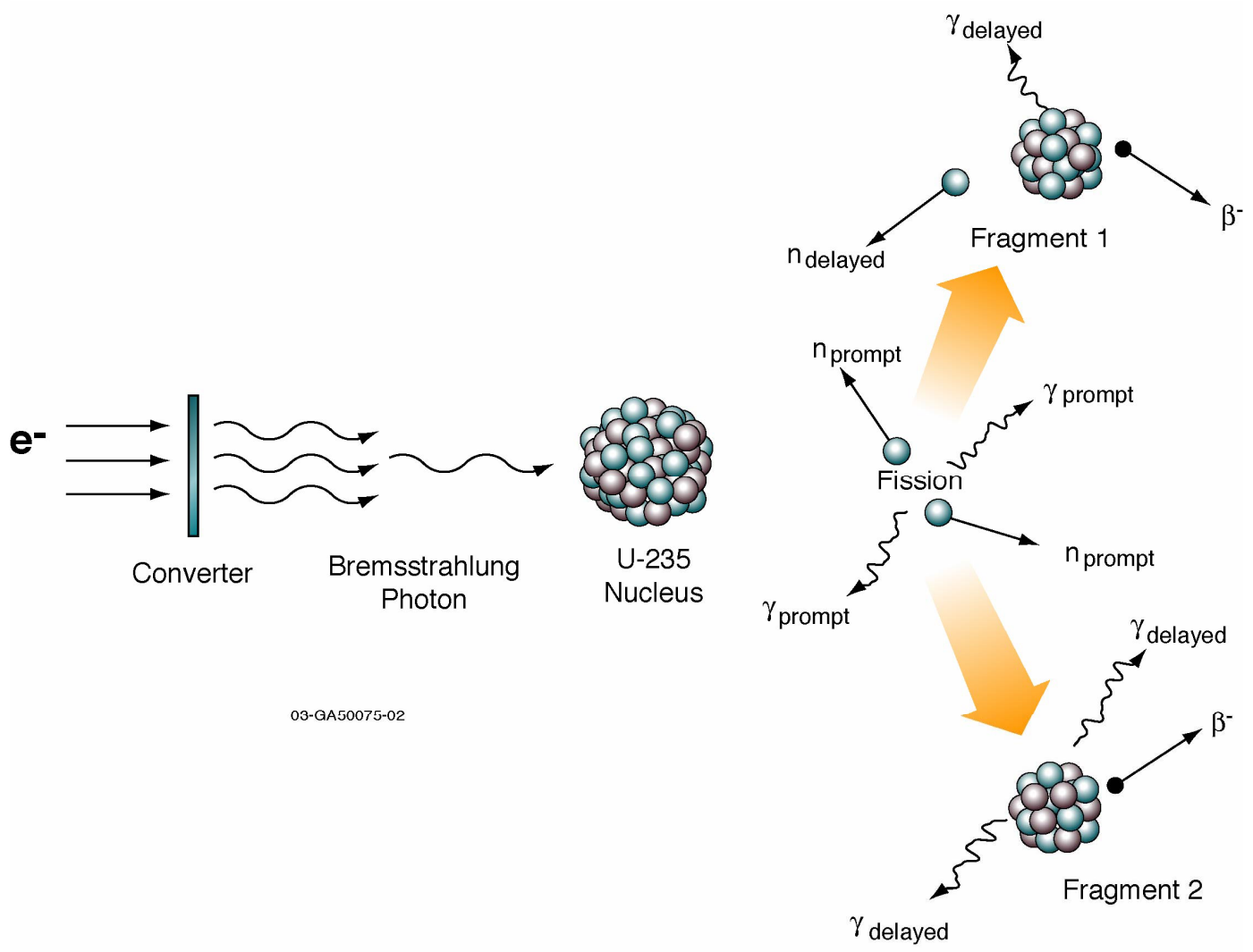

Figure 2-1. A schematic of the photofission process.

An electron accelerator will produce copious quantities of photons (i.e., $>\sim 10^{9}$ photons $/ \mathrm{s}$ ) and the resulting higher energy photons $(>6 \mathrm{MeV})$ are capable of penetrating through most shielding configurations. For example, better than 25 percent of 8 - and $12-\mathrm{MeV}$ photons will be transmitted through $2.66 \mathrm{~cm}$ and $2.44 \mathrm{~cm}$ of lead shielding, respectively, and more than 10 percent will penetrate through $4.42 \mathrm{~cm}$ and $3.98 \mathrm{~cm}$, respectively. These transmitted energetic photons, along with copious, multiple down-scattered photons, may then contribute to the desired photonuclear effects in the shielded nuclear materials.

Table 2-1 presents photonuclear threshold energies for many of the nuclear materials of interest in this assessment. These energies represent the minimum amount of photon energy required to induce a 
Table 2-1. Selected photonuclear threshold energies.

\begin{tabular}{lrrrc}
\hline \multicolumn{1}{c}{ Interaction Process } & U-235 & U-238 & Th-232 & Pu-239 \\
\hline$(\gamma, \mathrm{n})[\mathrm{MeV}]$ & 5.3 & 6.1 & 6.4 & 5.6 \\
$(\gamma$, fission $)[\mathrm{MeV}]$ & 5.8 & 5.8 & 6.0 & 5.6 \\
\hline
\end{tabular}

photonuclear reaction in that element. Neutrons from a photoneutron reaction $(\gamma, \mathrm{n})$ are emitted promptly $\left(\sim 10^{-12} \mathrm{~s}\right)$ after the reaction occurs. Photofission reactions, $(\gamma$,fission), emit both prompt and "delayed" gammas and neutrons. This "delay" occurs from the decay of the unstable fission fragments that can readily be detected between each accelerator-generated pulse of energetic photons. Nuclear materials have both a photoneutron and photofission interactions probability and the prompt neutrons will be slowed down and induce even more fissions in the nuclear material; the latter producing even more delayed radiation emissions. Due to the similarity of these threshold energies, especially for the photofission process, and based on the inherent capabilities of most commercial accelerator systems, it is extremely difficult to exploit these thresholds for material identification/discrimination. However, the detection of any gross delayed neutron emission is a direct indicator of the presence of a nuclear material.

Figures 2-2 and 2-3 show the total photonuclear yield and the photofission cross sections, ${ }^{8}$ respectively, for the nuclear materials of interest in this assessment. The total photonuclear yield cross section includes the photofission contribution. Of particular note are the photofission cross section differences in Figure 2-3 for each element beginning at approximately $8 \mathrm{MeV}$. Many of the energetic, induced photoneutrons, fission neutrons and delayed neutrons will escape the shielded configurations and may be detected. Nuclear material identification is possible by comparing the delayed neutron emissions per beam coulomb measured at different electron beam energy operations. ${ }^{9}$

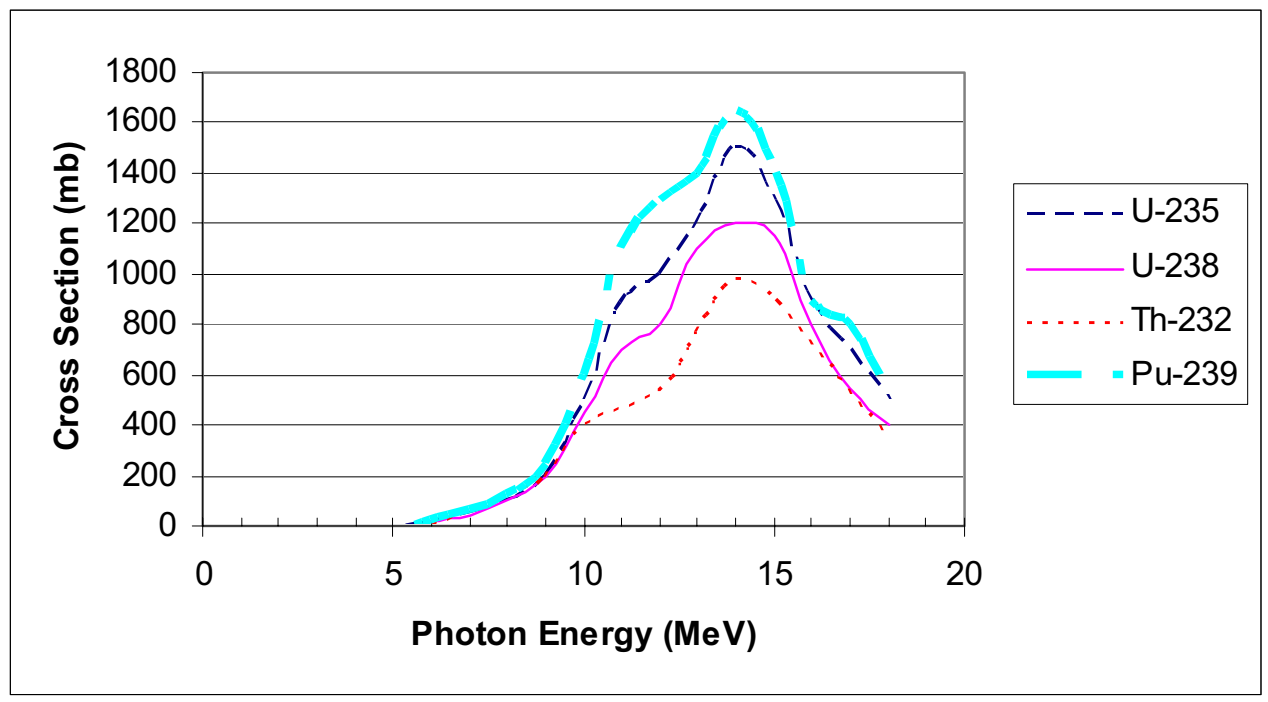

Figure 2-2. Total photonuclear yield cross sections for selected nuclear isotopes. 


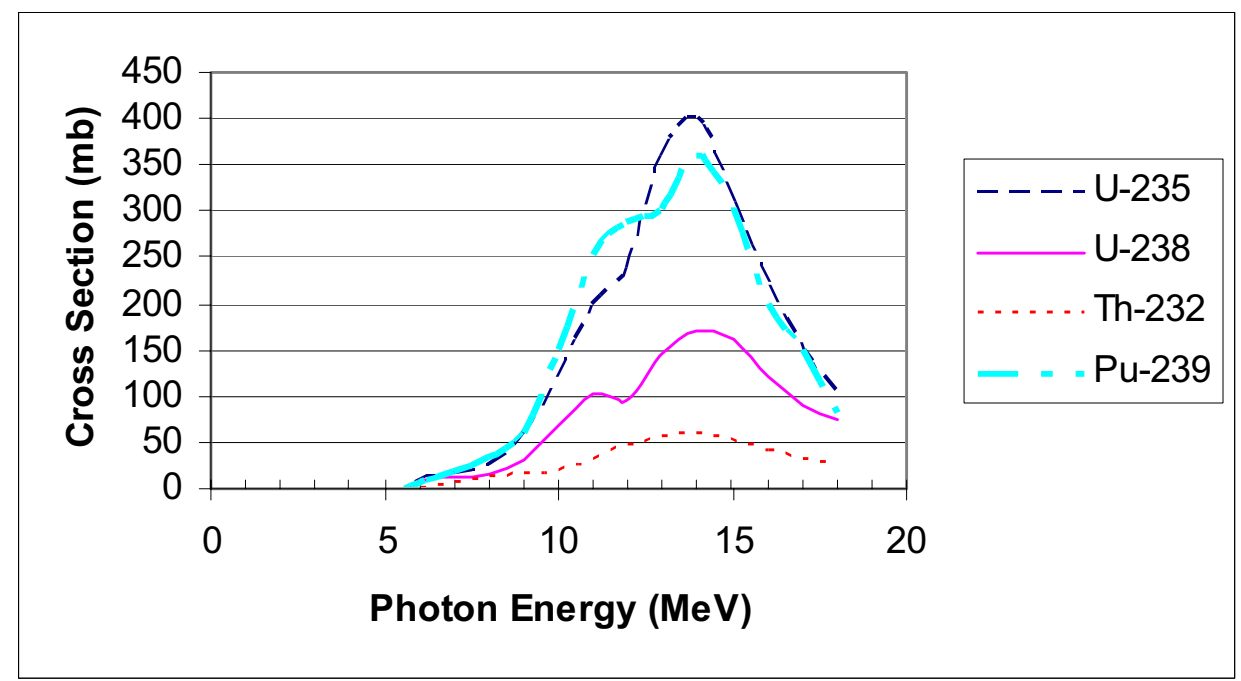

Figure 2-3. Photofission cross sections for selected nuclear isotopes. 


\section{TECHNOLOGY OVERVIEW}

The PPA inspection system consists of an electron accelerator (such as the INL Varitron ${ }^{2}$ ) to produce repeated pulses of high energy photons (i.e., bremsstrahlung), a neutron/gamma detection system capable of detecting the induced photonuclear emissions, and a gray scale-type "imaging" (or mapping) system. The initial prototype PPA system being used in assessing this technology performance with cargo container applications can be seen in Figure 3-1 with a fixed-site, truss-mounted detection system and the 2-m long Varitron (the "box" located in the right hand corner). A mobile, lift-type version of the detection system is shown in Figure 3-2 deployed on one side of a typical cargo container. The Varitron is a mobile accelerator system capable of generating pulses of 2-12 MeV electrons at rates up to $1 \mathrm{kHz}$ with an on-board, electron beam current and beam energy monitoring capability. The current prototype detection system is based on the patent-pending, 117-cm tall, 16-kg, INL-designed Pulsed Photonuclear Neutron Detector (PPND) ${ }^{3}$ design that differentiates prompt and delayed photon-induced neutron emissions between each accelerator pulse. Delayed neutron detection is a clear indication of the presence of nuclear material and additional data analysis and interrogations enable material identification. ${ }^{3}$ As is well known with any commercial x-ray radiography device, the more energetic the electrons used to produce photons within a high atomic number $(Z)$ converter, the more photons produced and the more energetic they can become. In addition, the resulting photon generation becomes more forward directed (i.e., along beam centerline) with increasing electron beam energies. This latter phenomenon allows increased interrogating fluxes with increased inspection distances as compared to conventional isotropic sources but may require electron beam rastering in actual cargo inspection applications. Since the higher photon energies enable both increased cargo penetration as well as increased photonuclear source generation, higher energy pulsed electron accelerators are more desirable and are only limited by photon/neutron dose, related personnel dose-shielding issues, and actively-induced backgrounds. While the present prototype system is assessing inspection performance using nominal 8- to $12-\mathrm{MeV}$ accelerator operations, this report considers induced emission responses with up to nominal $24-\mathrm{MeV}$ electron beam operations.

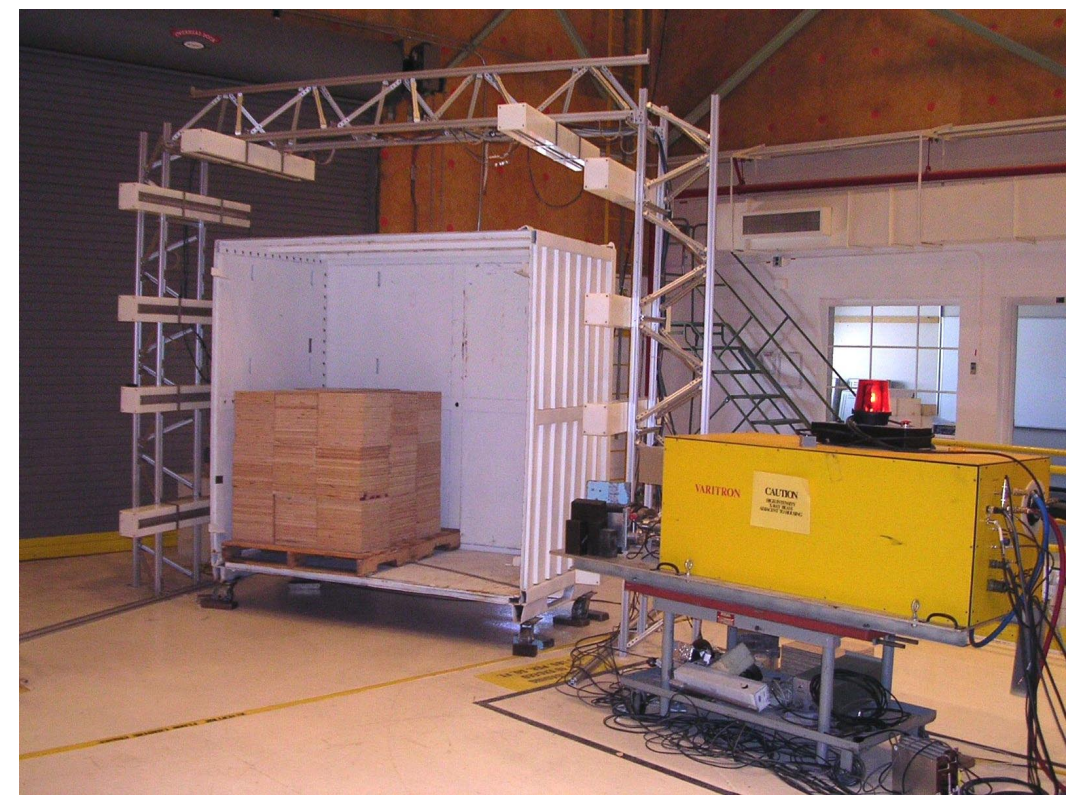

Figure 3-1. Fixed-site prototype PPA inspection configuration. 


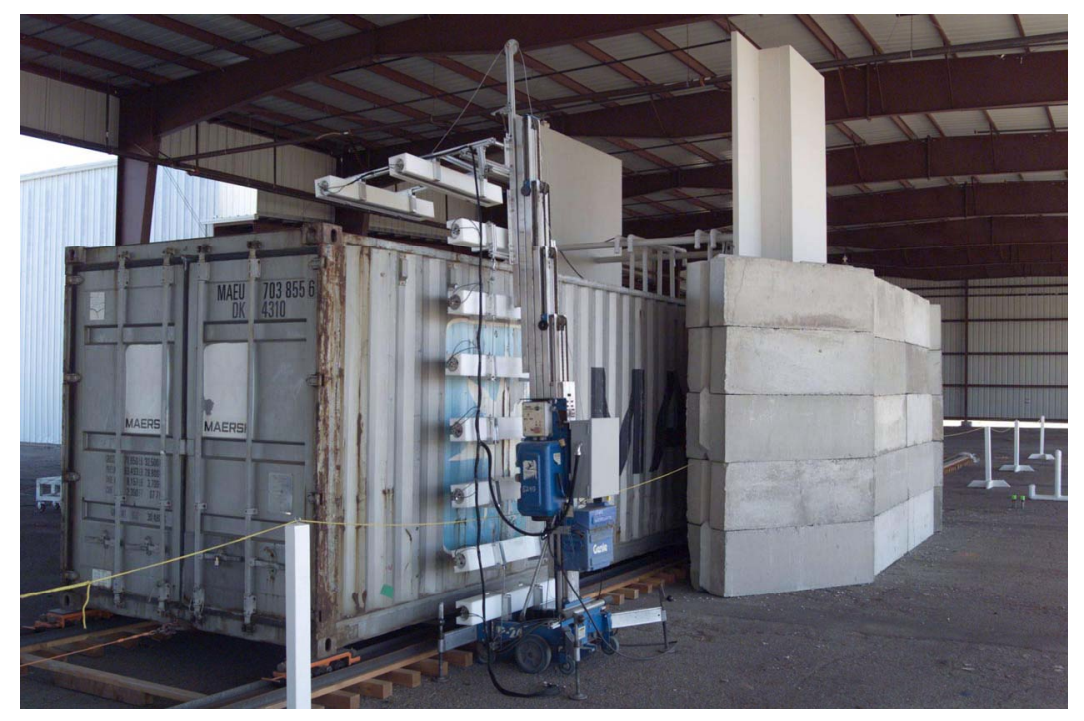

Figure 3-2. Mobile prototype PPA detection system deployment. 


\section{PROTOTYPE INSPECTION SYSTEM}

For a given cargo inspection, the PPA system consists of an electron accelerator as the interrogating photon (i.e., bremsstrahlung) source, an array of neutron and gamma-ray detectors, and a transmission-type, gray-scale "imaging" system. Each of these components is discussed below.

\subsection{Electron Accelerator}

The primary electron accelerator utilized this year was the INL Varitron; however, based on the recognized need to address radiation safety issues with any deployment of an energetic bremsstrahlung source, another electron accelerator (the Varitron II) has been designed and assembled. Initial Varitron II functionality tests were started in late December and operational characterization testing is planned for CY05.

\subsubsection{The Varitron}

The original INL Varitron, shown in Figure 4-1 with supporting components, is a unique, transportable, selectable-energy ( 2 to $12-\mathrm{MeV}$ ) electron accelerator capable of pulsing rates up to $1 \mathrm{kHz}$. The Varitron accelerator (without supporting components) is about 2-m long and about $76-\mathrm{cm}$ wide. The computer-interfaced, accelerator controls allow user-selection, control, and monitoring of various operational parameters including beam current to permit a high degree of operational repeatability. To allow selectable-energy operation, an on-board, magnetic spectrometer has been incorporated into the Varitron design to provide a kinetic energy characterization of the electron beam. Exchangeable tungsten collimators help define the spatial distribution of the forward-directed interrogating x-rays. Additional accelerator specifics can be found in Reference 2 .

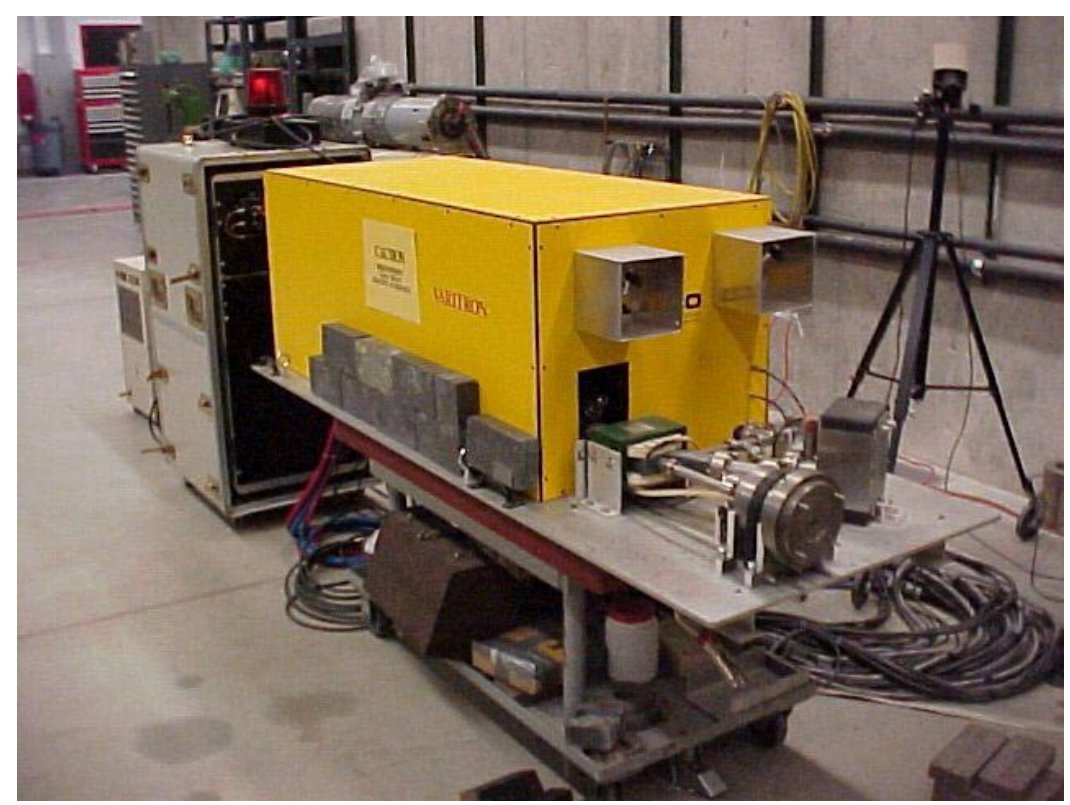

Figure 4-1. The INL Varitron. 


\subsubsection{The Varitron II}

The Variton II (shown in Figure 4-2) is a second-generation Varitron design that has been built and functionally tested at the IAC. As with the Varitron, this machine is built around a standard Varian L3000 accelerator guide and its radiofrequency (RF) components. This latest design allows it to have a "cabinet safe"-type operation, retain its transportability, have beam diagnostics similar to the original Varitron, and allow slightly higher beam energy operations. Based on preliminary initial testing, the Varitron II is already capable of energies of up to $\sim 11 \mathrm{MeV}$ with instantaneous beam currents of greater $30 \mathrm{~mA}$ (peak). The pulse width in the present configuration is three microseconds with a repetition rate of up to $300 \mathrm{~Hz}$. Additional modifications and testing are planned to tailor/optimize its overall performance, such as the use of a specialized klystron-driven power supply to allow even higher beam energies.

Several design changes with this machine will enable a more "cabinet safe" operation than the original Varitron. The L-3000 is now axial surrounded with a solenoid magnet. The massive $(\sim 70 \mathrm{~kg})$ solenoid magnet not only provides radiation shielding along the length of the guide itself but also enhances the capture of the low-energy electrons from the source resulting in higher application beam currents since a smaller fraction of the beam is lost. The latter also results in less conversion to bemsstrahlung radiation along the accelerator waveguide during the acceleration process. In addition, a massive ( $\sim 35 \mathrm{~kg})$ lead shield at the electron source end of the accelerator has been added (see Figure 4-3) to significantly attenuate the 180-degree-backscattered photon dose. Also, an aluminum box filled with lead surrounds the inline valve at the output end of the accelerator waveguide to reduce unwanted dose generation at this location.

Immediately next to the inline valve shielding (opposite to the accelerator waveguide and still on the accelerator beam centerline) will be a shielded beam diagnostic station. A new beam diagnostics tool, shown in Figure 4-4, is being developed at the IAC specifically for the Varitron II. A dipole electromagnet will bend the beam off axis into two faraday targets. These combined signals; along with information from the bending magnet; will provide accurate energy analysis and beam energy profiling with minimal system volume requirements. The latter is extremely important since the entire beam energy diagnostic station will be enclosed with shielding to control photon dose emissions.

Attached to the end of the beam energy diagnostic station and still centered on the beam centerline is the bremsstrahlung source that will also be surrounded by collimation shielding. While various bremsstrahlung sources are proposed, the Varitron II, unlike the Varitron I, will utilize a removable, air-cooled, electron/photon converter "cup" such as shown in Figure 4-5. This "cup" concept, containing an electron/photon converter, will enclose a $0.0025 \mathrm{~cm}$-thick stainless steel window that defines the end of the extended accelerator's vacuum chamber. A smaller, multiple version of this "cup" design is being fabricated for this accelerator to permit smaller collimator sizing. In addition, each "cup" design will provide a different converter thickness. This option allows testing with various electron/photon converter thicknesses ranging from high electron transmission designs to the medical designs that effectively stop all electron transmission. Various collimator designs are being considered and each is presented in Section 6.6. Figure 4-6 shows a representative collimator that has been tested and deployed with an inspection system developed for the PACECO Corporation (see Section 7.2) at the IAC.

The Varitron II is positioned on a wheeled stand that has been designed to enable the accelerator's beam centerline to be horizontally tilted as much as $\sim 40$ degrees up or $\sim 40$ degrees down. In addition, plans are underway to design an electron beam rastering station for the end of the accelerator to assess inspection coverage capability/requirements for various nuclear material placements. 


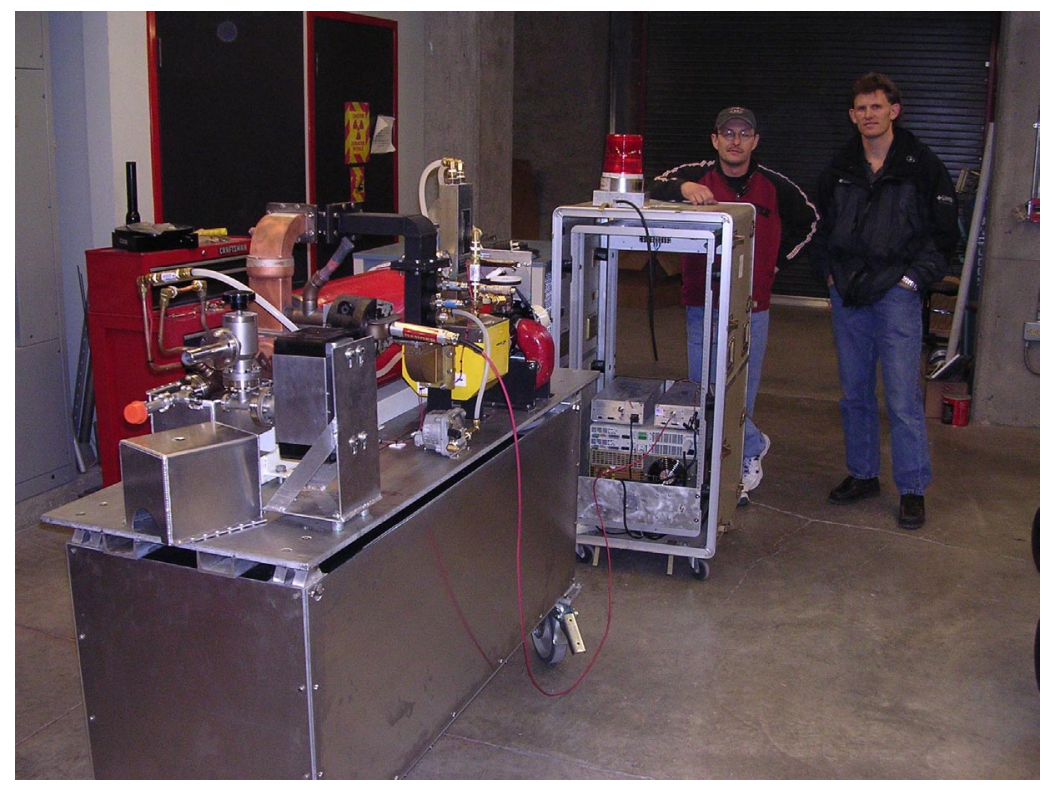

Figure 4-2. Varitron II with operational support components.

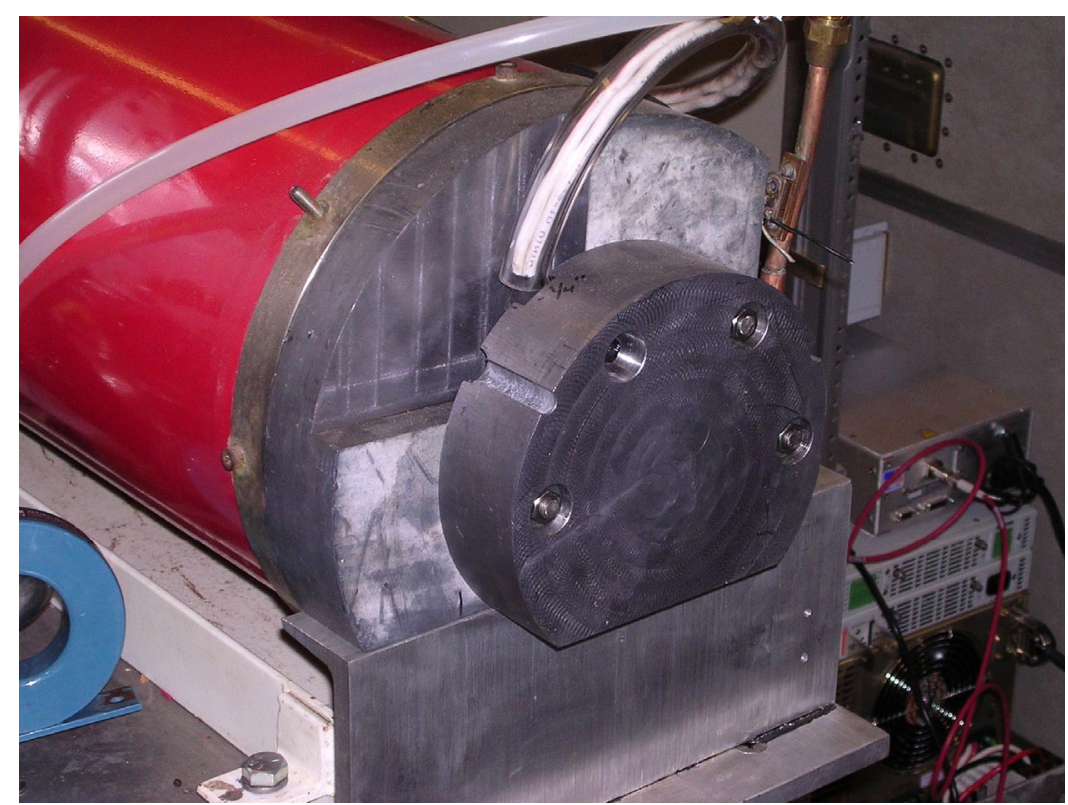

Figure 4-3. Accelerator's 180-degree backscatter shield (at the electron source end of the waveguide). 


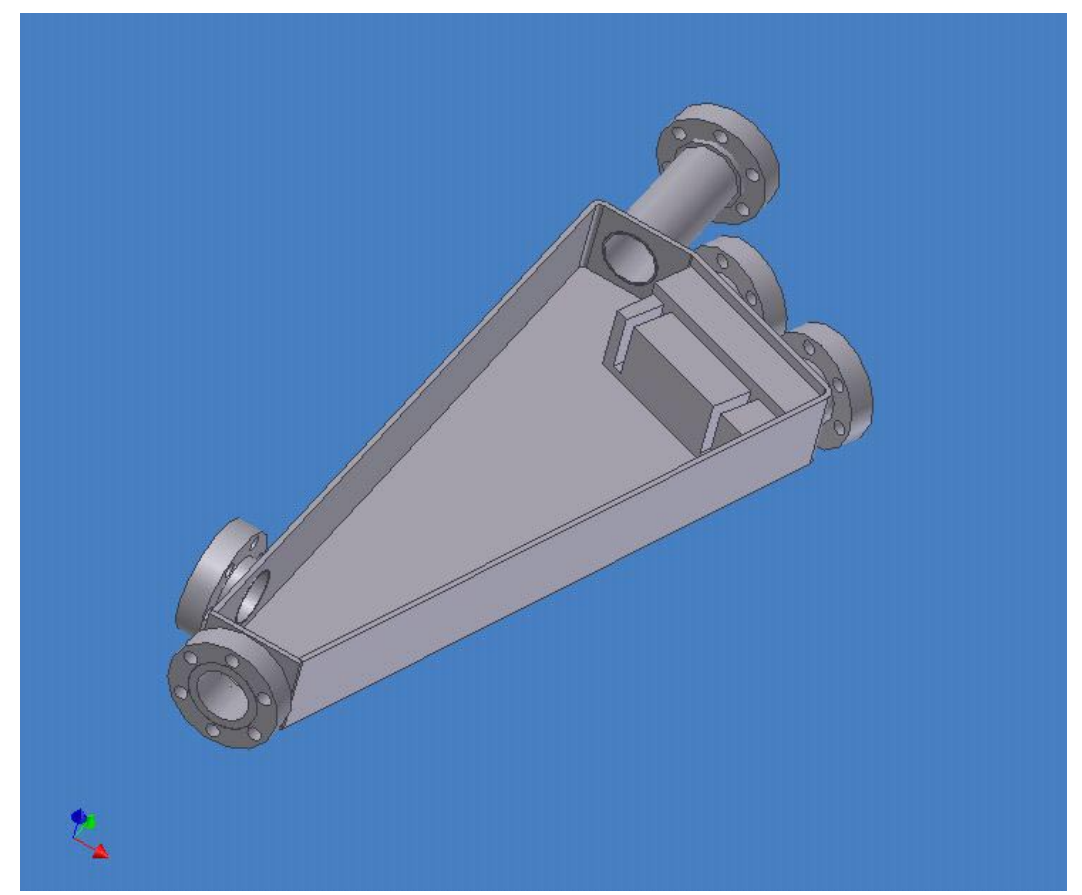

Figure 4-4. Beam energy diagnostics vacuum chamber.
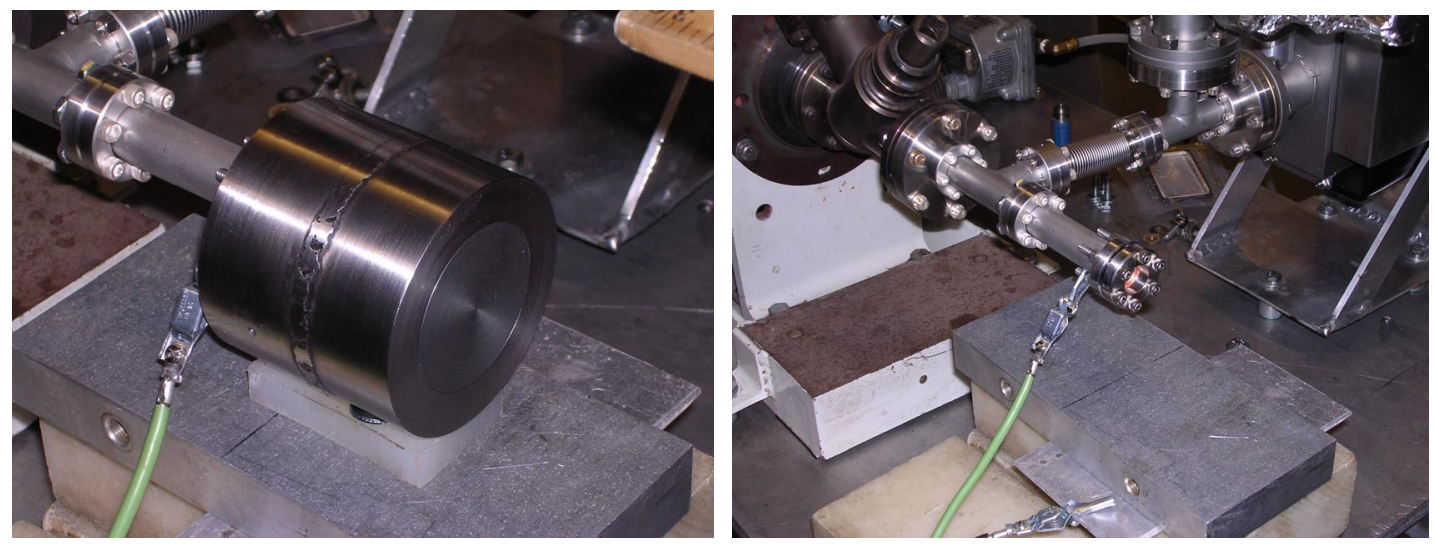

Figure 4-5. Large, prototypical, removable electron/photon converter (left). (Shown removed in right picture). 

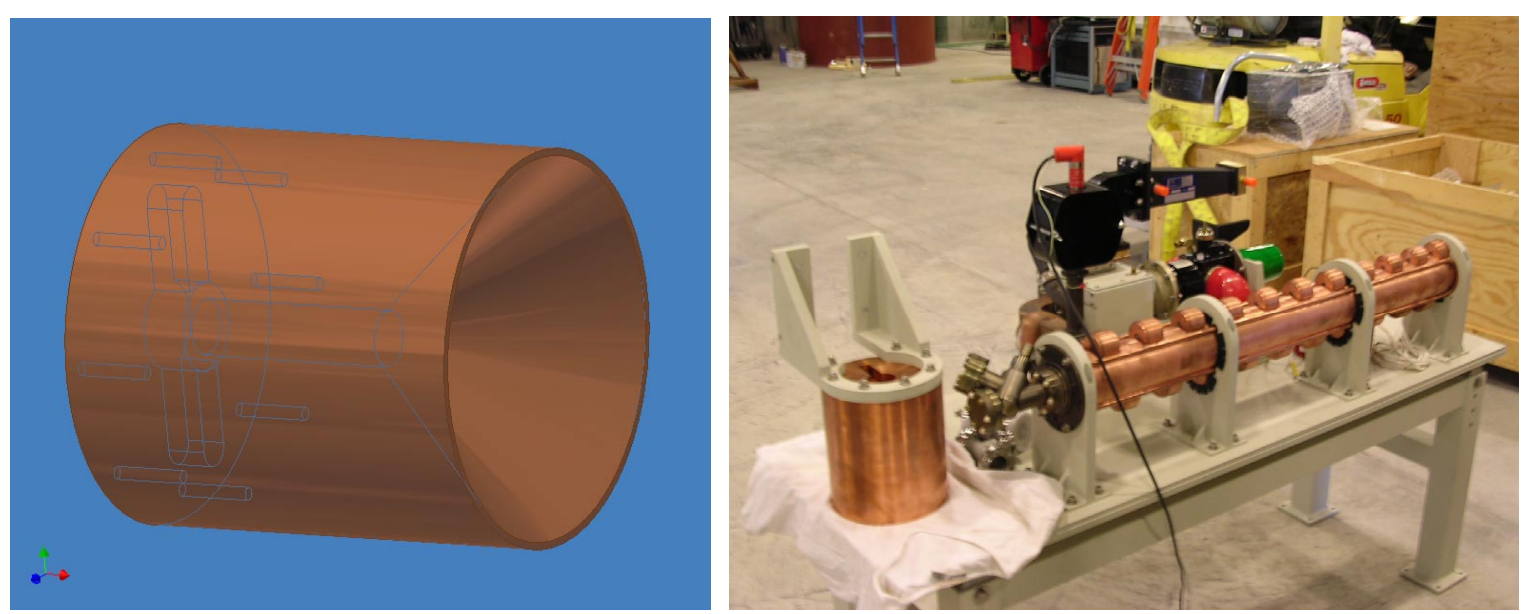

Figure 4-6. PACECO collimator (schematic left) and accelerator collimator and waveguide.

\subsection{Radiation Detection Technologies}

The PPA inspection system consists of prototype integrated neutron and gamma-ray nuclear material detection technologies with a transmission-type, gray-scale "imaging" (or mapping) system for high-Z (atomic number)/low-Z material detection.

\subsubsection{Neutron Detection}

The INL neutron detection system uses multiple, patent pending PPNDs, a personal computerbased commercial acquisition system, and mobile detection platform.

4.2.1.1 PPND. A PPND is shown in Figure 4-7 and a more complete description of the detector can be found in Reference 3. The PPND is a 16-kg, 117-cm long, 10.16-cm diameter neutron detector containing an internal high voltage power supply, an INL-built preamplifier, and a 10-atm., 2.54-cm diameter, ${ }^{3} \mathrm{He}$ tube surrounded by concentric rings of polyethylene moderator, cadmium metal, and highcontent, boron-loaded shielding. The internal preamplifier has been specially designed to operate within intense pulsed, photon-flash environments. The specific concentric ring design results in a very low sensitivity to room-return, low-energy neutrons typically associated with active interrogation environments and provides for the detection of $0.1-\mathrm{keV}$ to $1.0-\mathrm{MeV}$ neutrons that are representative of many prompt neutrons (occurring immediately after each accelerator pulse) and most delayed fission neutrons. In addition, the temporal detection response allows separation of the prompt and delayed neutron emissions. While future detection system development will also need to take advantage of the prompt neutron emissions that is at least two orders-of-magnitude greater than the delayed neutron emissions, the current focus of the neutron detection system is with the delayed neutrons. This delayed acquisition region is schematically presented in Figure 4-8 for a multi-pulsed photon interrogation.

A PPND has been calibrated with a californium-252 source to determine its total efficiency as approximately $1.6 \times 10^{-4}$ counts per californium-252 source fission neutron at 150 centimeters (See Appendix A). Similar efficiencies are observed with photonuclear neutron stimulation at 150 centimeters. With this detection efficiency, the PPND performs quite well and supports both passive and active detection applications. 


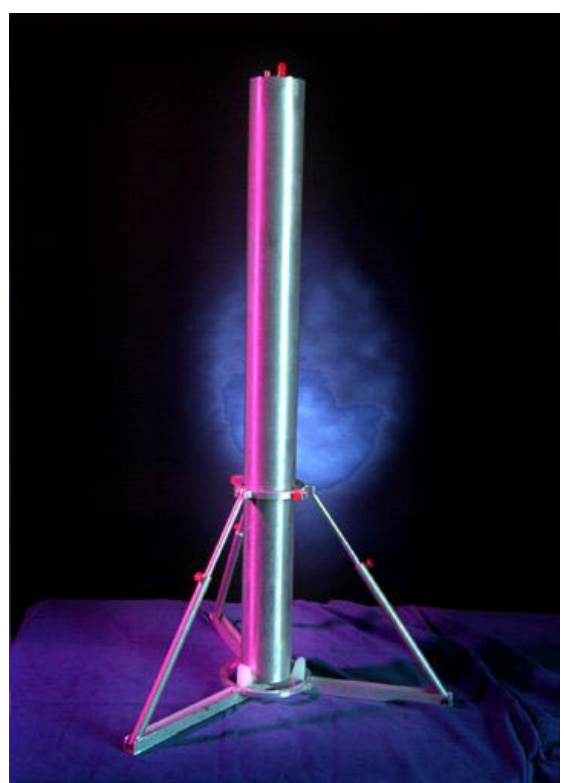

Figure 4-7. The Pulsed Photonuclear Neutron Detector (PPND).
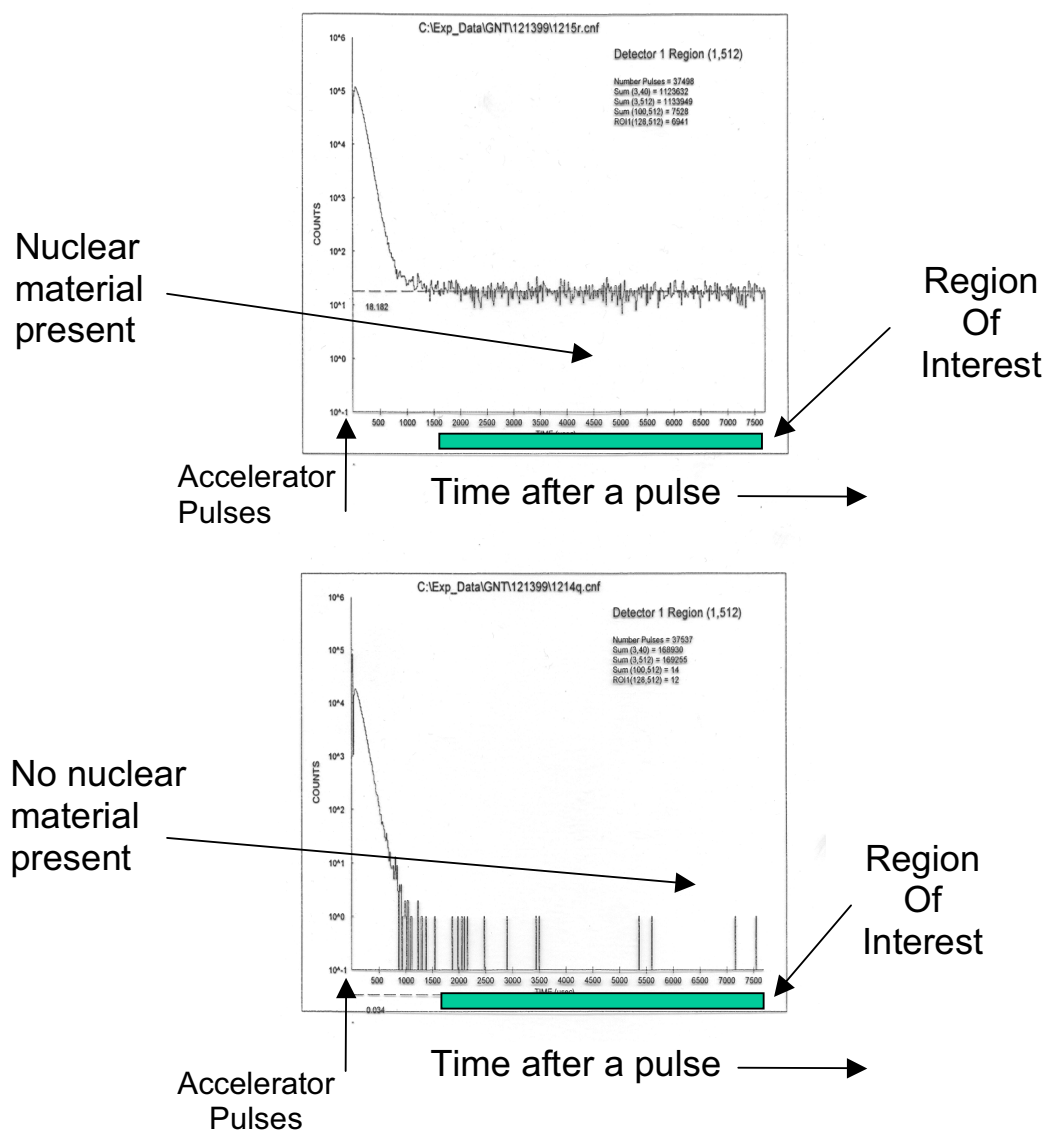

Figure 4-8. The delayed neutron "region-of-interest" identified in two temporal detector responses: one with nuclear material and one without. 
To reduce the natural cosmic neutron background contribution within the PPND for outdoor applications, a polyethylene shroud has been designed and fabricated. This $\sim 12.5 \mathrm{-kg}$ shroud is $15.24-\mathrm{cm}$ wide, $12.70-\mathrm{cm}$ deep, and $127-\mathrm{cm}$ tall (with two $2.54-\mathrm{cm}$ thick end caps) and has been designed to reduce the background effects by about 50-percent while maintaining a minimal added assembly weight. These shrouds are shown in Figure 4-9 without the standard end caps. These end caps do permit signal cable connections. Note to provide some detection directionality, the shrouds do not completely encase each PPND, but rather have been designed to allow some longitudinal PPND exposure; the outer edge of the 10.16-cm-diameter PPND is tangent to the outer edge of the 15.24-cm shroud width. This off-center placement of the PPND within the shroud (along with the 2.54-cm thick end caps) provides for at least a 2.54-cm shroud thickness on all sides except for front detector face.
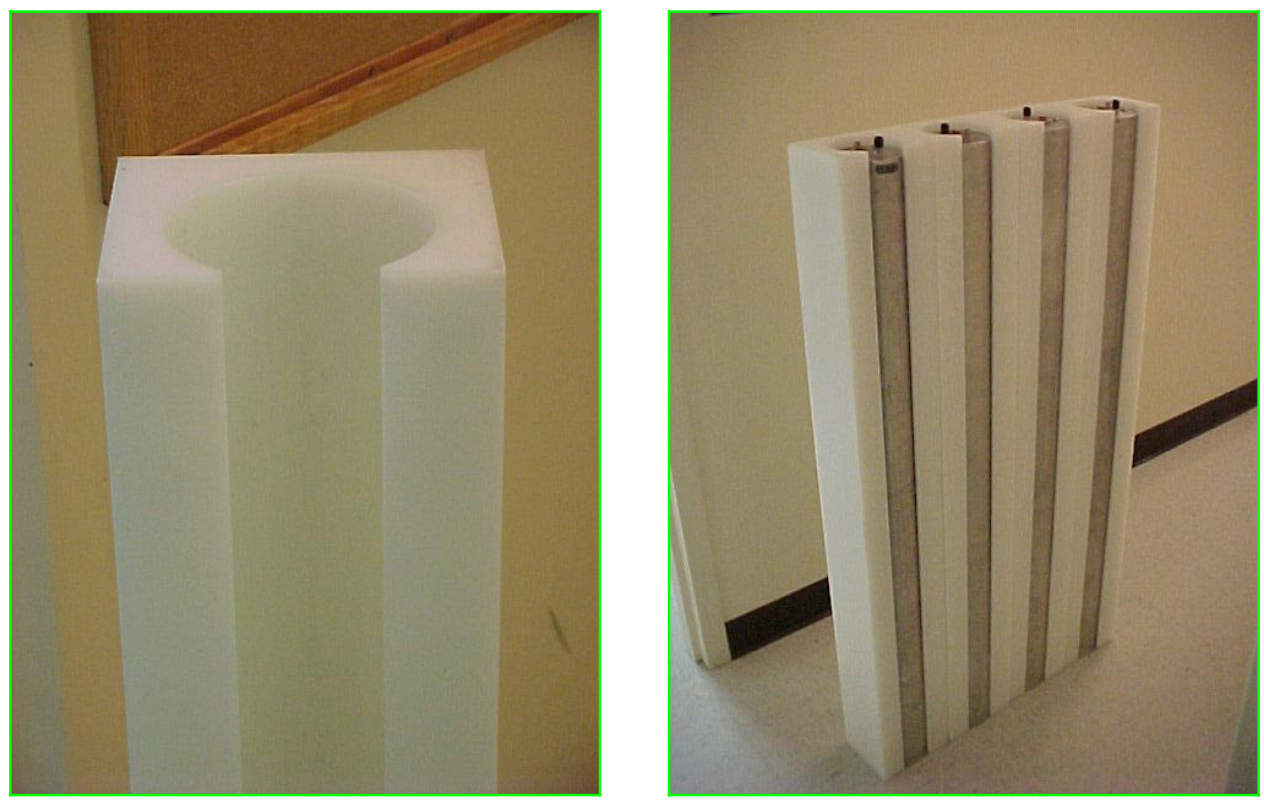

Figure 4-9. The PPND cosmic background suppression shroud (left) and PPNDs with shrouds.

4.2.1.2 Acquisition System. A multi-channel scaler electronic module, controlled by a personal computer-based data acquisition system (i.e., Genie-2000 software) and the accelerator's pulse trigger signal, allows the accumulation of all neutron counts after each accelerator pulse. The data is saved to the computer's hard drive. All data is acquired in 512 bins (note, the first bin records the number of accelerator pulses during the acquisition) with each bin typically being $15-\mu$ s wide for a $125-\mathrm{Hz}$ inspection application. By integrating the counts from selected bins, a user-selected "region-of-interest" count is identified. The delayed neutron region for this report usually corresponds to channels 128 to 512 or 1.9 to $7.7 \mathrm{~ms}$ after each accelerator pulse.

While essentially performing the same acquisition process, a newer acquisition process is being developed which utilizes National Instruments instrumentation and LabView programming. Figure 4-10 and Figure 4-11 shows the National Instrument's acquisition instrument (PXI) and the INL-developed 


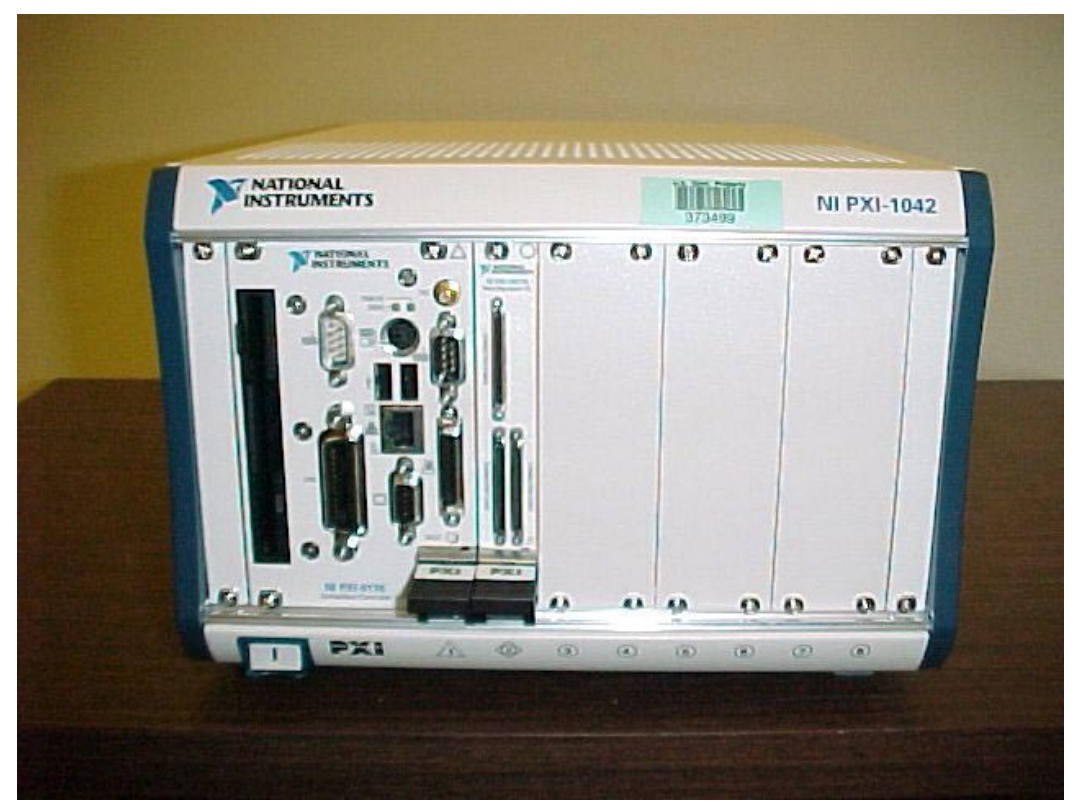

Figure 4-10. National Instrument's PXI system.

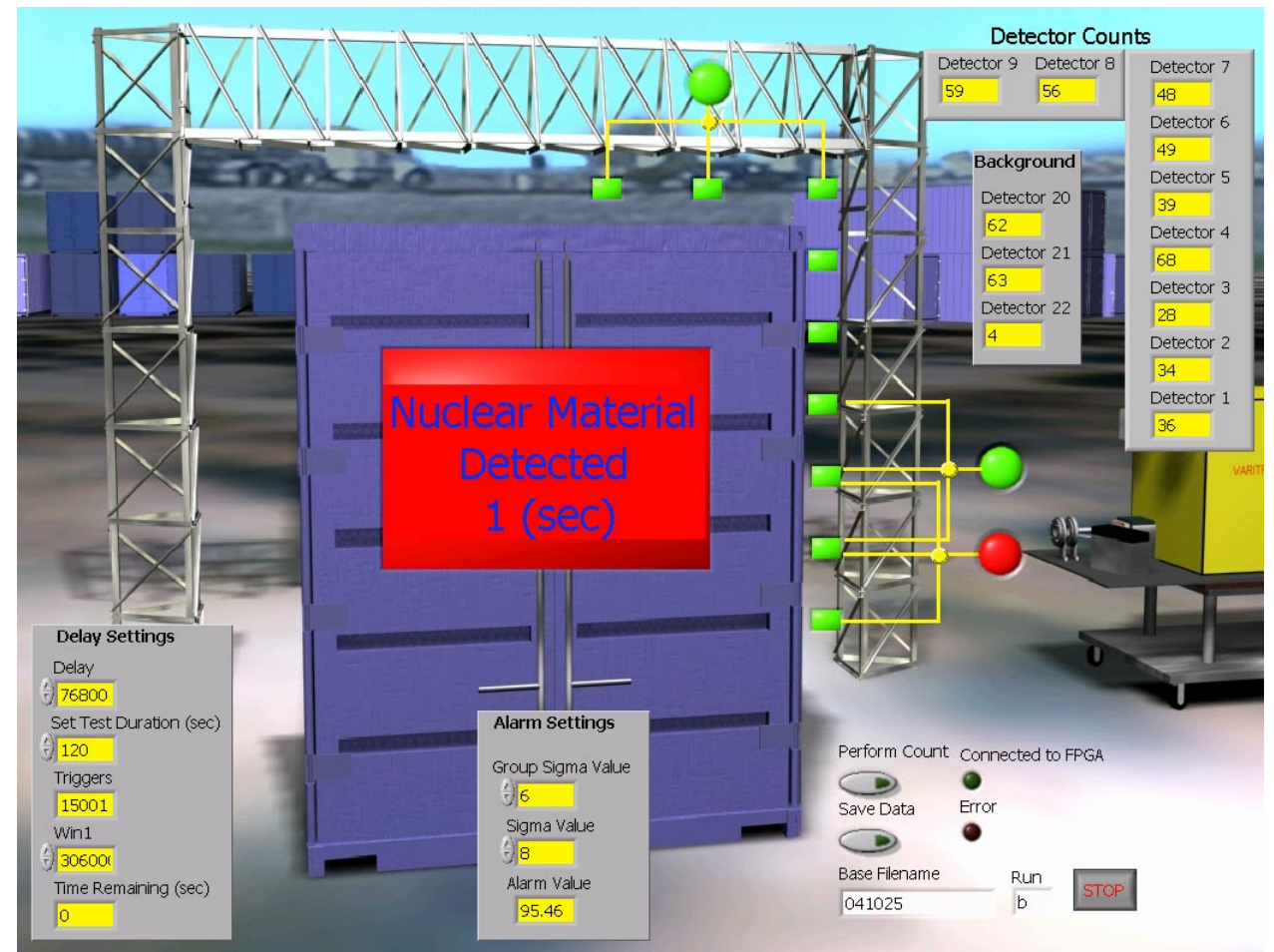

Figure 4-11. Typical Labview-based user interface display showing nuclear material detection (i.e., red). 
graphical user software interface, respectively. The system will provide increased bandwidth and a userfriendly interface. The PXI system contains a central processing unit in a rack-mountable chassis that allows for modular components to be installed on an as needed basis. The system currently contains a field programmable gate array (FPGA) module with reconfigurable input/output. This allows for embedded FPGA programs to be executed both in parallel and independent of CPU processes, enabling the overall system to operate uninterrupted in real-time. This method of operation provides continually processed calculations for increased detection rates as well as an increased overall system performance. By using this approach the user is provided with a simple "red light" alarm scheme for detection of nuclear material that can be operated by non-technical personnel. If needed, the PXI system can be powered from a 12-volt power supply.

4.2.1.3 Mobile Detector Mounting Platform. To support a very flexible and transportable PPA detector deployment capability, two standard Genie Man Lifts were purchased and modified to support multiple PPNDs. Figure 4-12 shows one of the two similar Genie Lifts. The two Genie Lifts have the ability to extend to a height of 9.75 and $7.32 \mathrm{~m}$, respectively. These lift heights can easily accommodate the proposed cargo- and truck-container inspection operations. Normally, each lift includes a singleperson bucket, four outrigger stabilizing legs (interconnected with the unit's safety interlock), front and rear wheels, a $110 \mathrm{VAC}$ auxiliary power outlet, a hydraulic system that utilizes a self-contained, hydraulic chain lift that is driven by a 12-volt power supply, a ground-based operator controlling station, and a 110 VAC operated battery charger.

The standard Genie Lift was modified to accommodate the variable placements of up to nine PPNDs (with cosmic background suppression shrouds) and to allow for lift-positioning close to an inspected cargo container. (Note: While this number of detectors and their placement has not been shown to be the optimal, this prototypical configuration does appear to enable adequate inspection coverage of a cargo container.) The "bucket" was removed, and the two front stabilizing legs were cut off, moved rearward, and reattached parallel to the main carriage assembly. The leg stabilizer interlock wiring was modified to facilitate this change and still maintain the integrity of the outrigger safety interlock system.

A mechanical cable system has been designed for both PPND position placements as well as defining the number of detectors that may be deployed. The INL-designed, mounting hardware, as shown in Figure 4-13, allows for easy in-field installation and removal of six of the nine detectors from the cabling. The lowest shrouded detector is mounted directly on the carriage assembly, and two highest shrouded detectors are firmly mounted on an assembly that is manually lowered over the top of the cargo container as shown in Figure 4-14. Figure 4-15 shows a cargo container with a deployed PPND detector lift.

Complete detector coverage of the cargo container during an inspection requires the use of a second identical Lift deployment system located on the opposite side of the container. The second completed, nine-detector lift is not shown in Figure 4-15. 


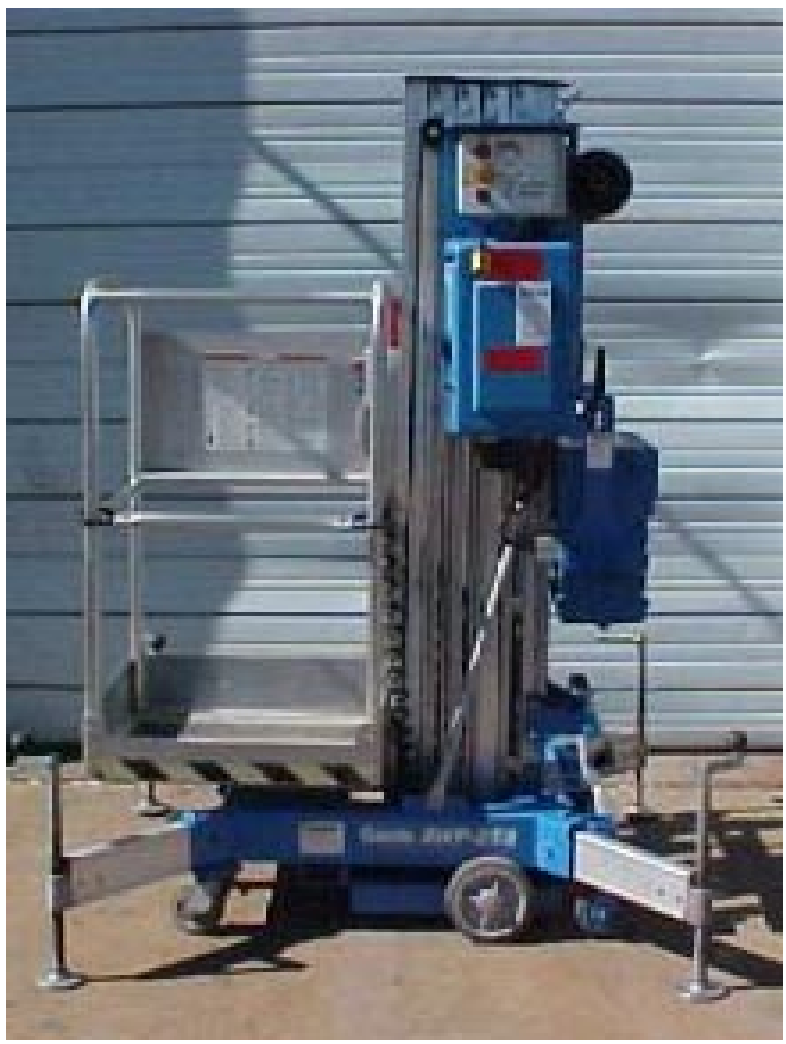

Figure 4-12. Standard Genie Lift.

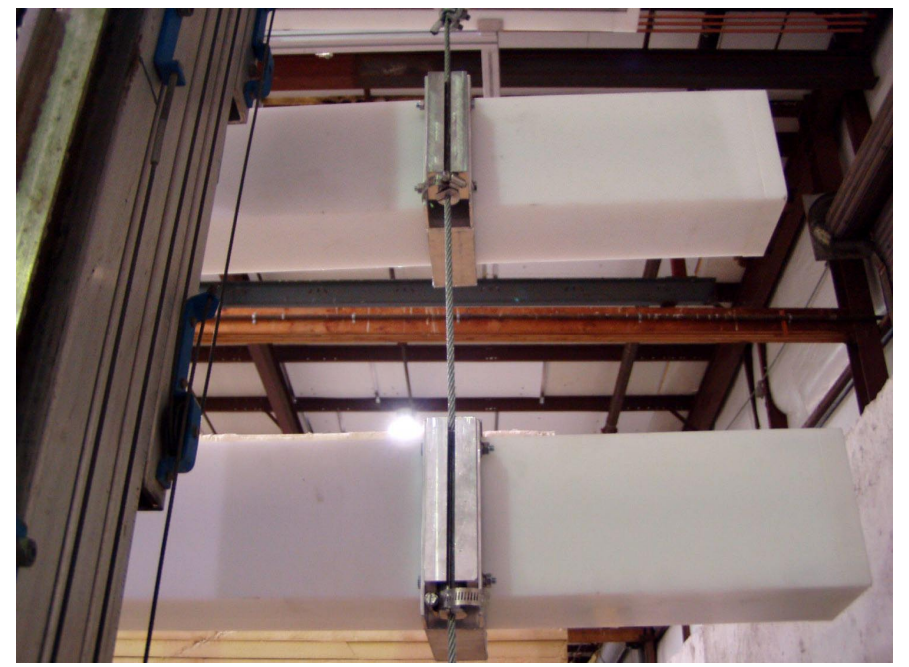

Figure 4-13. PPND and mounting hardware. 


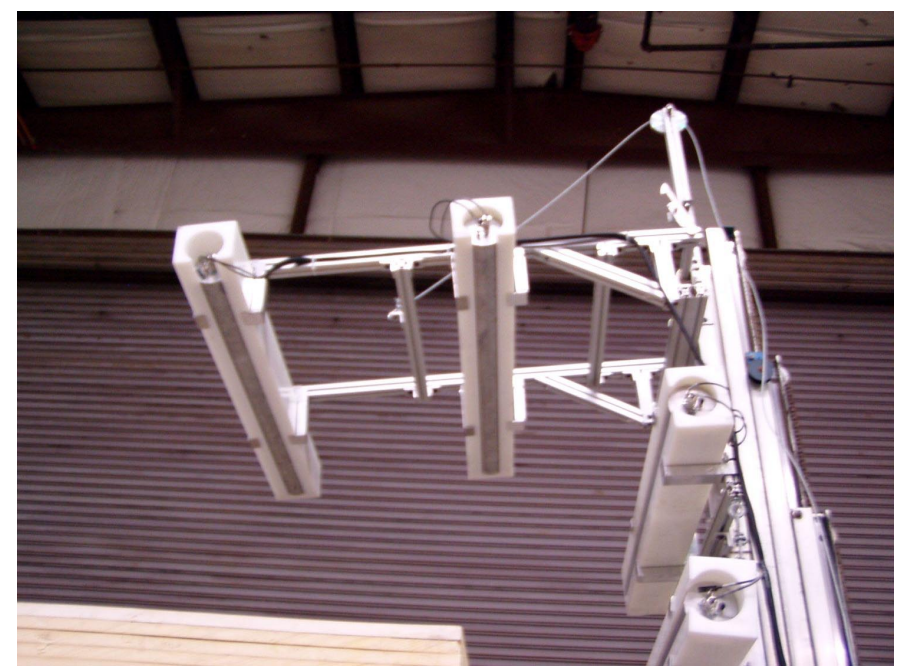

Figure 4-14. Two horizontal "over-the-top" detectors.

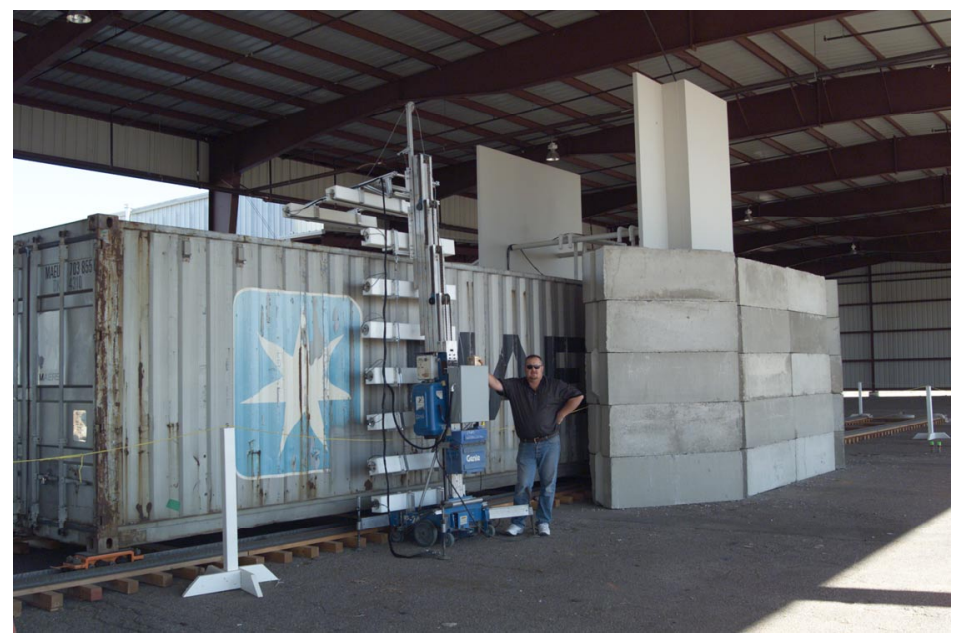

Figure 4-15. Deployed detection assembly Lift with a cargo container at the IAC.

\subsubsection{Gamma-ray Detection}

To support gamma-ray detection with the PPA system, the well-established Geiger-Muller (GM) detection technology is being investigated. Specifically, we are currently using $27.3-\mathrm{cm}$ long, $1.6-\mathrm{cm}$ diameter LND Model 719 tubes (containing 560 torr of neon and halogen) and plan to locate one with every PPND (see Figure 4-16). GM technology was chosen since these tubes tend to operate quite well within the pulsed photon flash environment presented by a PPA-type operation, are quite inexpensive, and require very little specialized electronics. Each tube requires only a high voltage $(\sim 900 \mathrm{~V})$ and a conventional front-end, signal processing electronics package to generate TTL-type counting output. For our intended applications we have elected to utilize existing PPND pre-amplifier electronics (with significant gain reduced amplification) and the 12-volt-operated, internal high voltage power module. Figure 4-17 shows the present GM tube design with its electronics package. In addition, Figure 4-18 provides two typical multi-scaler-type responses (with and without nuclear material present) for a $125-\mathrm{Hz}$, nominal $10-\mathrm{MeV}$ photon interrogation. For these responses, the nuclear material was placed approximately one meter from both the photon source and the gamma-ray detector. Additional detection response verification and optimization research is planned. 


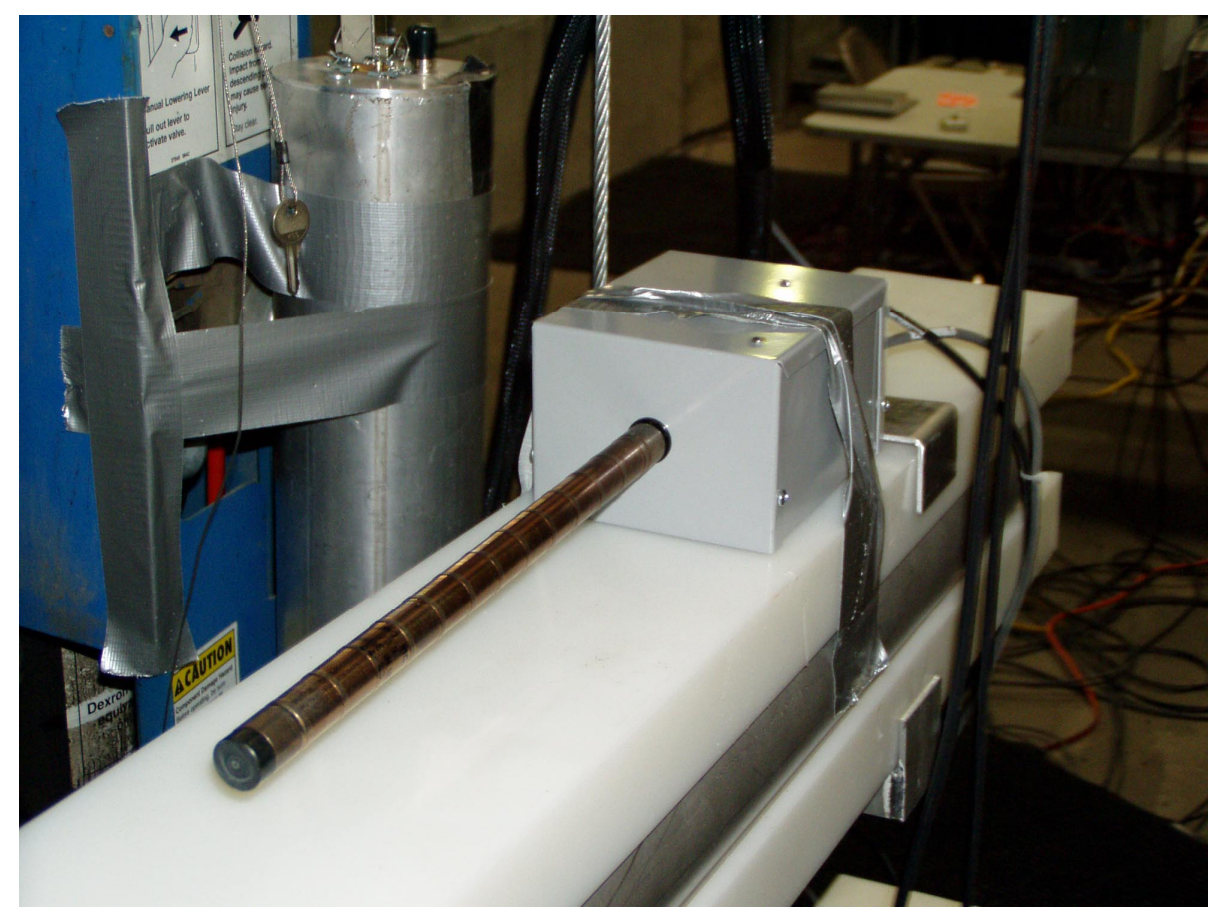

Figure 4-16. GM tube (with electronics base) located on top of a horizontally-mounted, shrouded PPND.

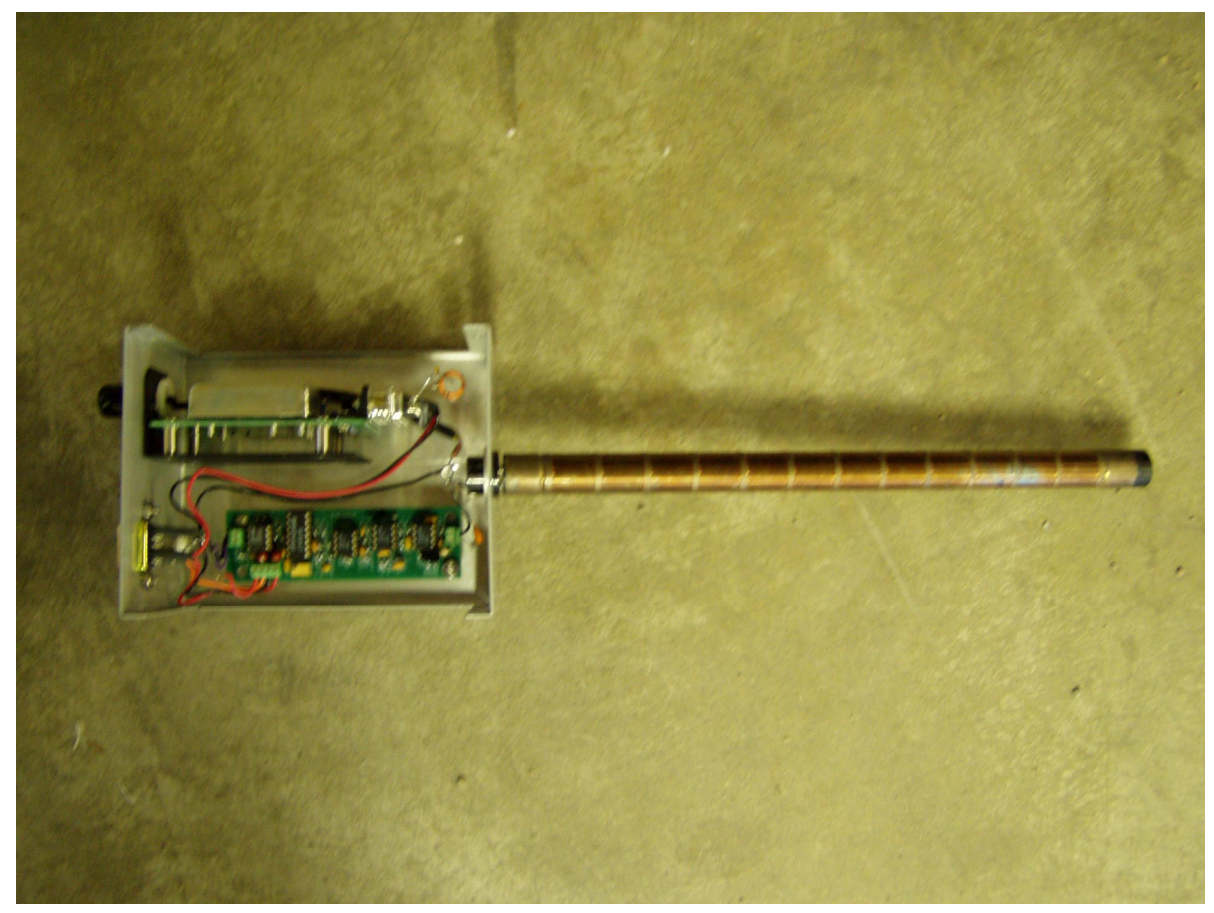

Figure 4-17. GM tube with its associated electronics box open. 


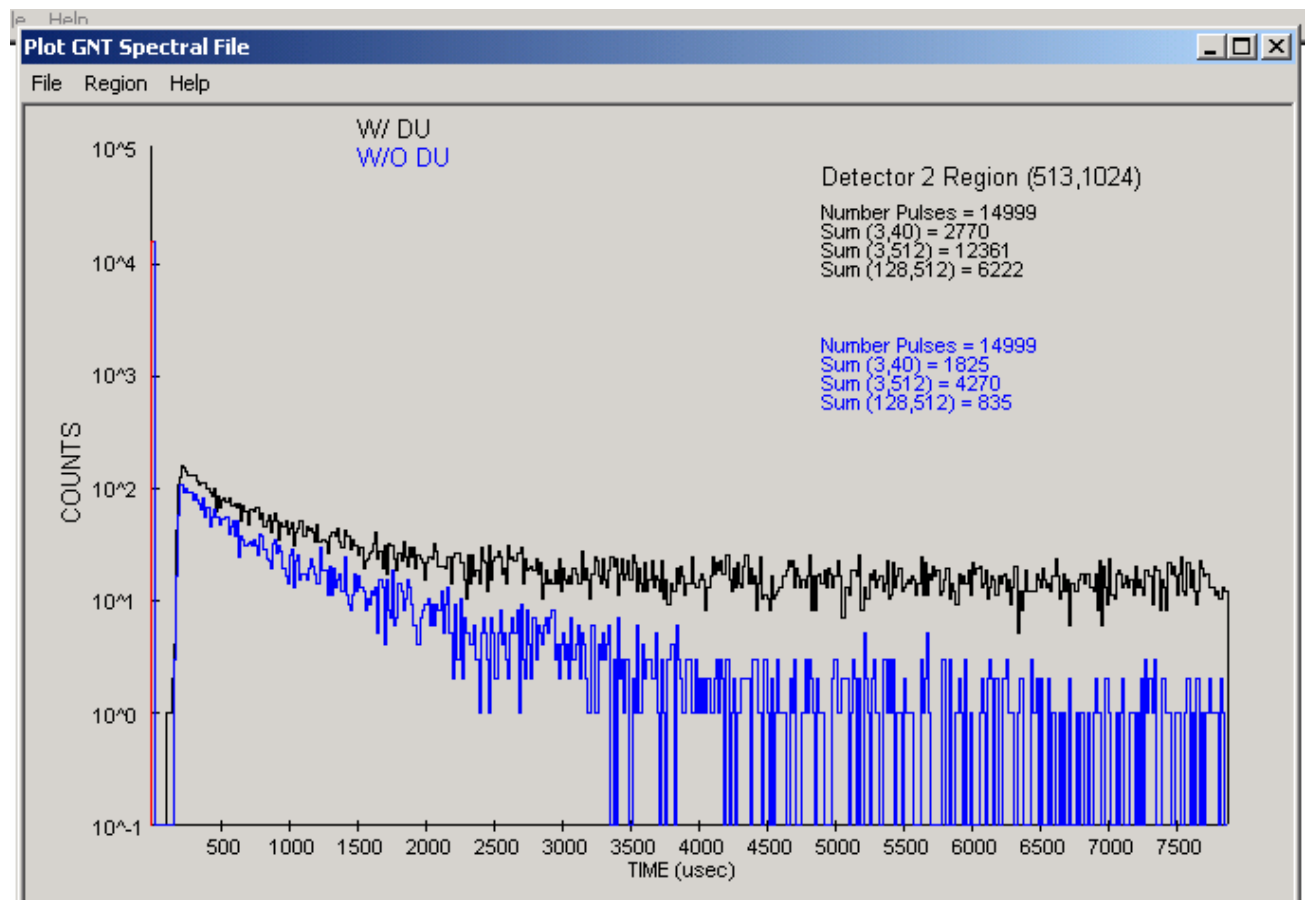

Figure 4-18. Temporal (time after each accelerator pulse) multi-scaler, GM detector output response for a nominal $10-\mathrm{MeV}$ photon interrogation with and without nuclear material (i.e., 4.8-kg depleted uranium).

\subsubsection{Gray-Scale "Imaging" for Low-/High-Z Material}

To assess the possibility of developing a very inexpensive and automatic (minimal user interaction requirement) gray-scale "imaging" (or mapping) system, an array of GM tubes is being developed. The tubes being used initially are the GM tubes described in the preceding section. However, in this case the tubes are not operated in the GM mode, but rather at a lower tube voltage (just below the "GM region") or in the "proportional region." This type of operation provides a signal that is proportional to the transmitted photons. Each tube is being provided with independent high voltage by an INL-built power supply consisting of multiple, regulated high-voltage power supplies (See Figure 4-19). The output of each tube for an empty cargo container configuration has a $\sim 100-\mu$ second recovery time. The analog signal is digitized and analyzed by a National Instrument's LabView software program to determine a gray-scale intensity. This software program is designed to provide a dark image when the photon source is very attenuated and a white version when the photon source is not attenuated. For example, Figure 4-20 (lower half) presents the "images" from a three-detector array having a transmission-type position relative to a various placements of a DU material surrounded by wood and interrogated with a nominal $10-\mathrm{MeV}$ operation. The darker "image" sections show high-Z (i.e., DU) detection.

Linear and grid-type "imaging" assembly arrays of proportional tubes are being constructed and tested. The specific array configuration will define the spatial resolution of the "imaging" system. Initial tests have begun using an array of three shielded and unshielded tubes as shown in Figure 4-21. These three tubes have shown very promising results in both a perpendicular and parallel orientation relative to the electron beam centerline. However, the need to have well-defined spatial resolution requires a parallel orientation with the beam. Based on the continued good performance of these proportional tubes, a prototype "imaging" system is proposed that includes nine (9) tubes having an "end-on" position parallel with the electron beam axis and a 12.7-cm (5-inch) center-to-center spacing. Two array configurations are being considered: a 3 x 3 array (See Figure 4-22) and a linear array, each centered with the electron beam axis. 


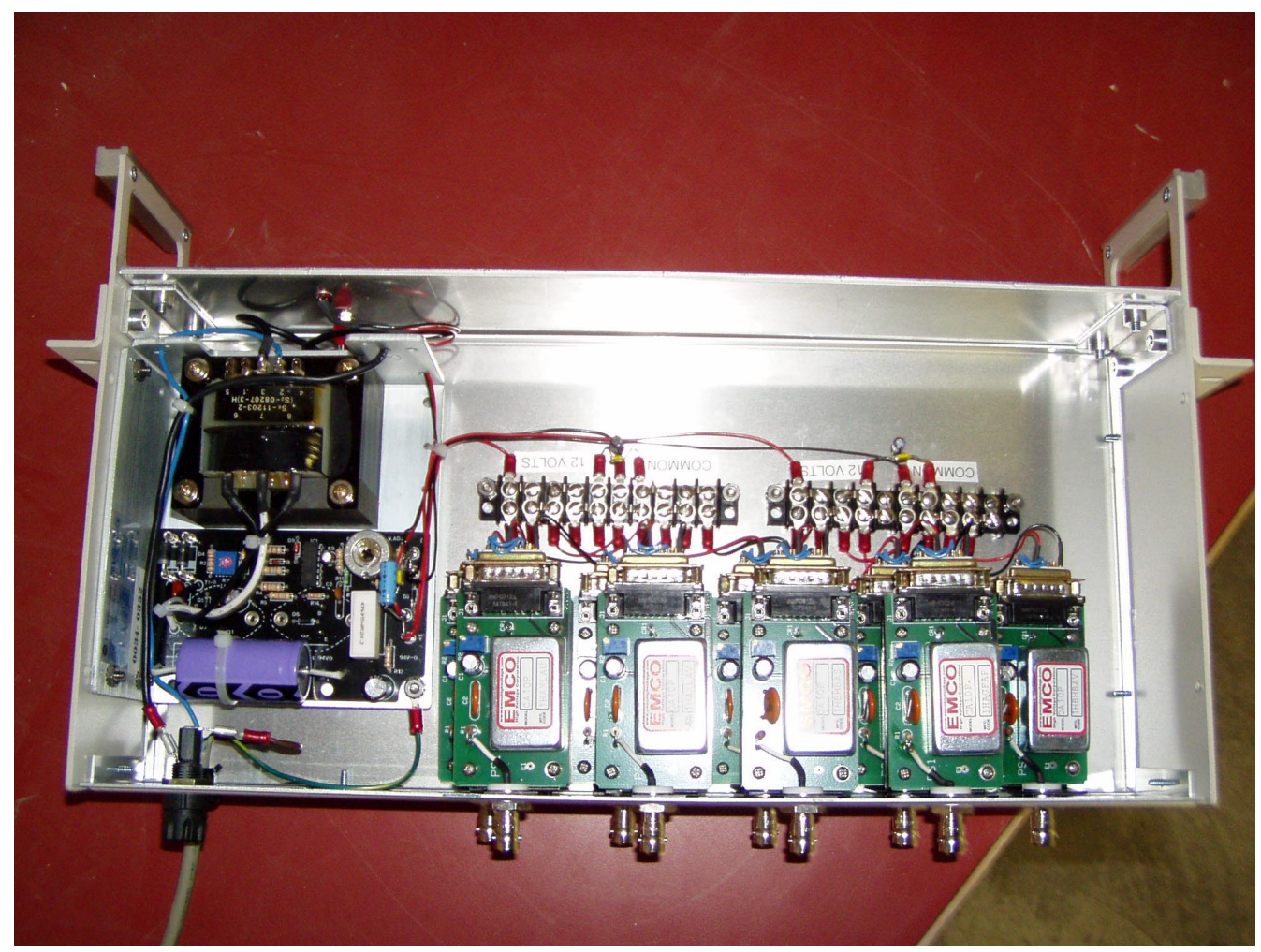

Figure 4-19. INL-built, high-voltage supply component for the grey-scale "imaging" system.

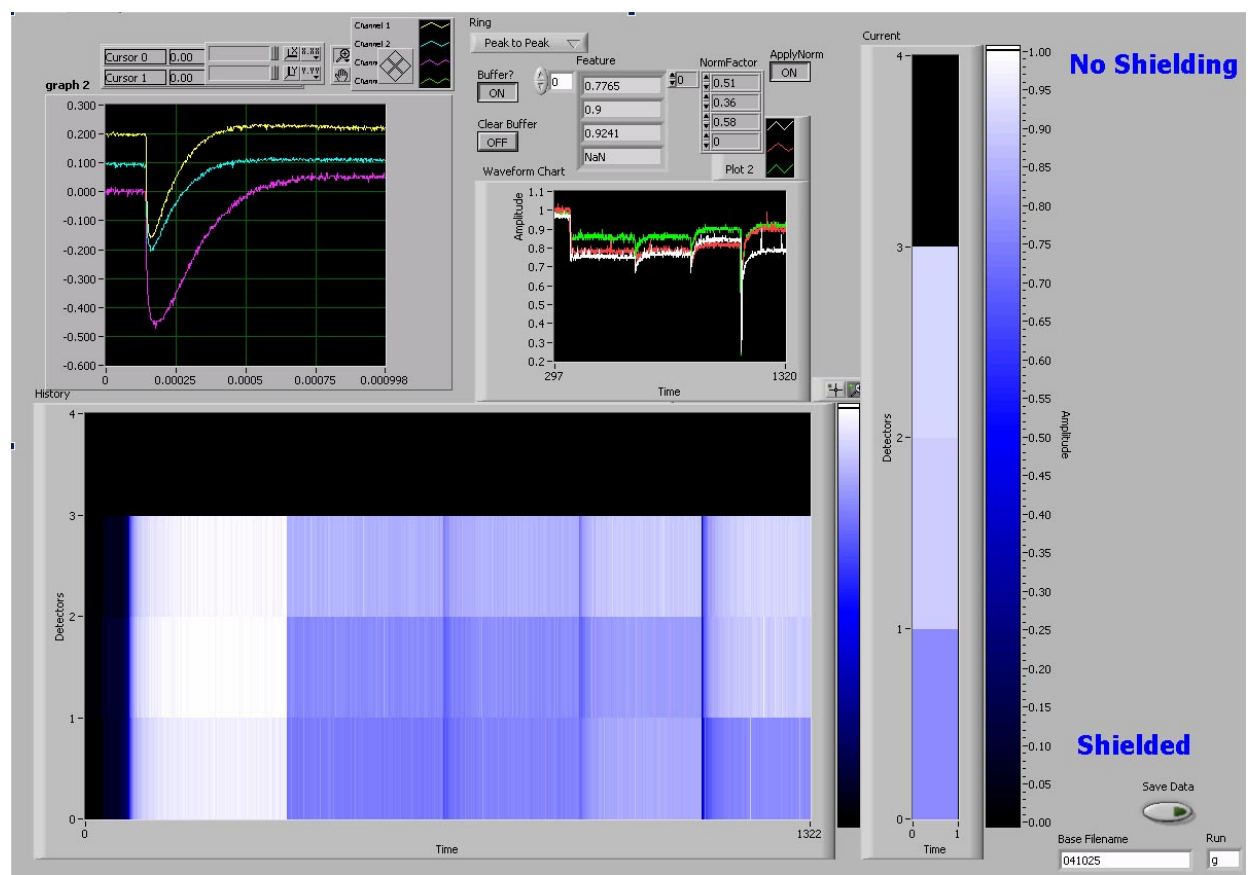

Figure 4-20. Labview-type developmental display for low-Z/high-Z "imaging” system development. 


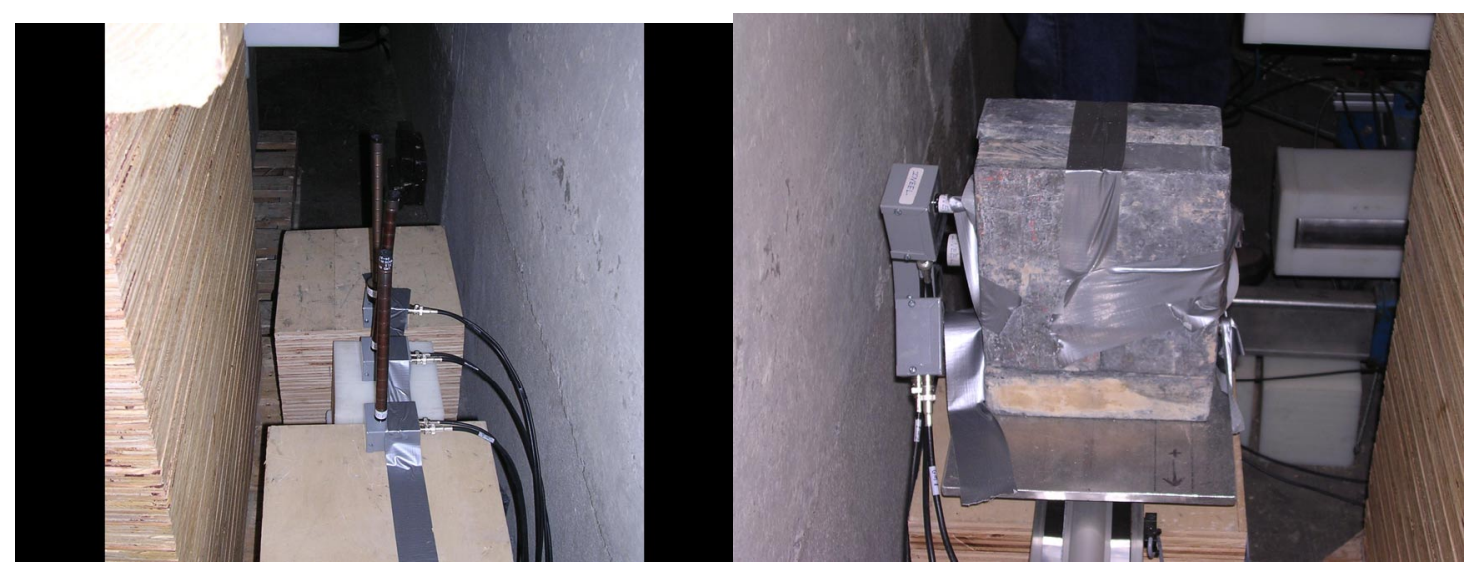

Figure 4-21. Three shielded and unshielded "imaging" detectors near a Plywood Calibration Pallet positioned vertically (left) and horizontally with lead (right).

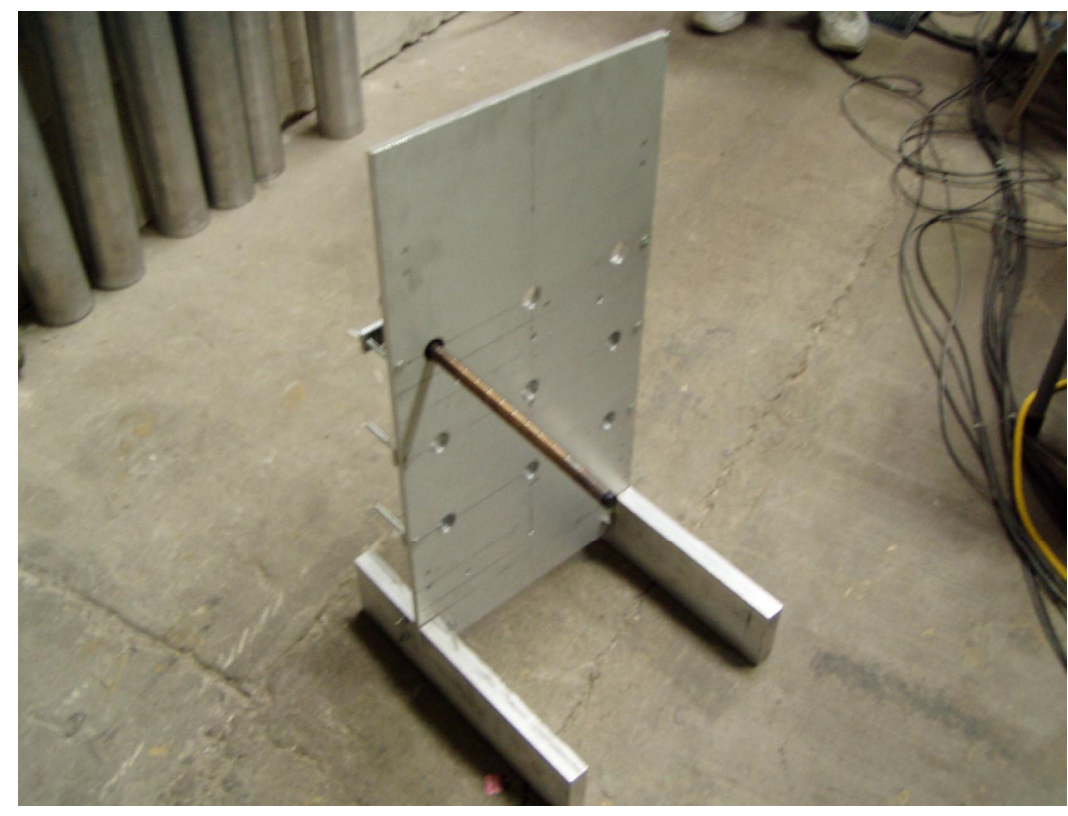

Figure 4-22. Prototypical "imaging" detector array configuration. 


\section{CALIBRATION PALLETS}

To help characterize the overall performance of the PPA system, as well as any other inspection system, a set of pallets have been fabricated and are referred to as Calibration Pallets. This assembly of pallets, schematically presented in Figure 5-1 and highlighted in Table 5-2 (with support components in Table 5-1), are not intended to represent an all inclusive set of cargo container loading configurations, but rather, represent a well defined set of radiation shielding configurations from which nuclear smuggling assessments can be performed for any given inspection technology. Each pallet is rectangular in shape, has a void designed in the center for nuclear material placement, has been sized to enable conventional side-by-side, double-stacked configurations, and allows maximum cargo loading flexibility. For example, the $1.07(\mathrm{~L}) \times 0.86(\mathrm{~W}) \times 1.02 \mathrm{~m}(\mathrm{D})$, Celotex Pallet is shown in Figure 5-2. Note the removable side plug to gain access to a center void of the shield material. These pallet designs can be transported from site to site and can support detailed numerical and experimental validation assessments. While these pallet designs originated from discussions within the DHS Active Interrogation Study Group, it is clear that other well-defined configurations can, and need to, be established such as a pallet of water. Detailed pallet descriptions are presented in Appendices B thru H.

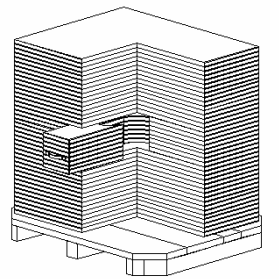

CELOTEX PALLET

$(\mathrm{L} \times W \times \mathrm{H}) 42^{\prime \prime} \times 34^{\prime \prime} \times 40^{n}$

$107 \mathrm{~cm} \times 86 \mathrm{~cm} \times 102 \mathrm{~cm}$
$197 \mathrm{bs} / 89.4 \mathrm{~kg}$

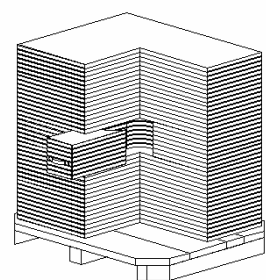

PLYWOOD PALLET

$(\mathrm{LW} \times \mathrm{WH}) 42^{2} \times 34^{\prime \prime} \times 40^{\prime \prime}$
$107 \mathrm{~cm} \times 86 \mathrm{~cm} \times 102 \mathrm{~m}$ $107 \mathrm{~cm} \times 86 \mathrm{~cm}$
$1060 \mathrm{lbs} / 481 \mathrm{~kg}$

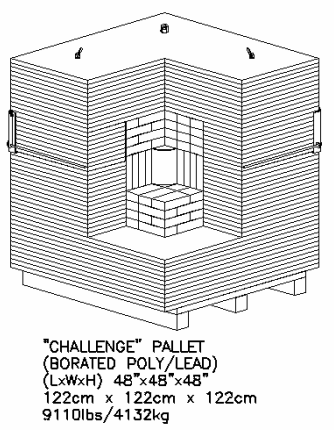

Calibration Pallet Configurations chamber for placing test materials.
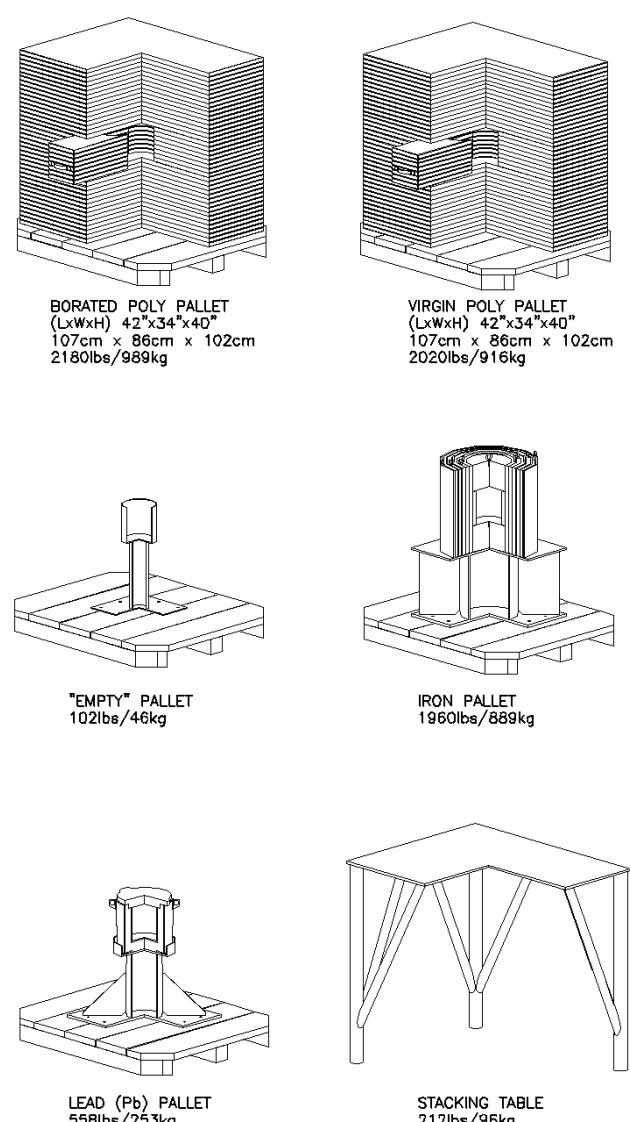

STACKING TABLE $2131 \mathrm{bs} / 96 \mathrm{~kg}$

Figure 5-1. Schematic of Calibration Pallets and the stacking table. 


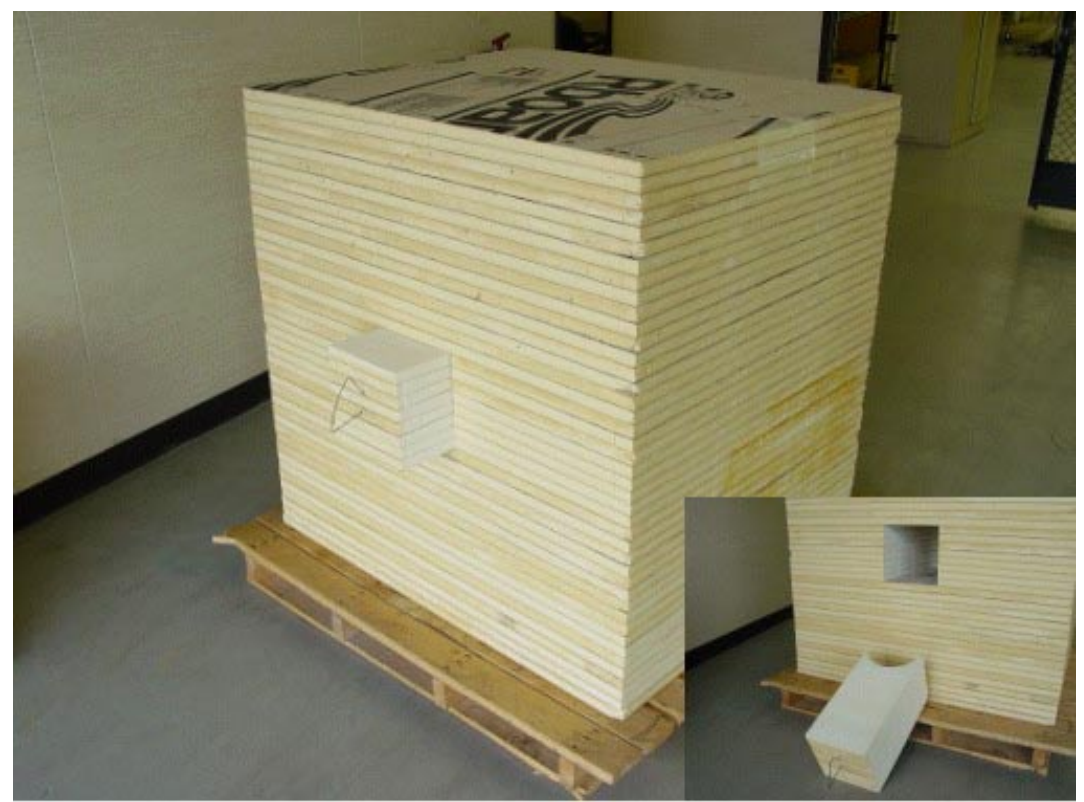

Figure 5-2 Celotex Calibration Pallet. (Note the removable side access plug.)

Table 5-1. Calibration pallet support components.

\begin{tabular}{lllcc}
$\begin{array}{c}\text { Component } \\
\text { Description }\end{array}$ & $\begin{array}{c}\text { Total Mass } \\
(\mathrm{lbm} / \mathrm{kg})\end{array}$ & Primary Material Type & $\begin{array}{c}\text { Primary Material } \\
\text { Density } \\
(\mathrm{g} / \mathrm{cc})\end{array}$ & $\begin{array}{c}\text { Appendix } \\
\text { Description }\end{array}$ \\
\hline Base & $85 / 38$ & Wood (Pine) & 0.39 & $\mathrm{~B}$ \\
Stacking Frame & $212 / 96$ & Aluminum & 2.70 & $\mathrm{C}$ \\
\hline
\end{tabular}

Table 5-2. Calibration Pallet designs.

\begin{tabular}{|c|c|c|c|c|}
\hline $\begin{array}{c}\text { Pallet } \\
\text { Description }\end{array}$ & $\begin{array}{l}\text { Total Mass } \\
(1 \mathrm{bm} / \mathrm{kg})\end{array}$ & $\begin{array}{c}\text { Primary Material } \\
\text { Type }\end{array}$ & $\begin{array}{c}\text { Primary Material } \\
\text { Density } \\
(\mathrm{g} / \mathrm{cc})\end{array}$ & $\begin{array}{c}\text { Appendix } \\
\text { Description }\end{array}$ \\
\hline 1) Empty & $102 / 46$ & Aluminum & 2.70 & $\mathrm{D}$ \\
\hline 2) Celotex & $197 / 90$ & "Celotex" & 0.05 & $\mathrm{E}$ \\
\hline 3) Wood & $1060 / 482$ & Plywood & 0.48 & $\mathrm{E}$ \\
\hline 4) Polyethylene & $2031 / 923$ & Polyethylene & 0.95 & E \\
\hline $\begin{array}{l}\text { 5) Borated olyethylene } \\
\text { (B-poly) }\end{array}$ & $2229 / 1012$ & $\begin{array}{l}5 \% \text {-Borated } \\
\text { Polyethylene }\end{array}$ & 1.05 & $\mathrm{E}$ \\
\hline 6) Lead & $558 / 254$ & Lead & 10.8 & $\mathrm{~F}$ \\
\hline 7) Iron & $1960 / 890$ & $\mathrm{Fe}$ & 7.80 & G \\
\hline 8) Lead/B-Poly “Challenge" & $9110 / 4136$ & $\begin{array}{l}\text { 5\%-Borated Poly./ } \\
\text { Lead }\end{array}$ & $\begin{array}{l}1.05 \\
10.8 \\
\end{array}$ & $\mathrm{H}$ \\
\hline
\end{tabular}




\section{SYSTEM AND COMPONENT ANALYSES}

The following numerical and experimental analyses were performed to characterize the capabilities and performance of the PPA inspection system.

\subsection{DU Validation Assessment}

Two separate experimental benchmark configurations were developed for validating the numerical calculations. Comparisons between experimental and numerical values provide a valuable benchmark or validation as to how good the numerical calculations can predict an actual experiment. Biases identified through the comparison process can also help reveal sensitivities associated with the different materials. Perhaps the most important reason for the benchmarking is to give credence to the numerical simulations involving parametric changes.

The first benchmark involved the bremstrahlung irradiation of depleted uranium (DU) plates in air and experimentally measuring the delayed neutron signal response in an array of vertically stacked PPNDs. The second, more complex, shielded benchmark used the same general experimental configuration but included a multi-pallet configuration with the DU plates hidden and centered inside the Plywood Calibration Pallet (see Appendix E). The DU position in both benchmark cases was identical. The latter experimental configuration is shown in Figure 6-1.

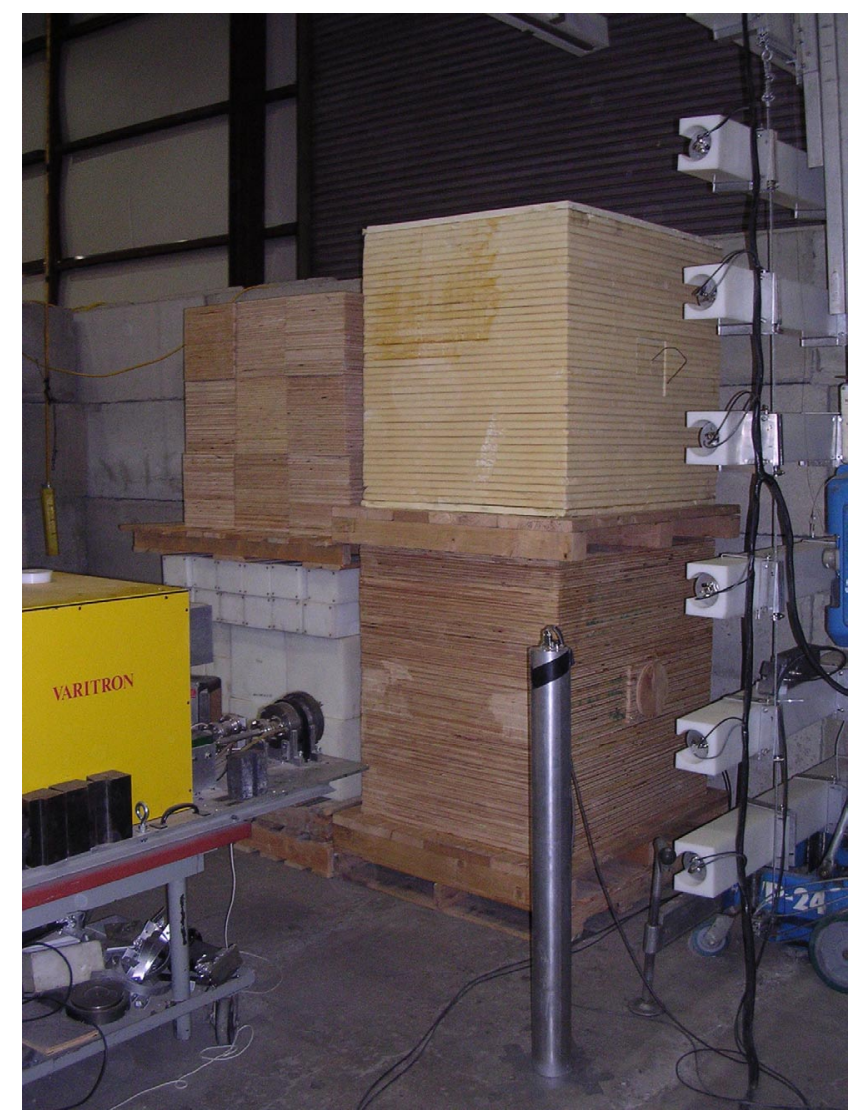

Figure 6-1. Multi-pallet configuration showing four pallets, the PPND detector array (right), and the VARITRON accelerator (yellow box, lower left) with a tungsten collimator. 
This benchmark configuration included a $2 \times 2$ array of four pallets (two plywood, one Celotex, and one polyethylene). The two-pallet stack nearest the detectors as composed of the Plywood Calibration Pallet on the bottom (lower right) and the Celotex Calibration Pallet on the top. The plywood pallet was positioned such that the accelerator beamline was centered on its front face that is $78 \mathrm{~cm}$ from the photon source. This plywood pallet contained the DU plates when nuclear material was present. The nearest shroud of the planar array of PPND detectors was positioned $53 \mathrm{~cm}$ from the surface of the Plywood Calibration Pallet. The other dual pallet stack (to the left of the calibration pallet stack) is built-up with polyethylene on the bottom and plywood on top.

The accelerator was operated with a nominal $10 \mathrm{-MeV}$ electron beam energy, had a pulsing rate of $125 \mathrm{~Hz}$ (i.e., $8 \mathrm{~ms}$ between accelerator pulses), and had the photon source positioned at about $132 \mathrm{~cm}$ from the center of the DU plates. The DU plates were oriented at a 45-degree angle to the beam axis. The four approximately 14-cm square, rectangular pieces of DU were used as representative nuclear material and were modeled as a single rectangular block having a combined plate dimensions of $12.7 \times 15.24 \times 1.331 \mathrm{~cm}$ (thick) and resulting in a total volume of $257.6 \mathrm{cc}$ with a mass of $4.9 \mathrm{~kg}$. The actual mass of all four DU plates, $4.8 \mathrm{~kg}$, is slightly less than the numerical modeled value because of a series of small mounting holes in the DU plates that were not specifically modeled. Finally, a unshrouded, "He-3 tube-only" PPND-type detector (shown in the lower center of Figure 6-1) was used to monitor the overall time-dependent thermal neutron responses.

\subsubsection{Numerical Calculation Methodology}

The calculation methodology was based on the MCNPX version $2.5 \mathrm{e}$ (beta version) ${ }^{10}$ computer code to perform electron-photon-neutron transport calculations. The MCNP models simulated the experimental setups in explicit detail. This included the accelerator target and collimator, the plywood, Celotex, and polyethylene (poly) pallets, the array of PPNDs, and the concrete floor and shield walls surrounding the experimental setup.

Due to unsuccessful attempts at developing a viable single MCNPX model for these benchmark scenarios, two transport calculations were utilized. The first transport calculation estimates the fission rates in the depleted uranium (or highly enriched uranium, if applicable) concealed in the plywood pallet by first transporting accelerated electrons into a tungsten converter to generate the bremstrahlung photons. These photons are then transported through the air and/or pallets into nuclear material. Included in these transport calculation are: (1) photoneutrons produced in the air and pallet materials, (2) photofission and neutron-induced fission neutrons in the nuclear material, (3) delayed neutrons in the nuclear material, (4) prompt fission gammas and neutrons produced by each fission event, and (5) radiative capture gammas produced by the thermal neutrons. The second transport calculation involved transport of the induced prompt and delayed neutrons emanating out of the nuclear material through the air and/or the Plywood Calibration Pallet and into the PPND detectors.

\subsubsection{Results Comparison}

The experimental and numerical model predictions for the total detector counts for both the benchmark cases are given in Table 6-1. The neutron detector response chosen for these benchmark comparisons is the second PPND detector up from the ground in the vertical stack of PPND detectors (See Figure 6-1). This detector is approximately at the same elevation as the DU and, naturally, received the greatest number of delayed neutron counts relative to the other PPND detectors. The detector responses, both experimental and numerical, are for a total 120 -seconds acquisition times using a delayed neutron window from approximately 2 to 8 milliseconds after each accelerator pulse. 
Table 6-1. Comparison of experimental and numerical benchmark results.

\begin{tabular}{|c|c|c|c|c|}
\hline Benchmark & $\begin{array}{l}\text { Nominal Electron } \\
\text { Energy } \\
(\mathrm{MeV}) \\
\end{array}$ & $\begin{array}{l}\text { Experimental } \\
\text { Detector Response } \\
(\text { Net counts }) \\
\end{array}$ & $\begin{array}{l}\text { Numerical } \\
\text { Detector Response } \\
\text { (Total counts) } \\
\end{array}$ & E/C Ratio \\
\hline DU in air & 10 & $13,348^{b}$ & $9,368^{\mathrm{c}}$ & 1.42 \\
\hline DU in plywood & 10 & $441^{\mathrm{d}}$ & $415^{\mathrm{c}}$ & 1.06 \\
\hline \multicolumn{5}{|c|}{ 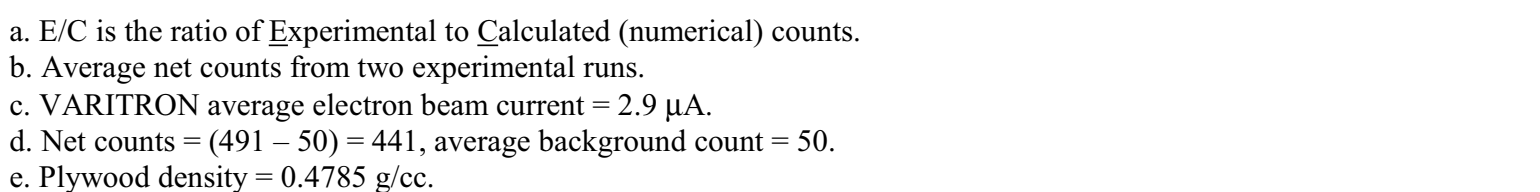 } \\
\hline
\end{tabular}

The calculated delayed neutron total counts are in very good agreement with the experimental values, despite the complex multi-step calculational methodology, uncertainties in the delayed neutron yields (photofissions and induced neutron fissions), delayed neutron spectra, photoneutron and photofission cross section data, and measured input data. The INL photonuclear team is continuing to improve and verify its modeling capabilities with additional experimental validation and coordination with LANL MCNPX developers.

\subsection{Receiver-Operator-Characteristic (ROC) Curves}

Receiver-Operator-Characteristic (ROC) Curves have been developed to help characterize the performance of any inspection technology. Among other capabilities, these curves identify the probability of detection (i.e., true positive) versus the probability of false positive detection based on a series of experimental tests. The basis of a ROC curves uses the probability distributions of measured responses with and without nuclear material, such as schematically presented in Figure 6-2. The decision point (vertical dashed line) is based on the confidence of detection and represents the area under the probability distribution curve $\left(\mathrm{p}_{1}(\mathrm{y})\right)$ greater than the decision point (e.g., region $\mathrm{R}_{1}$ of $\mathrm{p}_{1}(\mathrm{y})$ ). The overlap of the background probability distribution $\left(\mathrm{p}_{\mathrm{o}}(\mathrm{y})\right)$ greater than the decision point represents the probability of a false positive. INL

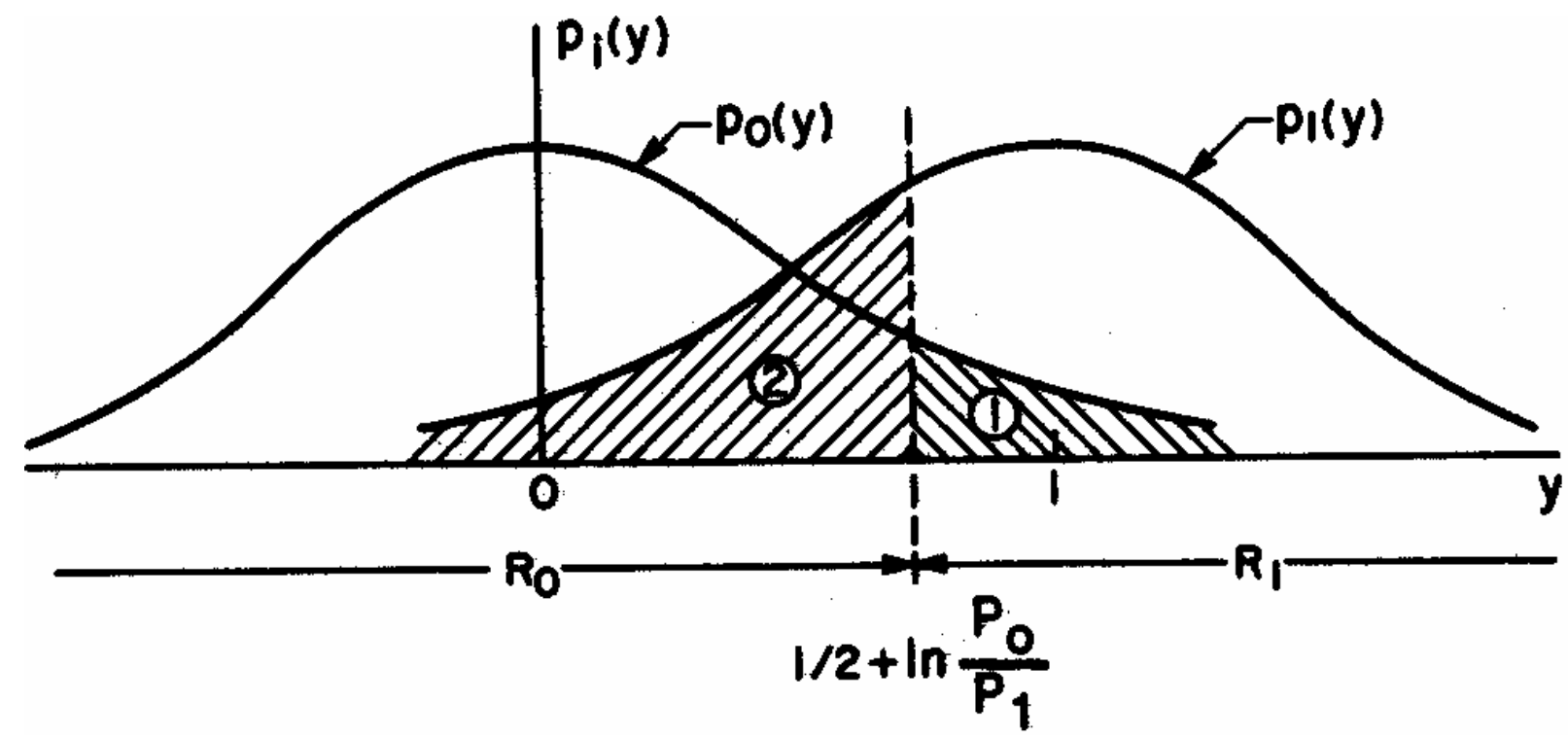

Figure 6-2. Typical probability distributions for ROC curve development. 
develops its ROC curves using repeated measurements with and without nuclear materials in a given cargo loading configuration. The measurements are developed into ROC curves using a commercially available, statistical software package called "Analyse-it." (Version 1.71 by Analyse-it Software, Ltd.).

Multiple test data have been compiled and plotted using the ROC method at a 99.9-percent confidence level for the DU validation tests described in the previous section (i.e., with and without multiple pallets). The actual Analyse-it software output display is shown in Figure 6-3 for this case. Note, the resulting "ideal" detection performance provided by PPND detector nearest the DU.
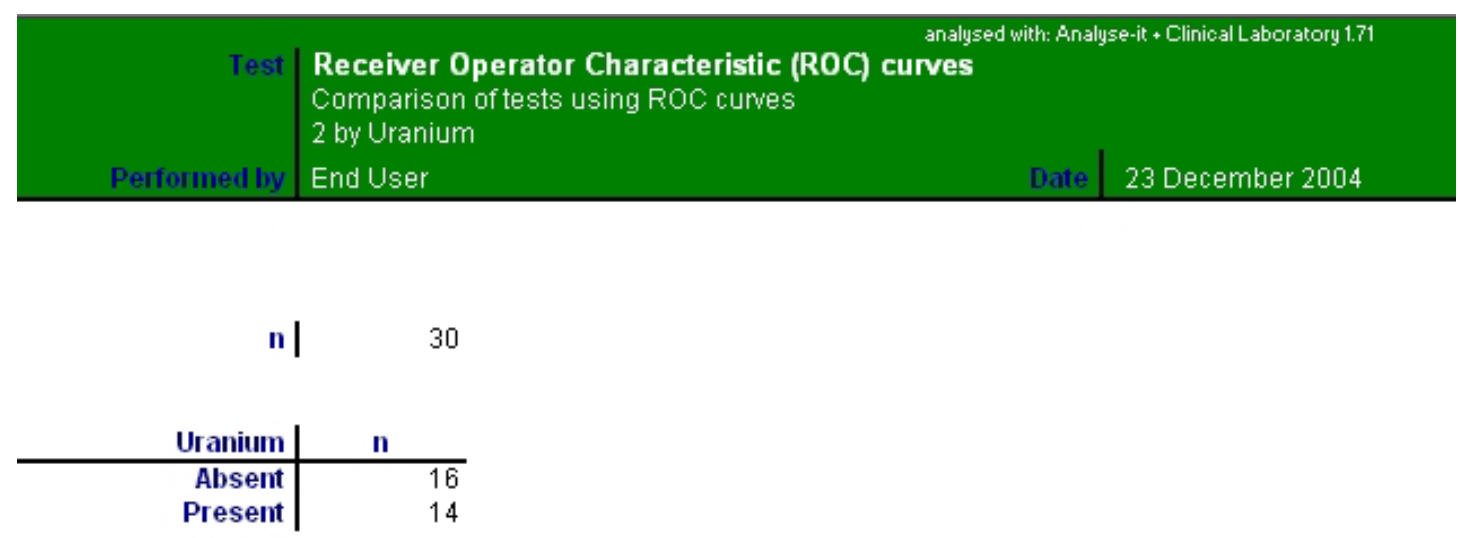

\begin{tabular}{r|r:r:r:r:r} 
Curve & Area & SE & $\mathbf{p}$ & $\mathbf{9 9 . 9} \% \mathrm{Cl}$ of Area & Uranium = Present \\
\hline 2 & 1.000 & 0.0000 & 1.0000 & 1.000 to 1.000 & have highervalues
\end{tabular}

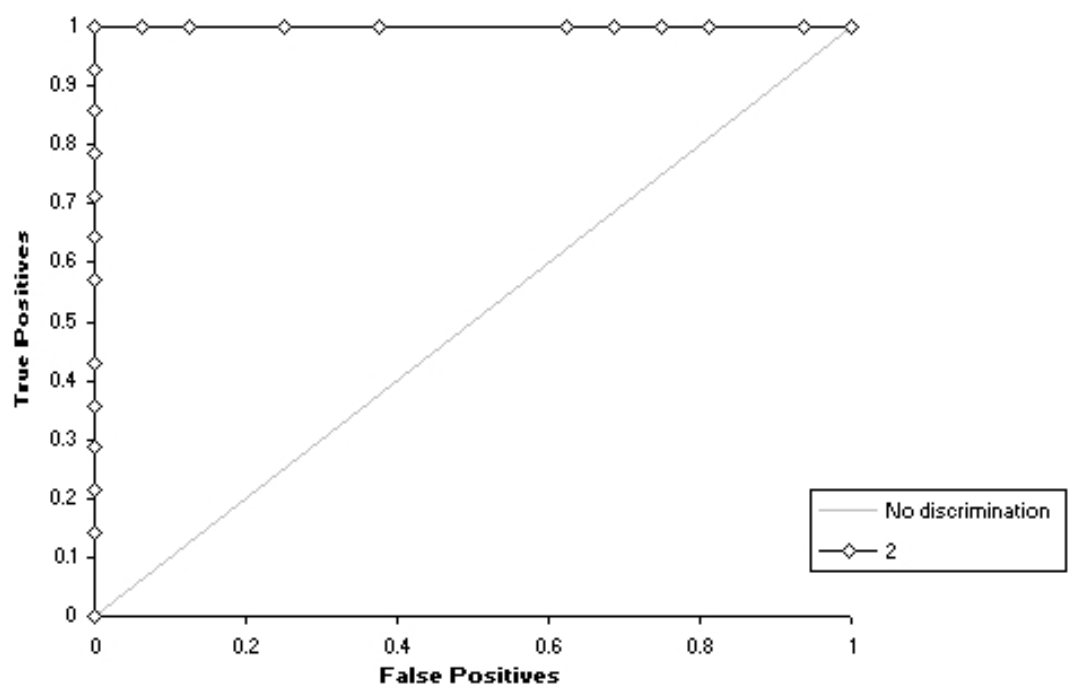

Figure 6-3. Analyse-it output (i.e., ROC) for DU validation testing. 
Another experiment was conducted to assess the ROC performance of each PPND on the mobile detector platform using only a Plywood and Celotex Calibration Pallet configuration as shown in Figure 6-4. Note, the PPND Location \# 2 is nearest to the center of the plywood pallet where the uranium (when included) is located. This location provides the highest counts when the uranium is present and, therefore, is an important detector location for the overall detection process for this given configuration. While only individual detectors have been considered in this assessment, increased detection sensitivity can be obtained by evaluating groups of detectors. The ROC curves for several detector locations are shown in Figure 6-5 for a series of 120 -s acquisitions and using a nominal $10-\mathrm{MeV}$ interrogation. Note, only one detector (or detector group) is required for any given inspection to provide positive detection. Even at the 99\% confidence interval, the ROC shows that PPND at Location \#2 has a 100\% detection capability with $0 \%$ false alarm rate! Note, while several detector positions provided 100-percent detection probability, the detector location with the most challenging opportunity in detecting this nuclear material with this loading configuration is readily identified as detector Location \#7.

\subsection{Electron Beam Energy Assessments}

To assess the optimal performance of the PPA inspection system a set of numerical and experimental assessments were performed.
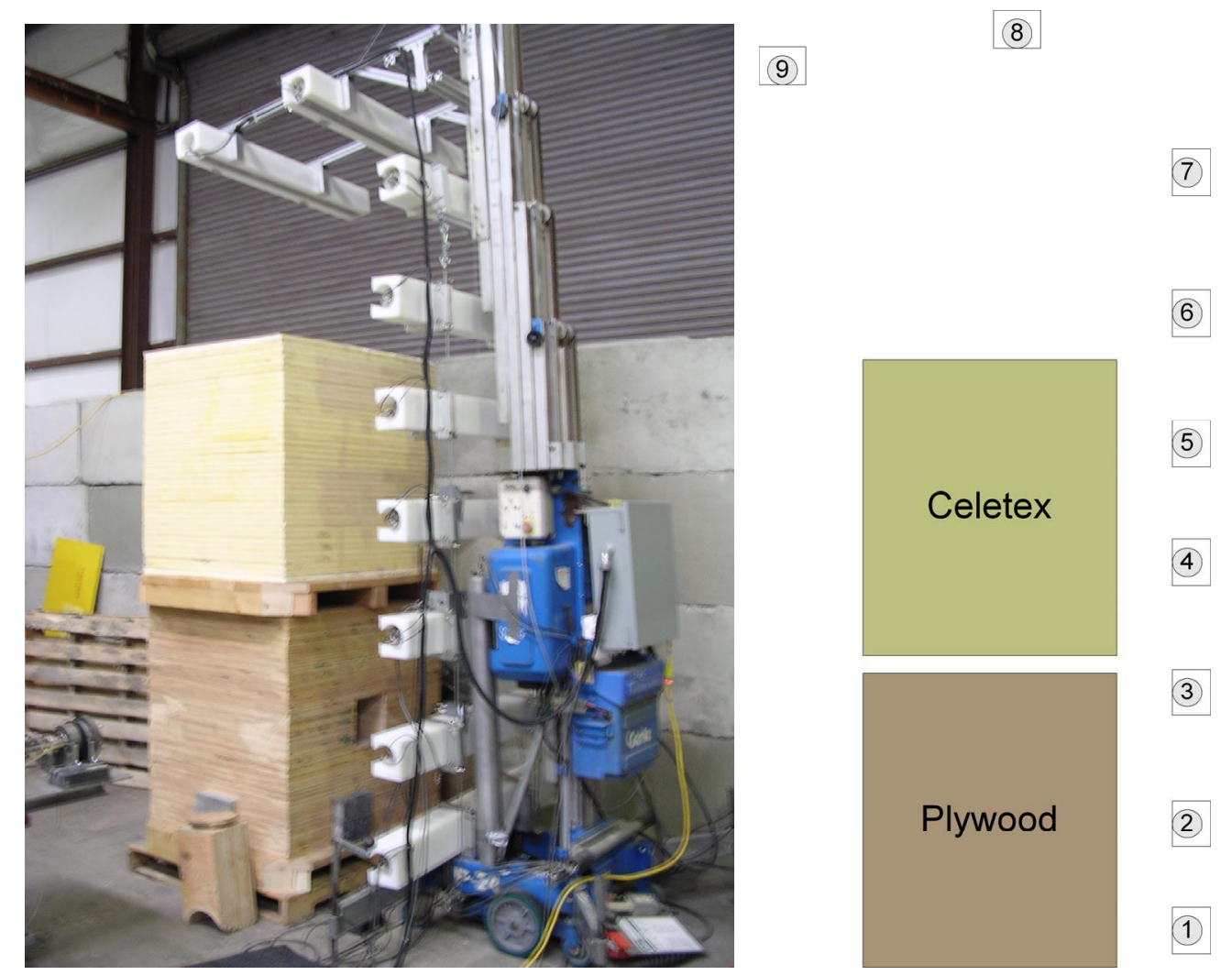

Figure 6-4. Actual test configuration (left) with diagram of test setup and identified detector locations. 


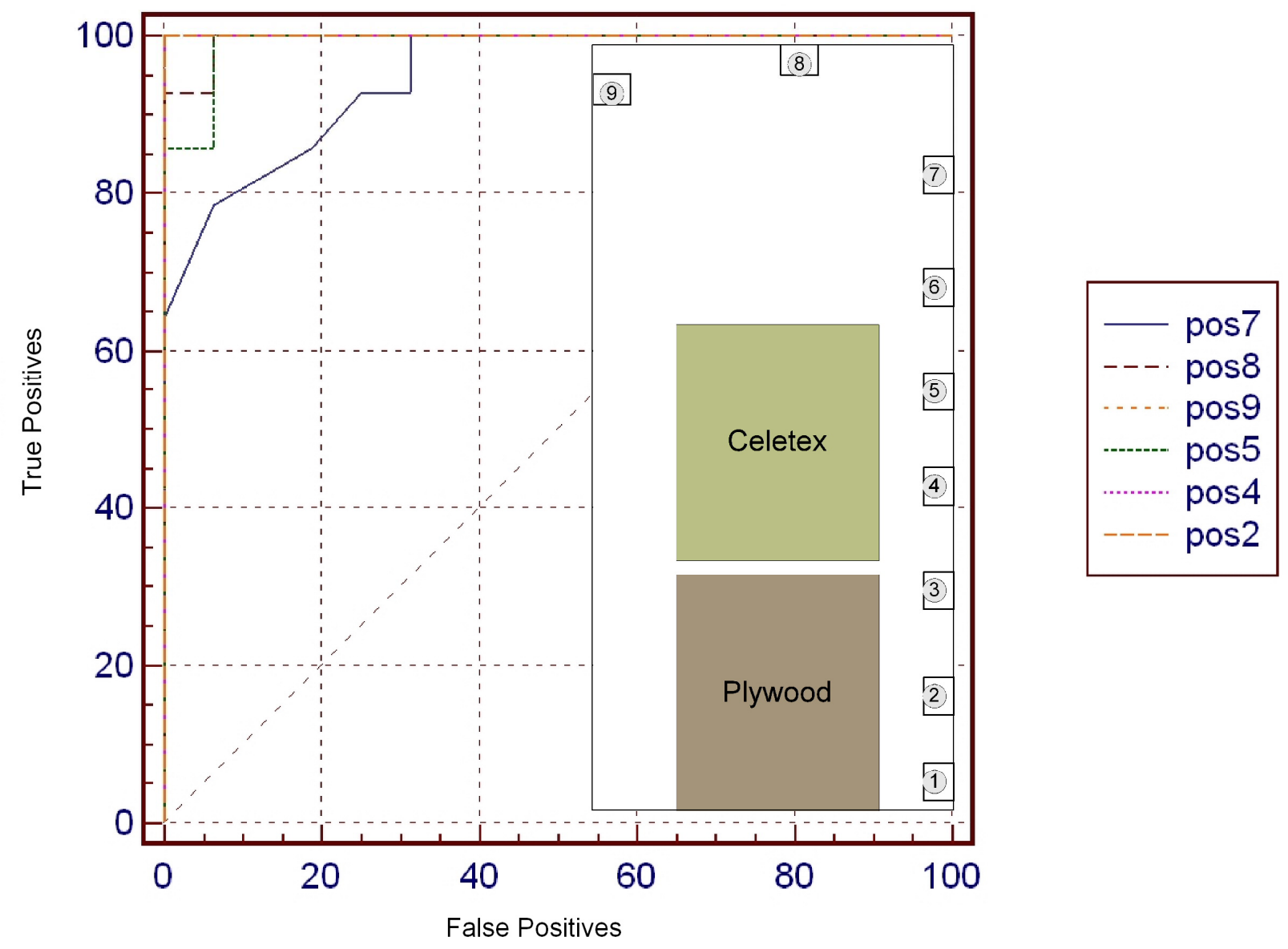

Figure 6-5. ROC curves for various PPND locations.

\subsubsection{Numerical Evaluations}

The MCNPX numerical models and methodologies discussed previously were used to calculate the fissions per source electron (fiss/se) for three different parametric studies. The goal of these studies was to help identify an optimal electron beam energy that would more efficiently fission a shielded DU/HEU object and thereby increase the total delayed neutron counts in a PPND detector. The three objects used in the three parametric studies were: (1) the INL DU plates, (2) a hypothetical HEU plate with the physical dimension of the INL DU plates, and (3) an HEU sphere.

6.3.1.1 DU Plates. The first parametric studied involves the INL DU plates, modeled as a single combined DU plate, in the Plywood Calibration Pallet (as in Section 6.1) as a function of electron beam energy. Using the methodology described in Section 6.1.1, the predicted results are presented in Table 6-2 and plotted in Figure 6-6. For comparison purposes, the four fission components, namely U-238 and U-235 photofission and neutron-induced fission components, are specified separately. In addition, the last column identifies the total fissions in the DU per source electron on the converter. Notice the yield increase of nearly 350 between the 8 - and $20-\mathrm{MeV}$ operations. In all cases the neutron yield is dominated by the photofissioning of DU and the yield while continuing to increase with electron beam energy does so at a decreasing rate. 
Table 6-2. Fission components for the DU plates in the Plywood Pallet.

\begin{tabular}{cccccc}
\hline $\begin{array}{c}\text { Electron } \\
\begin{array}{c}\text { Energy } \\
(\mathrm{MeV})\end{array}\end{array}$ & $\begin{array}{c}\text { U-238 } \\
\text { Photofission } \\
\text { (fiss/se) }\end{array}$ & $\begin{array}{c}\text { U-235 } \\
\text { Photofission } \\
\text { (fiss/se) }\end{array}$ & $\begin{array}{c}\text { U-238 } \\
\text { Neutron-induced } \\
\text { fission } \\
\text { (fiss/se) }\end{array}$ & $\begin{array}{c}\text { U-235 } \\
\text { Neutron-induced } \\
\text { fission } \\
\text { (fiss/se) }\end{array}$ & $\begin{array}{c}\text { TOTAL } \\
\text { Fissions } \\
\text { (fiss/se) }\end{array}$ \\
\hline 6 & $9.05 \mathrm{E}-10$ & $8.31 \mathrm{E}-13$ & $5.66 \mathrm{E}-11$ & $4.35 \mathrm{E}-11$ & $1.01 \mathrm{E}-09$ \\
8 & $3.54 \mathrm{E}-08$ & $1.10 \mathrm{E}-10$ & $2.34 \mathrm{E}-09$ & $2.23 \mathrm{E}-09$ & $4.01 \mathrm{E}-08$ \\
10 & $2.22 \mathrm{E}-07$ & $8.45 \mathrm{E}-10$ & $1.79 \mathrm{E}-08$ & $1.63 \mathrm{E}-08$ & $2.57 \mathrm{E}-07$ \\
12 & $9.34 \mathrm{E}-07$ & $4.05 \mathrm{E}-09$ & $8.99 \mathrm{E}-08$ & $6.95 \mathrm{E}-08$ & $1.10 \mathrm{E}-06$ \\
14 & $2.40 \mathrm{E}-06$ & $1.17 \mathrm{E}-08$ & $2.51 \mathrm{E}-07$ & $1.89 \mathrm{E}-07$ & $2.85 \mathrm{E}-06$ \\
16 & $4.90 \mathrm{E}-06$ & $2.44 \mathrm{E}-08$ & $5.37 \mathrm{E}-07$ & $4.02 \mathrm{E}-07$ & $5.86 \mathrm{E}-06$ \\
18 & $8.02 \mathrm{E}-06$ & $3.94 \mathrm{E}-08$ & $8.92 \mathrm{E}-07$ & $6.63 \mathrm{E}-07$ & $9.61 \mathrm{E}-06$ \\
20 & $1.16 \mathrm{E}-05$ & $5.61 \mathrm{E}-08$ & $1.29 \mathrm{E}-06$ & $9.34 \mathrm{E}-07$ & $1.39 \mathrm{E}-05$ \\
\hline
\end{tabular}

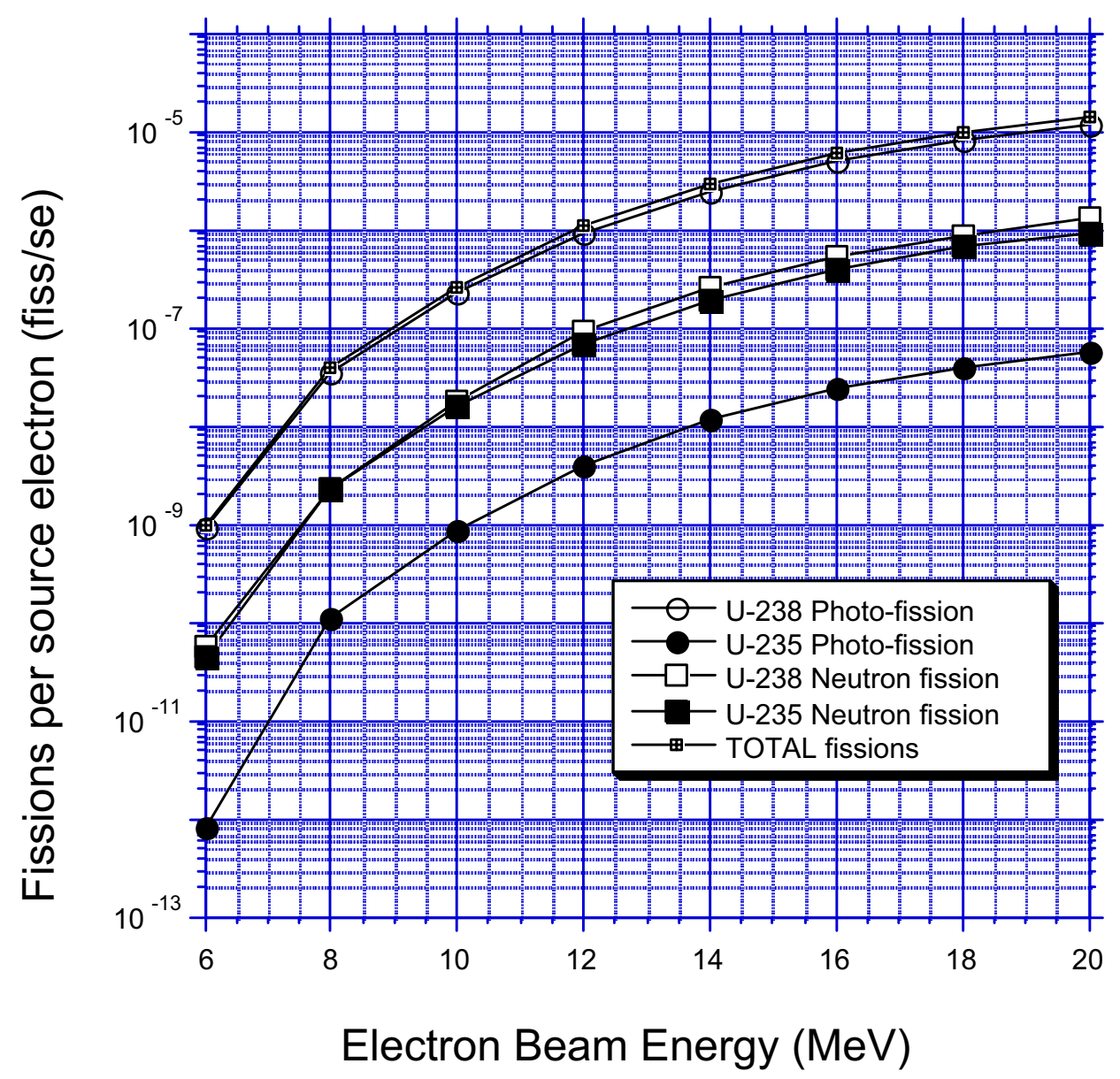

Figure 6-6. Photofission and neutron-induced fission components as a function of electron beam energy for the DU plates in the Plywood Calibration Pallet. 
6.3.1.2 HEU PLATES. In the second parametric study, the DU plates in the preceding section were replaced with HEU plates (93.15 wt\% U-235 enrichment). The HEU metal density was assumed to be $18.7 \mathrm{~g} / \mathrm{cc}$, or slightly lower than the assumed DU density of $19.1 \mathrm{~g} / \mathrm{cc}$. The fissions per source electron are presented in Table 6-3 and plotted on Figure 6-7.

Table 6-3. Fission components for the HEU plates in the Plywood Pallet.

\begin{tabular}{cccccc}
\hline $\begin{array}{c}\text { Electron } \\
\begin{array}{c}\text { Energy } \\
(\mathrm{MeV})\end{array}\end{array}$ & $\begin{array}{c}\text { U-238 } \\
\text { Photofission } \\
\text { (fiss/se) }\end{array}$ & $\begin{array}{c}\text { U-235 } \\
\text { Photofission } \\
\text { (fiss/se) }\end{array}$ & $\begin{array}{c}\text { Neutron-induced } \\
\text { fission } \\
\text { (fiss/se) }\end{array}$ & $\begin{array}{c}\text { U-235 } \\
\text { Neutron-induced } \\
\text { fission } \\
\text { (fiss/se) }\end{array}$ & $\begin{array}{c}\text { TOTAL } \\
\text { Fissions } \\
\text { (fiss/se) }\end{array}$ \\
\hline 6 & $6.32 \mathrm{E}-11$ & $3.02 \mathrm{E}-10$ & $5.06 \mathrm{E}-12$ & $1.12 \mathrm{E}-09$ & $1.49 \mathrm{E}-09$ \\
8 & $2.40 \mathrm{E}-09$ & $4.06 \mathrm{E}-08$ & $3.21 \mathrm{E}-10$ & $4.57 \mathrm{E}-08$ & $8.89 \mathrm{E}-08$ \\
10 & $1.49 \mathrm{E}-08$ & $3.07 \mathrm{E}-07$ & $2.63 \mathrm{E}-09$ & $3.36 \mathrm{E}-07$ & $6.60 \mathrm{E}-07$ \\
12 & $6.26 \mathrm{E}-08$ & $1.47 \mathrm{E}-06$ & $1.36 \mathrm{E}-08$ & $1.66 \mathrm{E}-06$ & $3.20 \mathrm{E}-06$ \\
14 & $1.62 \mathrm{E}-07$ & $4.29 \mathrm{E}-06$ & $4.00 \mathrm{E}-08$ & $4.69 \mathrm{E}-06$ & $9.18 \mathrm{E}-06$ \\
16 & $3.31 \mathrm{E}-07$ & $8.94 \mathrm{E}-06$ & $8.45 \mathrm{E}-08$ & $9.79 \mathrm{E}-06$ & $1.91 \mathrm{E}-05$ \\
18 & $5.42 \mathrm{E}-07$ & $1.45 \mathrm{E}-05$ & $1.37 \mathrm{E}-07$ & $1.57 \mathrm{E}-05$ & $3.09 \mathrm{E}-05$ \\
20 & $7.79 \mathrm{E}-07$ & $2.05 \mathrm{E}-05$ & $1.96 \mathrm{E}-07$ & $2.23 \mathrm{E}-05$ & $4.37 \mathrm{E}-05$ \\
\hline
\end{tabular}

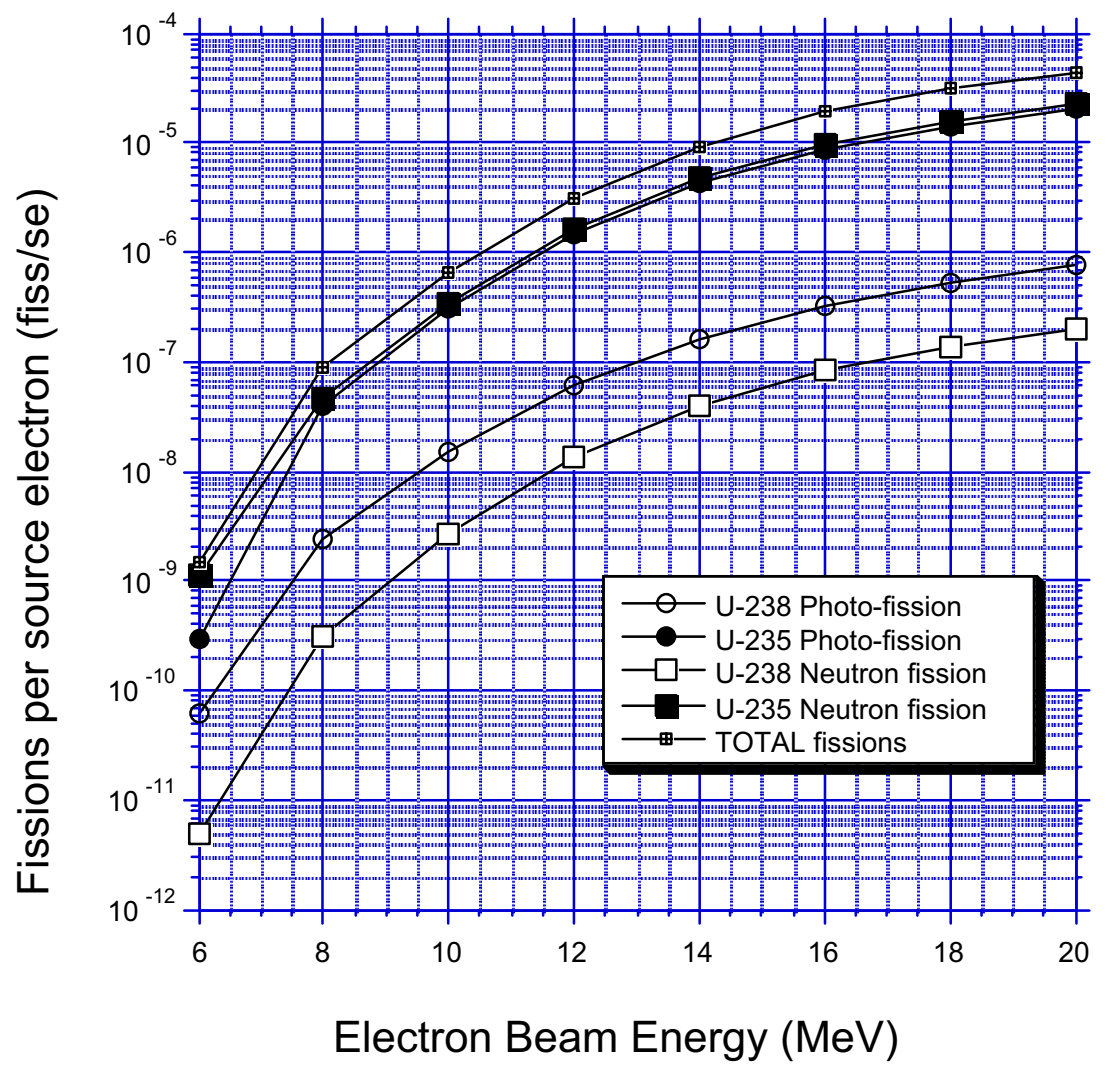

Figure 6-7 Photofission and neutron-induced fission components as a function of electron beam energy for the HEU plates in the Plywood Calibration Pallet. 
As with DU, the yields increase with increasing electron beam energy. Comparing the data in Tables 6-2 with 6-3, we find that the total fission rate is increased by a factor of approximately three for the HEU material case. This is believed to be due to both the higher U-235 photofission cross section (twice that of U-238) and the large thermal neutron fission cross section of U-235 relative to U-238.

6.3.1.3 HEU Sphere. To study geometry effects, a third parametric study involved replacing the HEU plates in the plywood with a 5-kg HEU sphere. The HEU sphere had the following characteristics: $93.15 \mathrm{wt} \% \mathrm{U}-235$ enrichment, diameter of approximately $3.998 \mathrm{~cm}$, volume of $267.7 \mathrm{cc}$, and an assumed density of $18.7 \mathrm{~g} / \mathrm{cc}$. Predicted results from this third parametric study are presented in Table 6-4 and are plotted in Figure 6-8.

Table 6-4. Fission components for the HEU sphere in the Plywood Calibration Pallet.

\begin{tabular}{cccccc}
\hline $\begin{array}{c}\text { Electron } \\
\text { Energy } \\
(\mathrm{MeV})\end{array}$ & $\begin{array}{c}\text { U-238 } \\
\text { Photofission } \\
\text { (fiss/se) }\end{array}$ & $\begin{array}{c}\text { U-235 } \\
\text { Photofission } \\
\text { (fiss/se) }\end{array}$ & $\begin{array}{c}\text { U-238 } \\
\text { Nitron-induced } \\
\text { fission } \\
\text { (fiss/se) }\end{array}$ & $\begin{array}{c}\text { U-235 } \\
\text { Neutron-induced } \\
\text { fission } \\
\text { (fiss/se) }\end{array}$ & $\begin{array}{c}\text { TOTAL } \\
\text { Fissions } \\
\text { (fiss/se) }\end{array}$ \\
\hline 6 & $2.85 \mathrm{E}-11$ & $1.38 \mathrm{E}-10$ & $5.24 \mathrm{E}-12$ & $6.88 \mathrm{E}-10$ & $8.60 \mathrm{E}-10$ \\
8 & $1.05 \mathrm{E}-09$ & $1.78 \mathrm{E}-08$ & $2.87 \mathrm{E}-10$ & $3.12 \mathrm{E}-08$ & $5.03 \mathrm{E}-08$ \\
10 & $6.82 \mathrm{E}-09$ & $1.40 \mathrm{E}-07$ & $2.08 \mathrm{E}-09$ & $2.31 \mathrm{E}-07$ & $3.81 \mathrm{E}-07$ \\
12 & $2.86 \mathrm{E}-08$ & $6.71 \mathrm{E}-07$ & $1.19 \mathrm{E}-08$ & $1.05 \mathrm{E}-06$ & $1.77 \mathrm{E}-06$ \\
14 & $6.81 \mathrm{E}-08$ & $1.79 \mathrm{E}-06$ & $3.36 \mathrm{E}-08$ & $2.97 \mathrm{E}-06$ & $4.86 \mathrm{E}-06$ \\
16 & $1.43 \mathrm{E}-07$ & $3.85 \mathrm{E}-06$ & $6.82 \mathrm{E}-08$ & $6.30 \mathrm{E}-06$ & $1.04 \mathrm{E}-05$ \\
18 & $2.28 \mathrm{E}-07$ & $6.05 \mathrm{E}-06$ & $1.08 \mathrm{E}-07$ & $9.76 \mathrm{E}-06$ & $1.61 \mathrm{E}-05$ \\
20 & $3.30 \mathrm{E}-07$ & $8.63 \mathrm{E}-06$ & $1.60 \mathrm{E}-07$ & $1.41 \mathrm{E}-05$ & $2.32 \mathrm{E}-05$ \\
\hline
\end{tabular}

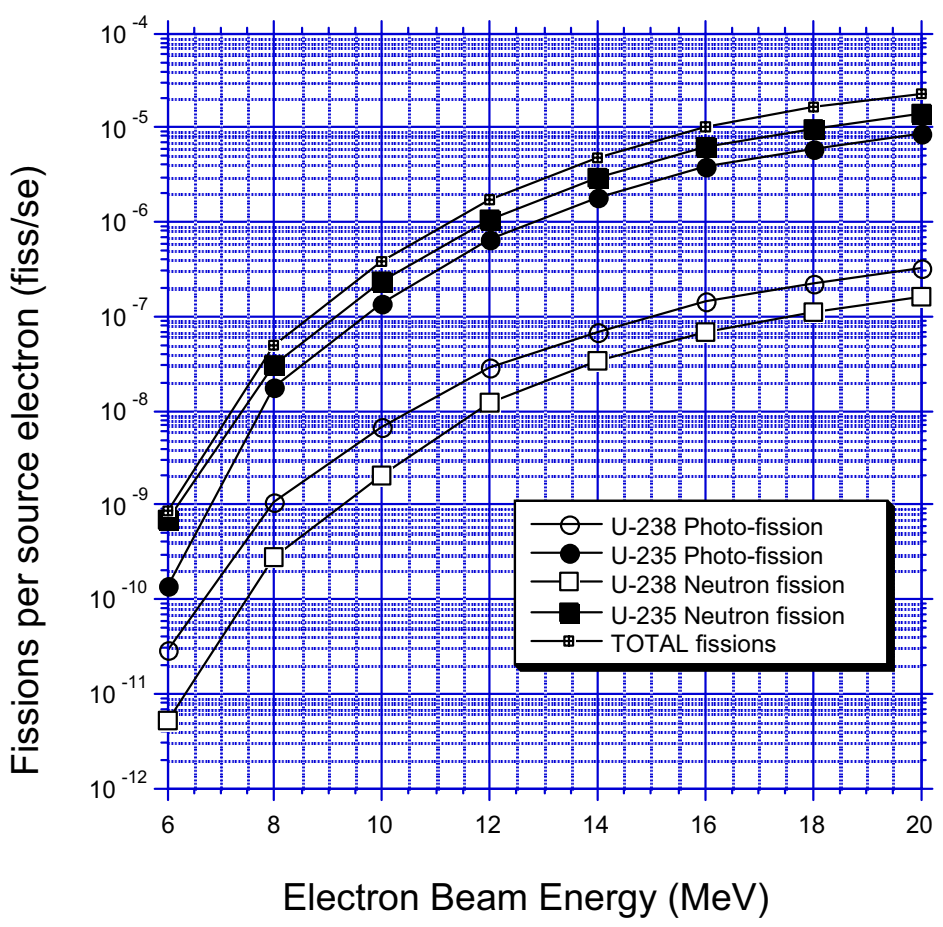

Figure 6-8. Photofission and neutron-induced fission components as a function of electron beam energy for the HEU sphere in the Plywood Calibration Pallet. 
Comparing the fission components between the HEU sphere and HEU plate cases (Table 6-3 and Table 6-4 data), the HEU plates produced approximately twice as many total fissions as the HEU sphere. Relative to the HEU sphere, the HEU plate has a spatially-distributed mass which leads to a higher probability of photonuclear interactions and reduced self-shielding of both photons and neutrons. Despite the fact that the HEU sphere has a slightly larger mass than the plate configuration ( $5 \mathrm{~kg}$ vs $4.8 \mathrm{~kg})$, the HEU plate has an exposed total surface area of approximately $461.5 \mathrm{~cm}^{2}$ versus the sphere surface area of only $200.9 \mathrm{~cm}^{2}$. This larger surface area or distributed mass allows a larger number of bremstrahlung photons to interact with the plate. Similarly, photoneutrons produced in the plywood pallet (from natural deuterium) become thermalized and result in a higher neutron interaction probability in the case of the HEU plate. Hence, the HEU plate geometry is expected to have a higher fission rate component.

\subsubsection{Experimental Evaluation}

To experimentally assess photonuclear nuclear material responses with energetic electron beam energies, experiments have been performed using a $60-\mathrm{Hz}$ pulsed, nominal $6-$ to $25-\mathrm{MeV}$ Linac (Varian L2500) and a 60-Hz pulsed nominal 13- to 20-MeV Linac (Varian CL20). These accelerators were klystron-driven, S-band-type electron Linacs with energy analyzing and beam deflecting bending magnets. A magnetically deflected electron beam was incident on a 2.5 -mm-thick tungsten bremsstrahlung converter to generate the interrogating photons. Both Linac designs allowed for variable energies and pulse widths. The beam current deposited in the converter was monitored. The pulse widths varied from 1 to $1.5 \mu$ s depending of the energy of operation, and the peak beam currents ranged from 7 to $32 \mathrm{~mA}$. Immediately behind the converter is a $5-\mathrm{cm}$ thick piece of aluminum to absorb any transmitted electrons. Four DU plates $(2.7 \mathrm{~cm} \times 15.4 \mathrm{~cm} \times 0.3 \mathrm{~cm})$ were centered on the accelerator centerline two meters in front of the converter. The total mass of all uranium plates is $4.8 \mathrm{~kg}$. The experimental setup using the L2500 is shown in Figure 6-9 where the converter was located within a lead collimator (right side) and several PPNDs are located around the DU at distances ranging from 1 to $2.5 \mathrm{~m}$. The back wall is located about one meter from the depleted uranium. The background responses were determined by repeating each nominal accelerator energy operation without the uranium.

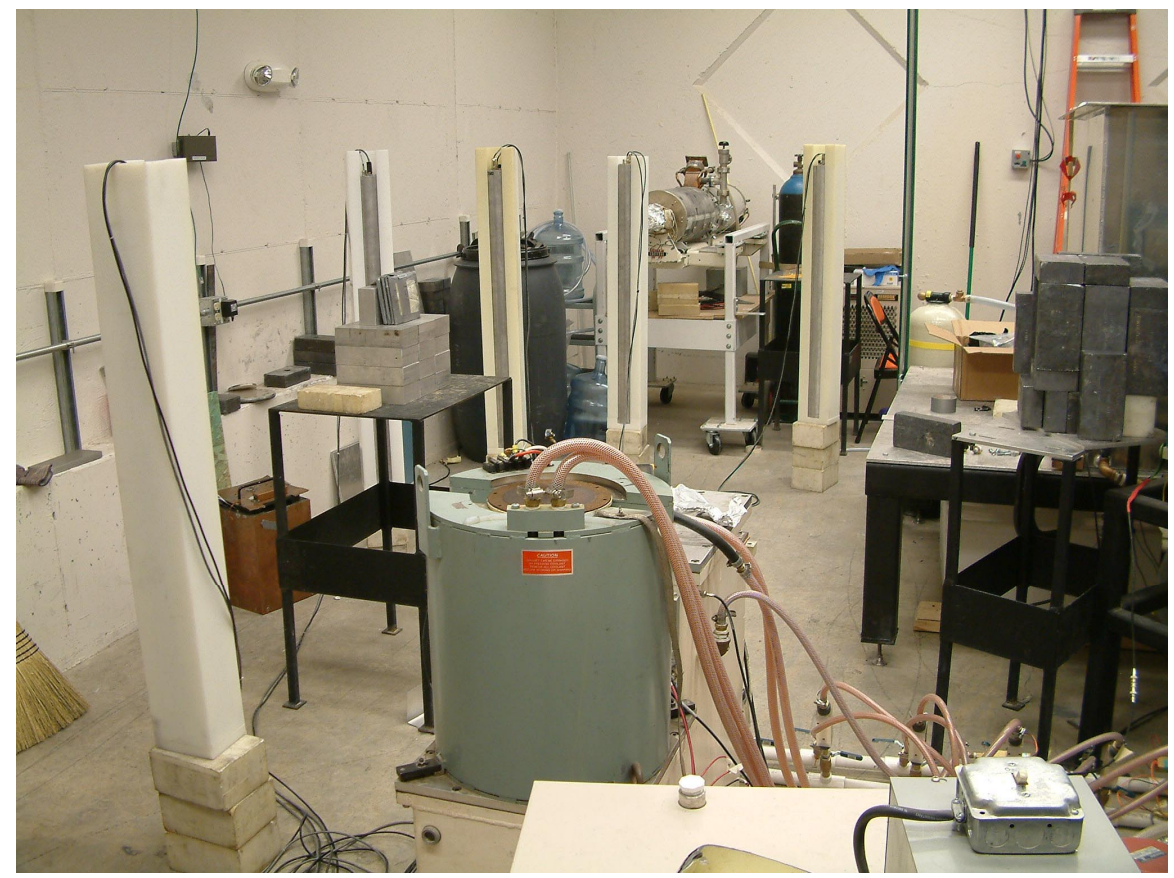

Figure 6-9. Experimental configuration with a L2500 at the IAC. 
Additional experiments were conducted with a CL20 linac in the main Accelerator Hall at IAC (See Figure 6-10). These tests utilized the same experimental test configuration as before; however, this facility provided considerably more floor area (i.e., back wall was about $8 \mathrm{~m}$ from the uranium). This test campaign assessed a different accelerator performance, any facility dependences, and the Plywood Calibration Pallet. Figures 6-10 and 6-11 show the experimental testing configurations.

Figure 6-12 provides delayed neutron count data from both of these experimental campaigns as a function of electron beam energies. Data is provided as delayed neutron counts per second per watt (beam power). As the results indicate, both accelerators provide similar delayed neutron responses for DU that increases with increasing electron beam energy. For cases without DU, it appears that the induced background response is small up to about 18 or $19 \mathrm{MeV}$. For higher energies, the induced background contribution increases with increasing beam energy. This increasing background effect is attributed to some type of room activation requiring focused attention in FY05. The utilization of the Plywood Calibration Pallet showed that no additional background response (i.e., without DU) was induced by the presence of this empty pallet. Also, the nuclear material response with this pallet had similar energy dependence with a reduced magnitude, as expected, primarily due to neutron transport effects. The largest differences (up to three orders of magnitude) between the "with" and "without" (i.e., background) DU responses occur between $14-$ and $20-\mathrm{MeV}$ electron beam energy operations clearly defining a desired optimal range for an inspection. An enhanced resolution of this optimal energy range must involve additional system studies with various shielding materials (i.e., other Calibration Pallets), nuclear materials, and integrated detection technologies. Unfortunately, this favorable higher energy range is not currently allowed by federal regulation for cargo container inspections. However, as discussed in the next section, this may only be an artificial limitation.

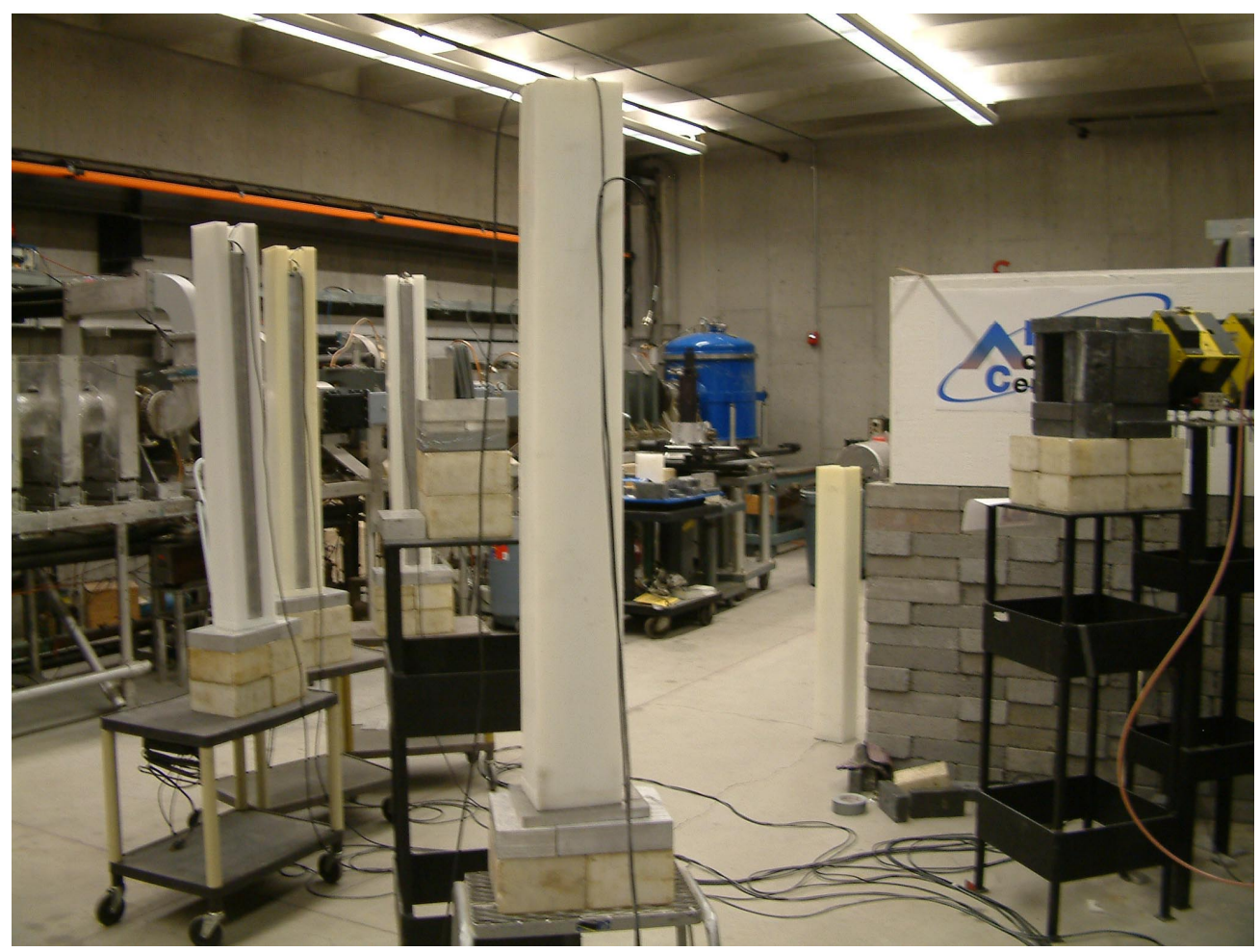

Figure 6-10 Experimental configuration with CL20 in Main Hall at the IAC with DU. 


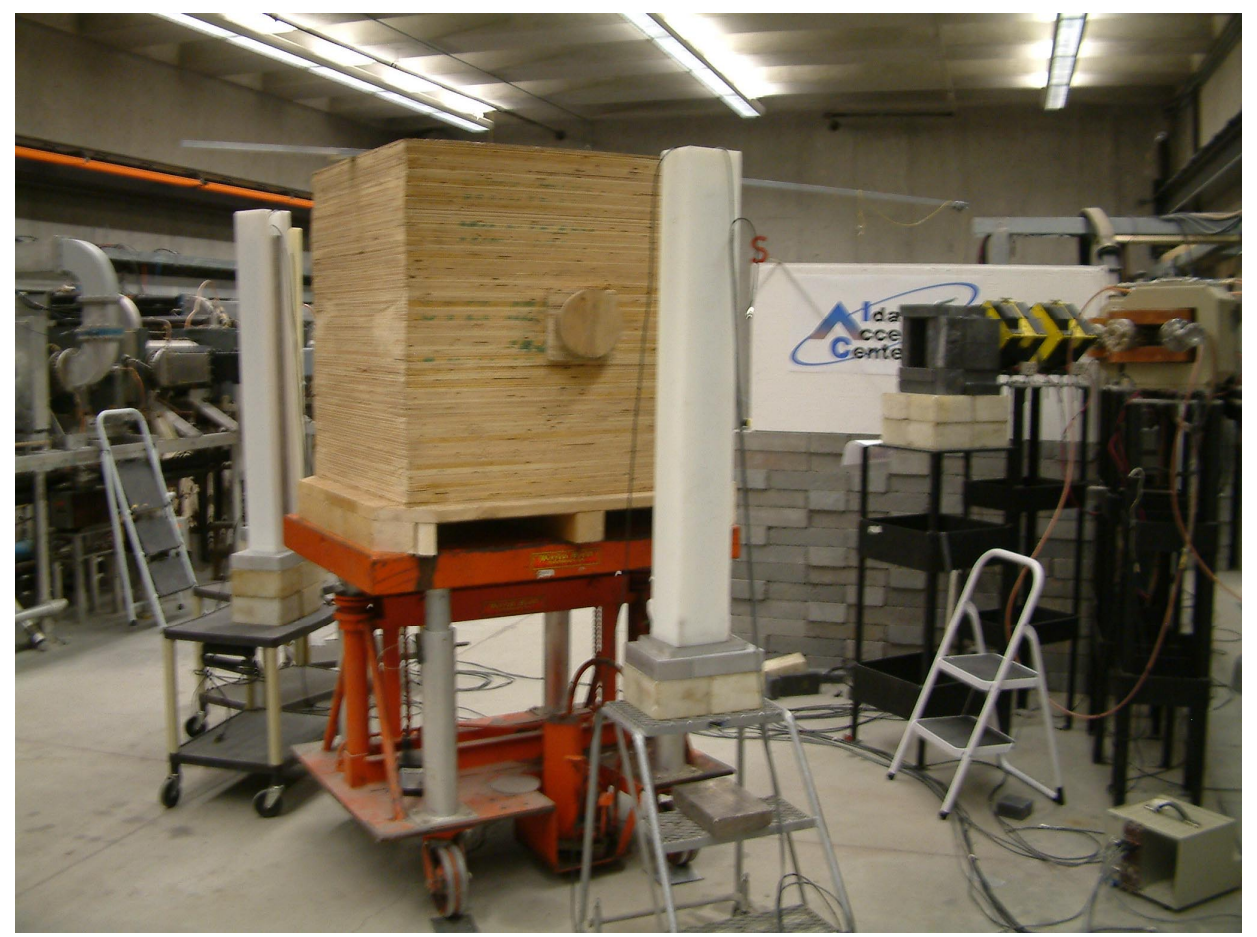

Figure 6-11 Experimental configuration with CL20 in Main Hall at the IAC with the Wood Calibration Pallet.

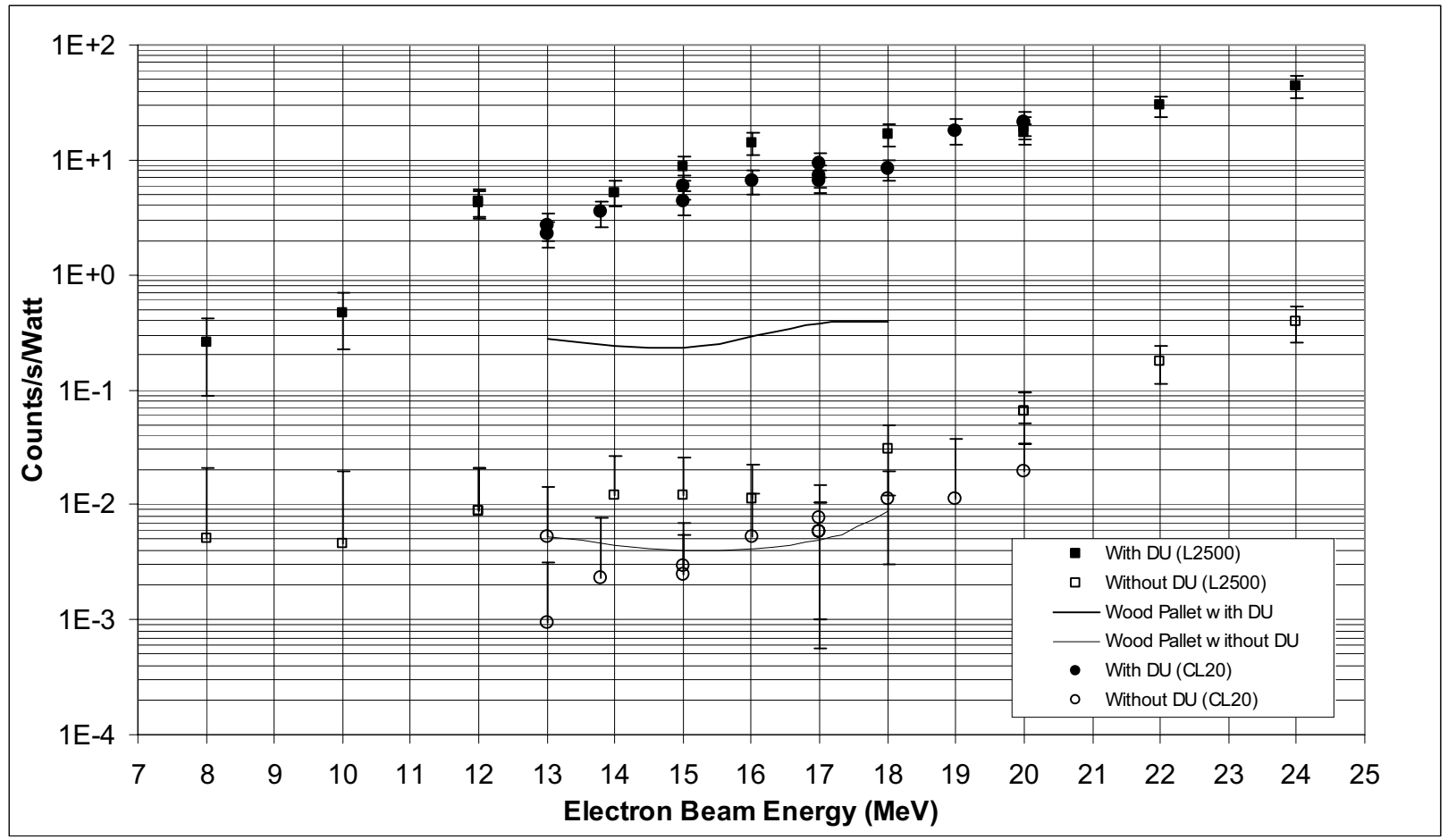

Figure 6-12. Delayed neutron counts (at $2 \mathrm{~m}$ from DU) for various Linacs and shield configurations. 
6.3.2.1 Federal Requirements and Energy Limitation for Food and People. The current federal limitation for machine-generated sources that could be used for food inspection is set forth in 21CFR-179.21. Section A-4 limits the electron beam energy as follows: "Machine sources producing $\mathrm{X}$-ray radiation at energies no greater than 10 million electron volts $(\mathrm{MeV})$." The dose limit is also given in this section as 0.5 gray ( $50 \mathrm{rad})$ per inspection that appears to be a reasonable dose limit for cargo inspection. For comparisons, most of today's imaging systems deliver doses of about $50 \mu$ gray ( 5 millirad) which is about $10^{-4}$ of the 0.5 gray federal limit. If the current imaging systems were to increase their exposure and beam energy, they could readily provide not only imaging but also significant nuclear material detection.

Information in the Federal Register Vol. 66, No. 69, page 18539 indicates that the $10-\mathrm{MeV}$ limit was primarily based on the following three papers:

"Food safety aspects relating to the application of X-ray surveillance equipment: Memorandum from a WHO meeting,” Bulletin of the World Health Organization(WHO), Vol. 31, 1990, pp. 297-301

C.A. Wakeford and R. Blackburn, "Induction and Detection of Radioactivity in Foodstuffs Irradiated with $10 \mathrm{MeV}$ Electrons and X-rays," Radiation Physics and Chemistry, Vol. 38, No. 1, 1991, pp. 29-38

D.J. S. Findley, T. V. Parson and M. R. Sense "Experimental Electron Beam Irradiation of Food and the Induction of Radioactivity," Applied Radiation and Isotopes, Vol. 43, 1992, pp. 567-575

Of these references, all considered and accepted higher energy electron beam operations $(>10 \mathrm{MeV}$ and up to $1 \mathrm{MRad}$ ), and even the WHO reference that established the $10-\mathrm{MeV}, 0.5$-Gray operational limit also concluded:

"However, this conclusion is not intended to preclude other safe surveillance systems designed to operate at a higher energy level or dose. In such cases, assurance should be provided that, at the point of consumption, food would not contain a measurably detectable amount of induced radioactivity."

Also, note that the commonly accepted yearly dose limit for all radiation workers is 5 Rem (or 5 Rad) as set by 10CFR-20. This well established limit, which is one-tenth of an allowable container inspection dose, has shown no biological risk from day-to-day exposures. If a stowaway is hiding in a cargo container, then they are already assuming a risk that could easily exceed the risk of receiving a 5 Rem exposure. The dose and energy limit established in 21CFR-179 may still be limiting for nuclear material detection applications, and with the renewed importance in active interrogation for cargo screening to address homeland security needs, the federal energy limit for machine-generated sources will probably need to be reevaluated.

\subsubsection{Energy Assessment Summary}

Based on experimental and analytical results presented in this report, LINAC operations above 10 $\mathrm{MeV}$ are showing greatly enhanced nuclear material detection capabilities. An increased factor up to $\sim 10^{3}$ per source electron can be seen from increasing the energy of the LINAC operation from $8 \mathrm{MeV}$ to 20 $\mathrm{MeV}$. The determination of the "optimal" electron energy for nuclear material inspection will involve additional system studies that investigate detection responses with differing loading scenarios, different nuclear material types, quantities, and geometries, and potential integration with other WMD detection capabilities and/or detection technologies. 


\subsection{Cargo Loading Effects}

Two different sized pallet configurations with the same nuclear material quantity and position were experimentally assessed for differences in their neutron detection responses. Figure 6-11 shows the multipallet configuration as described in Section 6.1 on the left and the Plywood Calibration Pallet on the right. For each test the electron accelerator (yellow box lower left in multi-pallet configuration photo) beam centerline was aligned with the center with lower Wood Calibration Pallet. The accelerator was operated with a nominal $10-\mathrm{MeV}$ electron beam energy, had a pulsing rate of $125 \mathrm{~Hz}$ (i.e., $8 \mathrm{~ms}$ between accelerator pulses). Four 1.2-kg, rectangular pieces of depleted uranium (DU) were used as representative nuclear material. Multiple PPNDs (shown on the right in the configuration photos with their polyethylene shrouds) were in a vertical plane that is about $53 \mathrm{~cm}$ from the nearest surface of the Calibration Pallets and about one meter from the DU when it was inserted in the center of the Plywood Calibration Pallet. An unshrouded, "He-3 only" PPND-type detector (termed the "bare" detector and shown [vertically positioned] in the lower right of the multi-pallet configuration) was used to monitor overall thermal neutron emissions.
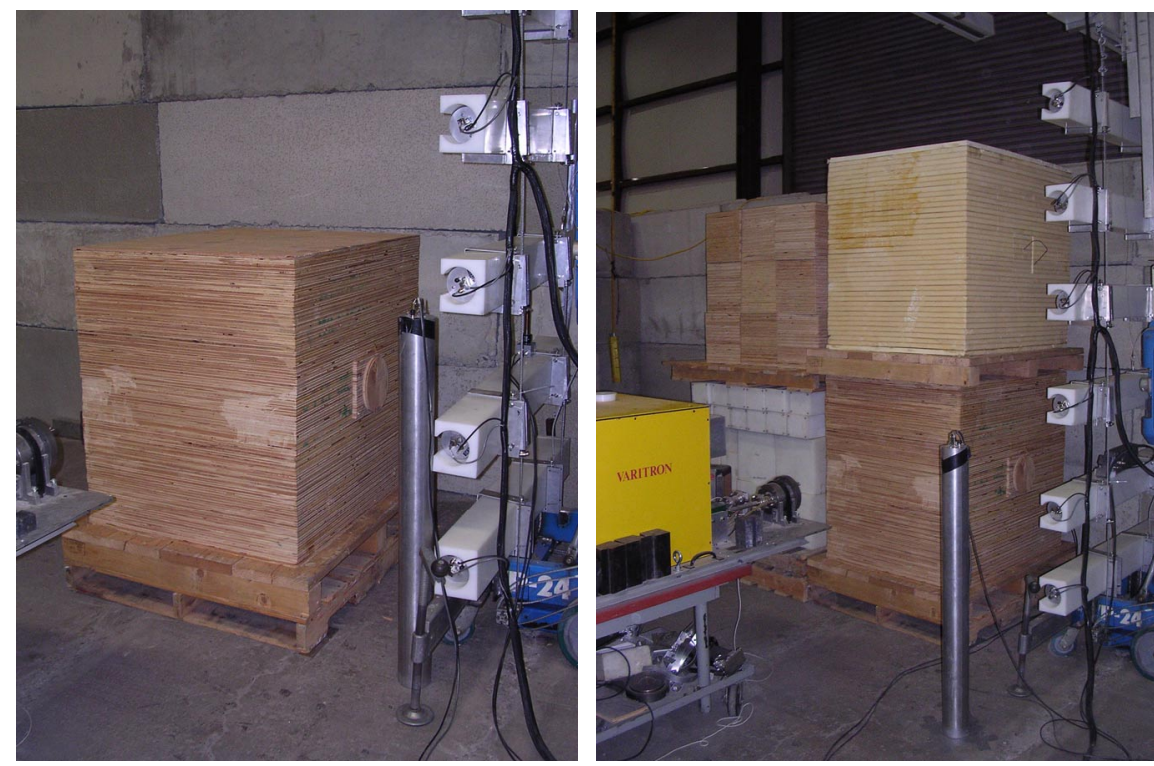

Figure 6-11. Single pallet and multi-pallet (right) test configurations.

The experimental neutron emission results of the single and multiple pallet configurations are shown in Figures 6-12 and 6-13, respectively, for cases involving no DU and with the DU. For comparison the "bare" detector response is also shown for the cases in which concealed DU was used. As can be seen, the specific PPND design enables the rapid recovery after the initial accelerator-induced, prompt photon and neutron "flash" events and then detects only fission representative neutrons, especially after about $1 \mathrm{~ms}$ after each accelerator pulse. Note, the "bare" detector shows a continued contribution of significant "room return" neutrons throughout the pulsing inspection. No noticeable differences can be observed in the PPND's detection response between these two cases; hence, strongly suggesting that the PPND's delayed neutron response is not dependent on the relative cargo container size or composition. In addition, the overall thermal neutron emission response (as shown by the "bare" detector) continues to decrease with time and is orders-of-magnitude larger than the detector's fission neutron detection signal. It is interesting to note that when comparing the bare detector responses for each of these loading configurations, it is observed that the multiple-pallet configuration does yield a slightly higher thermal neutron response emission (see Figure 6-14) but does not appear to have any impact on the DU detection response within the PPND. 


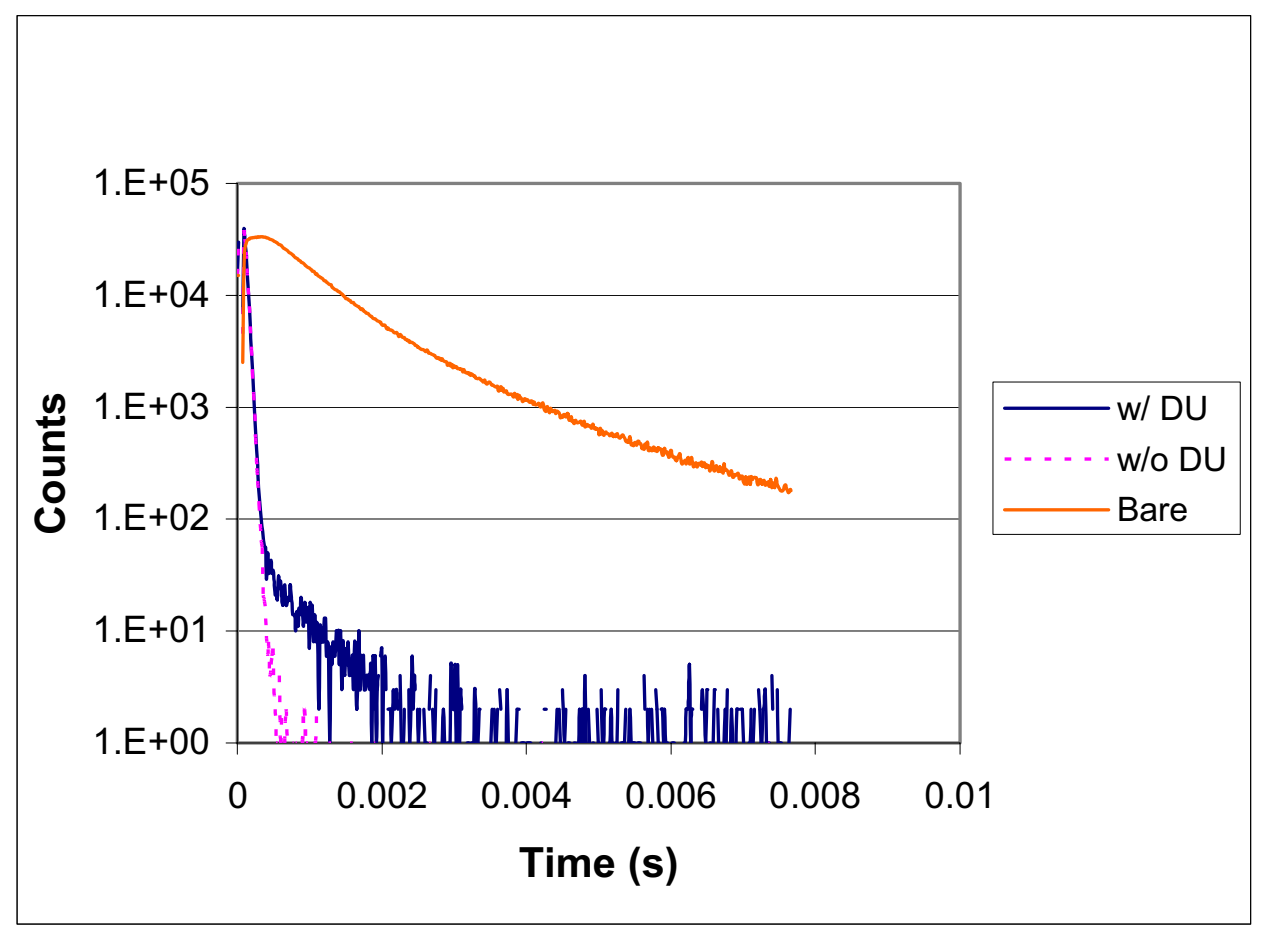

Figure 6-12. Experimental results for the single pallet configuration.

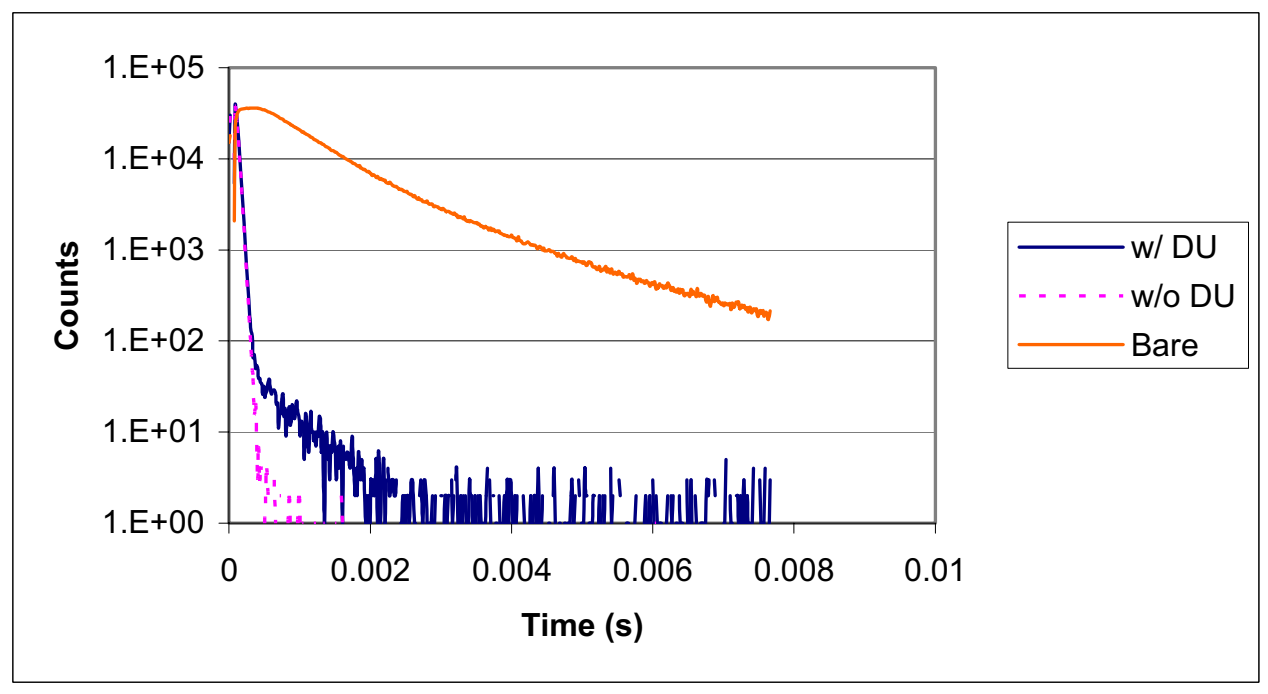

Figure 6-13. Experimental results for the multi-pallet configuration. 


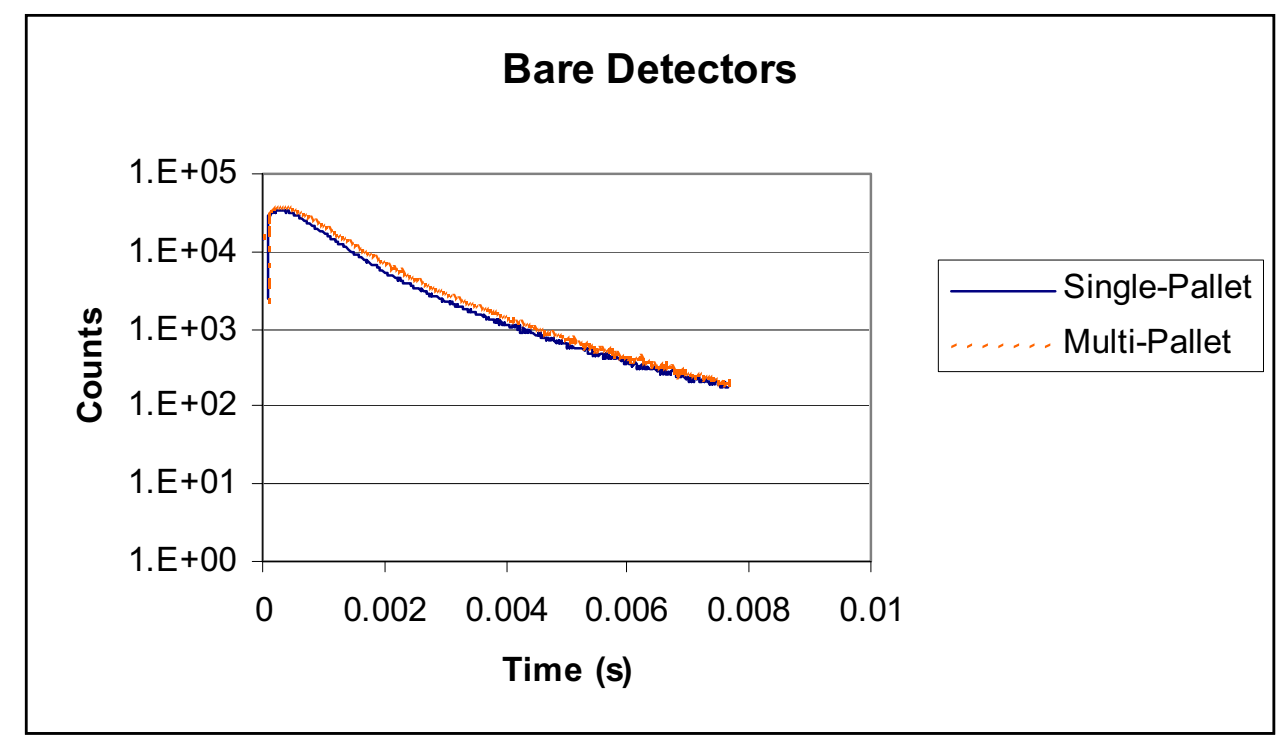

Figure 6-14. Comparison of the "bare" detector responses for the two pallet configurations.

\subsection{Photon Beam Hardening}

For a given high energy photon interrogation, the effect of placing a material directly in front of the electron/photon converter to help "tailor," or attenuate, the resulting source photons (i.e., bremsstrahlung) for reduced doses or specific induced fission needs has been studied. The purpose of this high energy bremsstrahlung study was to determine if one could attenuate the resulting dose from the lower energy photon component while still maintaining the desired induced photofissions from the higher energy component; hence, provide an energy hardened photon beam. A series of MCNPX calculations were performed by varying the beam attenuator material and size. For each study, the selected beam attenuator consists of a single material and is configured in a right cylindrical shape located immediately downstream from the electron/photon converter. The electron/photon converter is a 2-cm-diameter cylindrical disk having a $0.2-\mathrm{cm}$ thickness. While a fixed converter thickness is used for this study, Section 6.6 presents the effects on bremsstrahlung production as a function of converter thickness for a given electron beam energy. The axial centerline of the converter and attenuator corresponds to the beam centerline. The converter is one meter from the surface of a 2.44-m wide cargo container, and a 5-kg HEU sphere is placed on the beam centerline at the center of this cargo container. Finally, the cargo container is filled with Celotex having an artificially high density of $0.4 \mathrm{~g} / \mathrm{cm}^{3}$ (but is representative of an average cargo container loading). Figure 6-15 shows the geometric sketch of calculational model. 


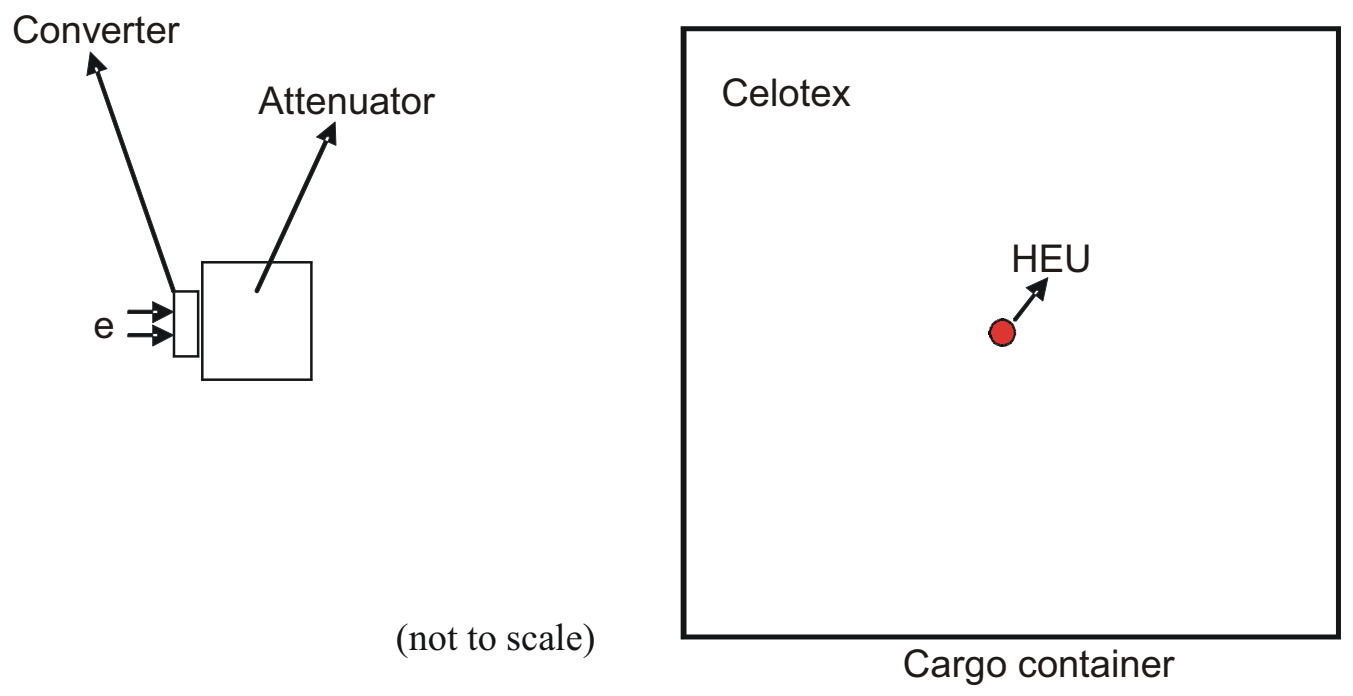

Figure 6-15. Geometric sketch of the calculational model

Electron beam energies of $8,10,15$, and $20 \mathrm{MeV}$ were used in the numerical study and Table 6-5 presents the results. The attenuator material included $\mathrm{D}_{2} \mathrm{O}$, polyethylene (poly), graphite, aluminum (Al), copper $(\mathrm{Cu})$, lead $(\mathrm{Pb})$, and tungsten $(\mathrm{W})$. The size of attenuator ranged from $2.54 \mathrm{x} 2.54-\mathrm{cm}$ to $7.62 \mathrm{x}$ $7.62-\mathrm{cm}$ (i.e., diameter and axial length). The average dose used in this study was calculated over a $10-\mathrm{cm}$ radius at the container surface that is centered on the beam centerline. The induced fissions results correspond to both photofission and neutron induced fissions within the HEU sphere. It is noted that throughout this dose study the neutron dose contribution is minimal. However, the photofissions and induced neutron fission yields are comparable. The "fission/se" column represents the total number of induced fissions per source electron (se). The " $\mathrm{d} / \mathrm{f}$ " column represents the ratio of dose (mrem) to total fissions (i.e., mrem per electron divided by induced fissions per electron). 
Table 6-5. Calculated induced fissions and ratios of dose-to-fission

\begin{tabular}{|c|c|c|c|c|c|c|c|c|c|}
\hline \multirow{2}{*}{\multicolumn{2}{|c|}{ Beam Energy $(\mathrm{MeV})$}} & \multicolumn{2}{|c|}{20} & \multicolumn{2}{|c|}{15} & \multicolumn{2}{|c|}{10} & \multicolumn{2}{|c|}{8} \\
\hline & & fission/se & $\mathrm{d} / \mathrm{f}$ & fission/se & $\mathrm{d} / \mathrm{f}$ & fission/se & $\mathrm{d} / \mathrm{f}$ & fission/se & $\mathrm{d} / \mathrm{f}$ \\
\hline \multicolumn{2}{|c|}{ No attenuator } & 7.7E-6 & $6.3 \mathrm{E}-5$ & $2.1 \mathrm{E}-6$ & $1.1 \mathrm{E}-4$ & $8.8 \mathrm{E}-8$ & $9.8 \mathrm{E}-4$ & $1.2 \mathrm{E}-8$ & $4.2 \mathrm{E}-3$ \\
\hline \multirow{7}{*}{$\begin{array}{l}2.54-\mathrm{x} \\
2.54-\mathrm{cm}\end{array}$} & $\mathrm{D}_{2} \mathrm{O}$ & $7.2 \mathrm{E}-6$ & $6.2 \mathrm{E}-5$ & $2.1 \mathrm{E}-6$ & $1.0 \mathrm{E}-4$ & $8.6 \mathrm{E}-8$ & $9.0 \mathrm{E}-4$ & $1.1 \mathrm{E}-8$ & $4.0 \mathrm{E}-3$ \\
\hline & Poly & $7.2 \mathrm{E}-6$ & $6.2 \mathrm{E}-5$ & $2.0 \mathrm{E}-6$ & $1.1 \mathrm{E}-4$ & $8.7 \mathrm{E}-8$ & $9.0 \mathrm{E}-4$ & $1.1 \mathrm{E}-8$ & $4.1 \mathrm{E}-3$ \\
\hline & graphite & $6.8 \mathrm{E}-6$ & $6.2 \mathrm{E}-5$ & $1.9 \mathrm{E}-6$ & $1.1 \mathrm{E}-4$ & $7.7 \mathrm{E}-8$ & $9.4 \mathrm{E}-4$ & $1.0 \mathrm{E}-8$ & $3.9 \mathrm{E}-3$ \\
\hline & $\mathrm{Al}$ & $6.5 \mathrm{E}-6$ & $6.0 \mathrm{E}-5$ & $1.8 \mathrm{E}-6$ & $1.0 \mathrm{E}-4$ & $7.5 \mathrm{E}-8$ & $9.0 \mathrm{E}-4$ & $9.5 \mathrm{E}-9$ & $3.9 \mathrm{E}-3$ \\
\hline & $\mathrm{Cu}$ & $3.6 \mathrm{E}-6$ & $6.2 \mathrm{E}-5$ & $1.1 \mathrm{E}-6$ & $1.0 \mathrm{E}-4$ & $4.6 \mathrm{E}-8$ & $8.2 \mathrm{E}-4$ & $5.6 \mathrm{E}-9$ & $3.6 \mathrm{E}-3$ \\
\hline & $\mathrm{Pb}$ & $1.7 \mathrm{E}-6$ & $7.4 \mathrm{E}-5$ & $5.0 \mathrm{E}-7$ & $1.3 \mathrm{E}-4$ & $2.4 \mathrm{E}-8$ & $9.2 \mathrm{E}-4$ & $3.1 \mathrm{E}-9$ & $3.9 \mathrm{E}-3$ \\
\hline & $\mathrm{W}$ & $6.3 \mathrm{E}-7$ & $8.7 \mathrm{E}-5$ & $2.0 \mathrm{E}-7$ & $1.4 \mathrm{E}-4$ & $1.1 \mathrm{E}-8$ & 9.9E-4 & $1.4 \mathrm{E}-9$ & $4.2 \mathrm{E}-3$ \\
\hline \multirow{7}{*}{$\begin{array}{l}5.08-\mathrm{x} \\
5.08-\mathrm{cm}\end{array}$} & $\mathrm{D}_{2} \mathrm{O}$ & $6.8 \mathrm{E}-6$ & $6.0 \mathrm{E}-5$ & $2.0 \mathrm{E}-6$ & $1.0 \mathrm{E}-4$ & $8.2 \mathrm{E}-8$ & $8.6 \mathrm{E}-4$ & $1.0 \mathrm{E}-8$ & $3.8 \mathrm{E}-3$ \\
\hline & Poly & $6.9 \mathrm{E}-6$ & $6.0 \mathrm{E}-5$ & $2.0 \mathrm{E}-6$ & $1.0 \mathrm{E}-4$ & $8.0 \mathrm{E}-8$ & 8.9E-4 & $1.0 \mathrm{E}-8$ & $3.9 \mathrm{E}-3$ \\
\hline & graphite & $6.2 \mathrm{E}-6$ & $5.9 \mathrm{E}-5$ & $1.9 \mathrm{E}-6$ & $9.6 \mathrm{E}-5$ & 7.3E-8 & 8.4E-4 & $9.0 \mathrm{E}-9$ & $3.7 \mathrm{E}-3$ \\
\hline & $\mathrm{Al}$ & $5.6 \mathrm{E}-6$ & $5.7 \mathrm{E}-5$ & $1.6 \mathrm{E}-6$ & $9.4 \mathrm{E}-5$ & $6.5 \mathrm{E}-8$ & $8.2 \mathrm{E}-4$ & $8.1 \mathrm{E}-9$ & $3.5 \mathrm{E}-3$ \\
\hline & $\mathrm{Cu}$ & $1.8 \mathrm{E}-6$ & $6.0 \mathrm{E}-5$ & $5.4 \mathrm{E}-7$ & $9.6 \mathrm{E}-5$ & $2.3 \mathrm{E}-8$ & 7.5E-4 & $2.9 \mathrm{E}-9$ & $3.2 \mathrm{E}-3$ \\
\hline & $\mathrm{Pb}$ & $3.7 \mathrm{E}-7$ & $9.2 \mathrm{E}-5$ & $1.2 \mathrm{E}-7$ & $1.5 \mathrm{E}-4$ & $6.3 \mathrm{E}-9$ & $1.0 \mathrm{E}-3$ & $8.6 \mathrm{E}-10$ & $4.2 \mathrm{E}-3$ \\
\hline & W & $6.6 \mathrm{E}-8$ & $1.1 \mathrm{E}-4$ & $1.9 \mathrm{E}-8$ & $2.1 \mathrm{E}-4$ & $1.2 \mathrm{E}-9$ & $1.2 \mathrm{E}-3$ & $1.8 \mathrm{E}-10$ & $4.6 \mathrm{E}-3$ \\
\hline \multirow{7}{*}{$\begin{array}{l}7.62-\mathrm{x} \\
7.62-\mathrm{cm}\end{array}$} & $\mathrm{D}_{2} \mathrm{O}$ & $6.4 \mathrm{E}-6$ & $5.9 \mathrm{E}-5$ & $1.9 \mathrm{E}-6$ & $9.8 \mathrm{E}-5$ & $7.7 \mathrm{E}-8$ & $8.3 \mathrm{E}-4$ & $9.6 \mathrm{E}-9$ & $3.6 \mathrm{E}-3$ \\
\hline & Poly & $6.5 \mathrm{E}-6$ & $5.9 \mathrm{E}-5$ & $1.8 \mathrm{E}-6$ & $1.0 \mathrm{E}-4$ & $7.8 \mathrm{E}-8$ & 8.3E-4 & 9.3E-9 & $3.8 \mathrm{E}-3$ \\
\hline & graphite & $5.8 \mathrm{E}-6$ & $5.6 \mathrm{E}-5$ & $1.7 \mathrm{E}-6$ & $9.2 \mathrm{E}-5$ & $6.7 \mathrm{E}-8$ & $7.8 \mathrm{E}-4$ & $8.2 \mathrm{E}-9$ & $3.4 \mathrm{E}-3$ \\
\hline & $\mathrm{Al}$ & $4.8 \mathrm{E}-6$ & $5.5 \mathrm{E}-5$ & $1.4 \mathrm{E}-6$ & $9.2 \mathrm{E}-5$ & $5.4 \mathrm{E}-8$ & 7.9E-4 & $6.9 \mathrm{E}-9$ & $3.3 \mathrm{E}-3$ \\
\hline & $\mathrm{Cu}$ & $8.8 \mathrm{E}-7$ & $5.9 \mathrm{E}-5$ & $2.6 \mathrm{E}-7$ & $9.9 \mathrm{E}-5$ & $1.1 \mathrm{E}-8$ & 7.4E-4 & $1.4 \mathrm{E}-9$ & $3.0 \mathrm{E}-3$ \\
\hline & $\mathrm{Pb}$ & $8.7 \mathrm{E}-8$ & $1.2 \mathrm{E}-4$ & $2.9 \mathrm{E}-8$ & $1.9 \mathrm{E}-4$ & $1.8 \mathrm{E}-8$ & $1.1 \mathrm{E}-3$ & $2.4 \mathrm{E}-10$ & 4.7E-3 \\
\hline & W & $1.0 \mathrm{E}-8$ & $1.1 \mathrm{E}-4$ & $2.9 \mathrm{E}-9$ & $2.1 \mathrm{E}-4$ & $1.5 \mathrm{E}-8$ & $1.5 \mathrm{E}-3$ & $2.0 \mathrm{E}-11$ & $6.5 \mathrm{E}-3$ \\
\hline
\end{tabular}

Note: all data within $4 \%$ relative error.

Table 6-5 shows that the induced fission yield of an interrogated nuclear material (shielded or unshielded) decreases with increasing attenuator material atomic number and/or greater attenuation lengths. In addition, based on these higher electron beam energies and a given attenuator configuration, an examination of the resulting dose-to-fission ratios show that this ratio is relatively insensitive to the attenuation material used showing that the attenuator effect on dose is similar to that of the induced fissions. The latter implies that while the attenuators do effectively absorb the initially generated, low energy photons that do significantly contribute to an object's overall dose, these attenuators will also contribute to energy downscattering of the higher energy bremsstrahlung component which both reduces the induced fission yields and re-establishes a lower energy dose component. Thus, any attempt to "harden" a high energy photon beam to reduce the overall interrogated object dose will also reduce the induced fission rate within any nuclear material that may be present.

\subsection{Photon Collimator Design Study}

The original goal of this task was to develop a collimator design that would focus the beam radiations in the forward direction and attenuate large-angle scattered gamma and neutron radiations emanating from the electron/photon converter by considering various shield materials and thickness. The parametric design task quickly became a much more complex task with the additional goal of trying to 
maximize the photofission and neutron fission rates in a hypothetical interrogated object, such as the Celotex Calibration Pallet containing a 5-kg HEU sphere. The additional scope required additional parametric studies to include converter and collimator material and geometric variables, as well as variables involving the electron beam characteristics. The following design variables have been considered in the collimator design parametric studies: target material, target thickness, electron beam radius, electron beam energy, collimator material, collimator thickness, and collimator flare angle.

In order to limit the scope of the parametric calculations, several variables were arbitrarily fixed. These included the use of the Celotex Calibration Pallet (with an specified average $0.4 \mathrm{~g} / \mathrm{cc}$ loading density, a 5-kg HEU sphere in the center of the pallet, axial length of the collimator (approximately 30$\mathrm{cm}$ total length), and the location of the target near the axial center of the collimator.

Initial results from two of the parametric studies; one involving the target material and the other involving the electron beam radius, indicated that these variables could be fixed without significant impact to the collimator design. Target materials spanning the Z-range from $\mathrm{D}_{2} \mathrm{O}$ to uranium were assessed as potential converter materials. As expected, the results verified that the higher-Z materials produced the largest fraction of high-energy bremstrallung photons. These converter results also indicated that no significant bremsstrahlung production advantage was obtained with the use of any element between $Z=67$ and $Z=82$; hence, a tungsten converter was used in these parametric studies. It should be noted that tantalum was as effective as tungsten, and that platinum, iridium, and osmium were superior in suppressing the low-energy bremstrallung. Another study involving the electron beam diameter (0.20-2.0 $\mathrm{cm}$ ), showed no notable sensitivity to the emitted bremstrahlung photon spectra and/or intensity.

The following is a brief synopsis of some of the more important/interesting results of these parametric studies:

1. The maximum photofission rate using a $10 \mathrm{MeV}$ electron beam was determined to occur with a Wconverter having a thickness between 0.05 and $0.10 \mathrm{~cm}$ as indicated by the maximum bremsstrahlung spectra (See Figure 6-16). Similar curves showing increasing converter thickness response with increasing electron beam energies can be obtained for other electron beam energies. Note the effect on the lower energy portions of the bremsstrahlung spectra as the converter thickness is varied. The induced fissioning rate was insensitive to collimator flare angle $\left(1-20^{\circ}\right)$ and collimator material $(\mathrm{Pb}, \mathrm{Al}, \mathrm{Cu})$. In addition, the photofission rate will increase with increasing electron beam energy.

2. The side-lobe gamma dose rate could be effectively reduced with a small collimator flare angle. Flare angles of 1-5 degrees suppressed side-lobe gamma radiation significantly over the 5-90 degree scattering angle range. At angles greater than 90-degrees, dose rate curves tend to converge and are independent of flare angle. Increases in converter thickness decrease the side lobe gamma dose rates. For example, at $6 \mathrm{MeV}$, increasing the target thickness from 0.10 to $1.0-\mathrm{cm}$ can result in up to a 2.64 reduction in side lobe dose rates.

3. A 5.08-cm aluminum slug (or attenuator) placed on the beam axis after the electron/photon converter, but in contact with the converter, will reduce the photon dose rate to the face of the cargo container by filtering out the low-energy bremstrallung component, but does so at the expense of the induced photofissions (See Section 6.5).

4. Collimator flare-angle was independent of electron beam energy $(6,10$, or $15 \mathrm{MeV})$ relative to the curve shape of the photon dose rate versus scattering angle. 


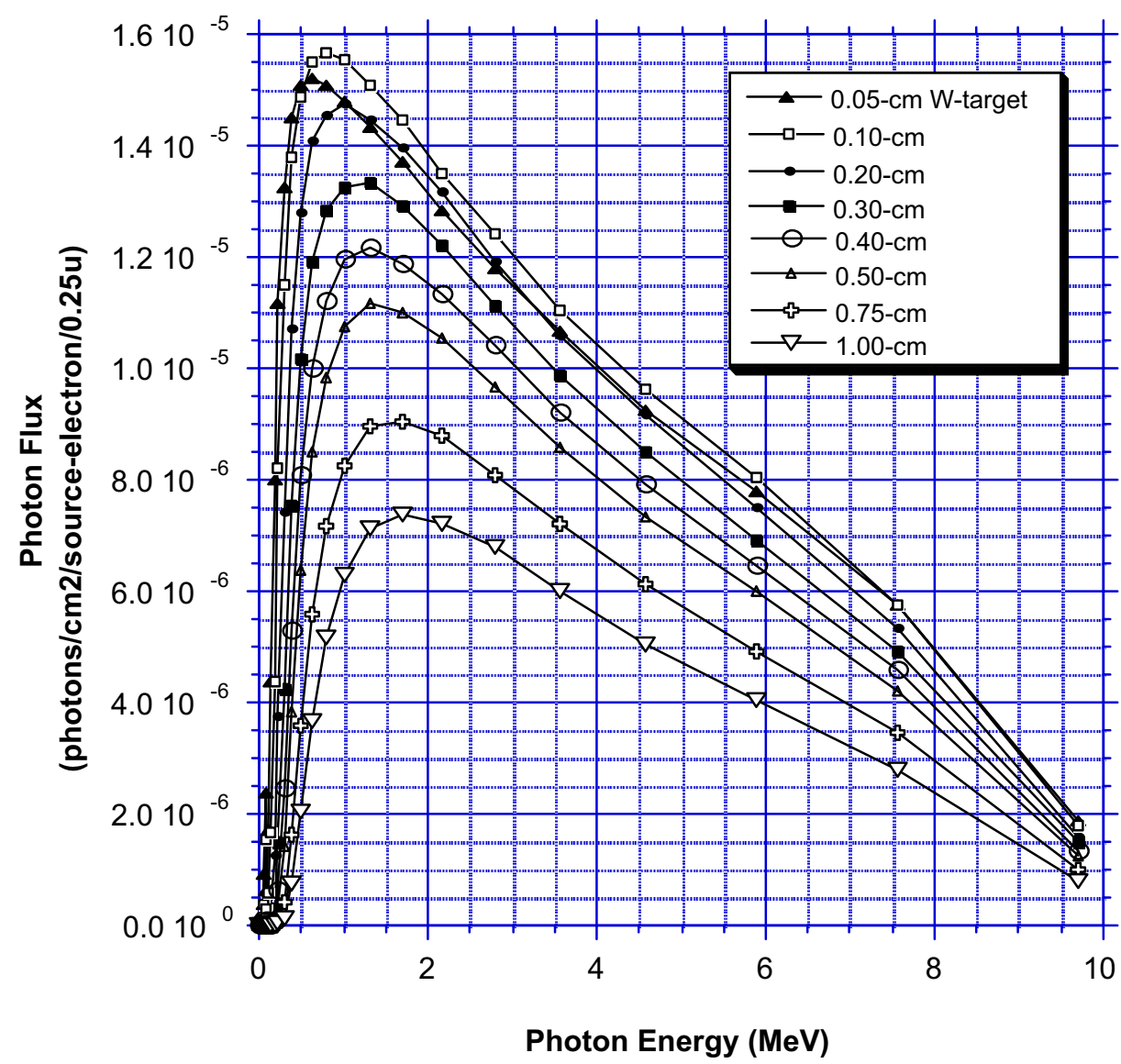

Figure 6-16. Bremsstrahlung spectra for a $10-\mathrm{MeV}$ electron beam incident on various tungsten (W) thicknesses.

5. While photons will dominate the radiation dose to an interrogated object, a factor of two can be seen in the neutron dose rate (at 90-degrees) with increasing collimator flare-angle.

6. Tungsten provided the best collimator photon shield material relative to lead, aluminum, and copper due its large density. Aluminum with its $13-\mathrm{MeV}$ photoneutron threshold is useful in downscattering side-lobe, high-energy photons without producing neutrons over the energy range of 6$13 \mathrm{MeV}$. In addition, aluminum can absorb low-energy photons that would otherwise contribute to side-lobe dose rates and, hence, aluminum is a recommended inner liner for any collimator.

7. Borated-polyethylene and/or lithiated $\left({ }^{6} \mathrm{Li}\right)$ polyethylene provides good neutron shielding.

Based on the above parametric study results three collimator designs have been developed for three different electron beam energies $(6,10$, and $15 \mathrm{MeV})$. An arbitrary external collimator contact dose rate of $100 \mathrm{mrem} / \mathrm{hr} / \mathrm{mA}$ was used to size the specific collimator material thicknesses. Note, the collimator designs affect only the photon and neutron radiation emanating from the $\mathrm{W}$-converter. The resulting skyshine (see Section 6.7) is not shielded by the collimator. As indicated earlier, each of the three collimator designs assumes a total axial length of $30 \mathrm{~cm}$ consisting of a $10-\mathrm{cm}$ section in front of the Wconverter and a $20-\mathrm{cm}$ section thereafter including the collimator conic flare. 
6-MeV Collimator Design: This two-layer design ( $\mathrm{Pb}$-Poly) is simply a cylindrical lead shield tube with an inner and outer diameter of 1.0 and $28 \mathrm{~cm}$, respectively surrounded by a $1-$ to $20-\mathrm{cm}$ thick sleeve of borated-polyethylene (B-poly). The lead shielding is for photon attenuation and the B-poly for neutron shielding. There is no photoneutron production from the lead collimator (only from the $\mathrm{W}$ converter) because the lead isotopes have higher minimum photoneutron threshold energies. Alternatively, in all the collimator designs presented, a higher density material such as tungsten $(19.35 \mathrm{~g} / \mathrm{cc})$ could be substituted for the lead to permit a reduced collimator volume. In addition, the tungsten isotopes have a minimum photoneutron threshold of $6.19 \mathrm{MeV}$ and, like $\mathrm{Pb}$, will not produce neutrons. A collimator flare angle in the lead portion of the collimator could be from 0 to 10 degrees with the smaller flare angles resulting in lower side lobe dose rates.

10-MeV Collimator Design: This three-layer design (Al-Pb-Poly) is similar to the 6-MeV design, except now the photoneutron thresholds of lead and/or tungsten are exceeded and photoneutrons will be produced. It should be noted that side lobe radiation dose rates can be comparable when the electron beam energies are greater than $8 \mathrm{MeV}$. In attempt to mitigate the photoneutron production in the lead or tungsten collimator and to minimize the required outer borated polyethylene shielding, the collimator would have an aluminum inner liner approximately 3.81 to $5.08-\mathrm{cm}$ in thickness. Then, a 30.5 -cm-thick cylindrical lead shield and an outer 5.08 to 10.16 -cm-thick, borated-polyethylene neutron shield would surround the inner aluminum liner. The aluminum liner will not produce photoneutrons because its minimum threshold energy $(13 \mathrm{MeV})$ is above $10 \mathrm{MeV}$. A collimator flare angle of $5^{\circ}$ is chosen to minimize side lobe photon dose rates.

15-MeV Collimator Design: This four-layer design (Al-Cu-Pb-Poly) is similar to the $10-\mathrm{MeV}$ design, except an additional copper $(\mathrm{Cu})$ material layer is now inserted between the inner aluminum sleeve and the lead shield sleeve in order to achieve a graded approach in reducing collimator neutron production. The 13 to $15 \mathrm{MeV}$ bremstrallung photons emitted from the electron/photon converter will induce some neutron production in the aluminum, but the primary purpose of the aluminum liner is to energy downscatter high energy photons. The copper (with a 9.9-MeV photonuetron threshold) is intended to further downscatter and attenuate photons and minimize collimator neutron production. Note, the effects of copper neutron activation may limit its overall effectiveness. Instead of copper, the same effect could be achieved with a thicker aluminum liner, however, the denser copper liner $(8.92 \mathrm{~g} / \mathrm{cc})$ would require less space to deploy. The Al-Cu-Pb-Poly-thickness would be approximately $8.89,8.89,30.5$, and $10.16 \mathrm{~cm}$, respectively. A flare angle of $15^{\circ}$ is chosen to minimize side lobe dose rates.

It is apparent that each collimator shield design is energy dependent and driven by specific design requirements. In all cases the lead material can be replaced by tungsten and result in volume/weight savings. A perfect collimator will attenuate all radiation emanating from the converter in the side lobe directions. Note, one problem not addressed in these collimator designs is the $180^{\circ}$ backscattered electron bremstrahlung radiations. There is no collimator shielding along the beam line axis to attenuate this radiation; however, the end of the accelerator can be shielded (see Figure 4-3). Another type of radiation that can not be eliminated by a collimator design is discussed in the next section.

\subsection{Photon Skyshine Radiation}

As part of the "cabinet-safe" collimator design study, it proved to be of great interest to assess the radiation field around the accelerator system from skyshine. Skyshine, or backscattered photon radiation reflected from the surrounding air and floor back toward the accelerator, would be the minimum amount of radiation dose one would expect for personnel exposure considerations and will be the realistic limit for any final collimator design. 
A dose rate study was performed using a modified MCNP4X model of the accelerator system. The tungsten converter was assumed to be $0.3-\mathrm{cm}$ thick with a surrounding conventional lead collimator. Both bremstrahlung photon and photoneutron production was eliminated in the random walk Monte Carlo simulation if these particles tried to escape the collimator cylindrical sides or the back face. Only the forward-directed bremsstrahlung radiation was allowed to escape via the front flared beam collimator penetration and the front face of the collimator.

The escaping radiations then scattered in the $610-\mathrm{m}$ air volume surrounding the accelerator system and from the floor. Previous studies showed that approximately a $300-\mathrm{m}$ air volume was sufficient to fully backscatter virtually all the photons that were going to backscatter. The dose rates were dominated by the photons and the rate estimates around the accelerator were evaluated as a function of distance (i.e., radius from converter center) and angle from the beam centerline. Scattering angle varied from 0 (forward) to $180^{\circ}$ (backward). The radial distance from the converter was varied from 30 to $400 \mathrm{~cm}$. Dose rates were averaged over conic volumes as a function of distance and angle.

Selected results from this study are presented in Figures 6-17, 6-18, and 6-19 below. Variables in the study included radial distance from the collimator, scattering angle, electron beam energy, and neutron and photon dose rates. Figure 6-17 shows the photon dose rate $(\mathrm{mrem} / \mathrm{hr} / \mathrm{mA})$ for $15 \mathrm{MeV}$ electron beam energy as a function of scattering angle and radial distance. Figure 6-18 is similar, but for the neutron dose rate $(\mathrm{mrem} / \mathrm{hr} / \mathrm{mA})$. Figure 6-19 shows the photon dose rate as a function of scattering angle and electron beam energy at radial distance of $150 \mathrm{~cm}$.

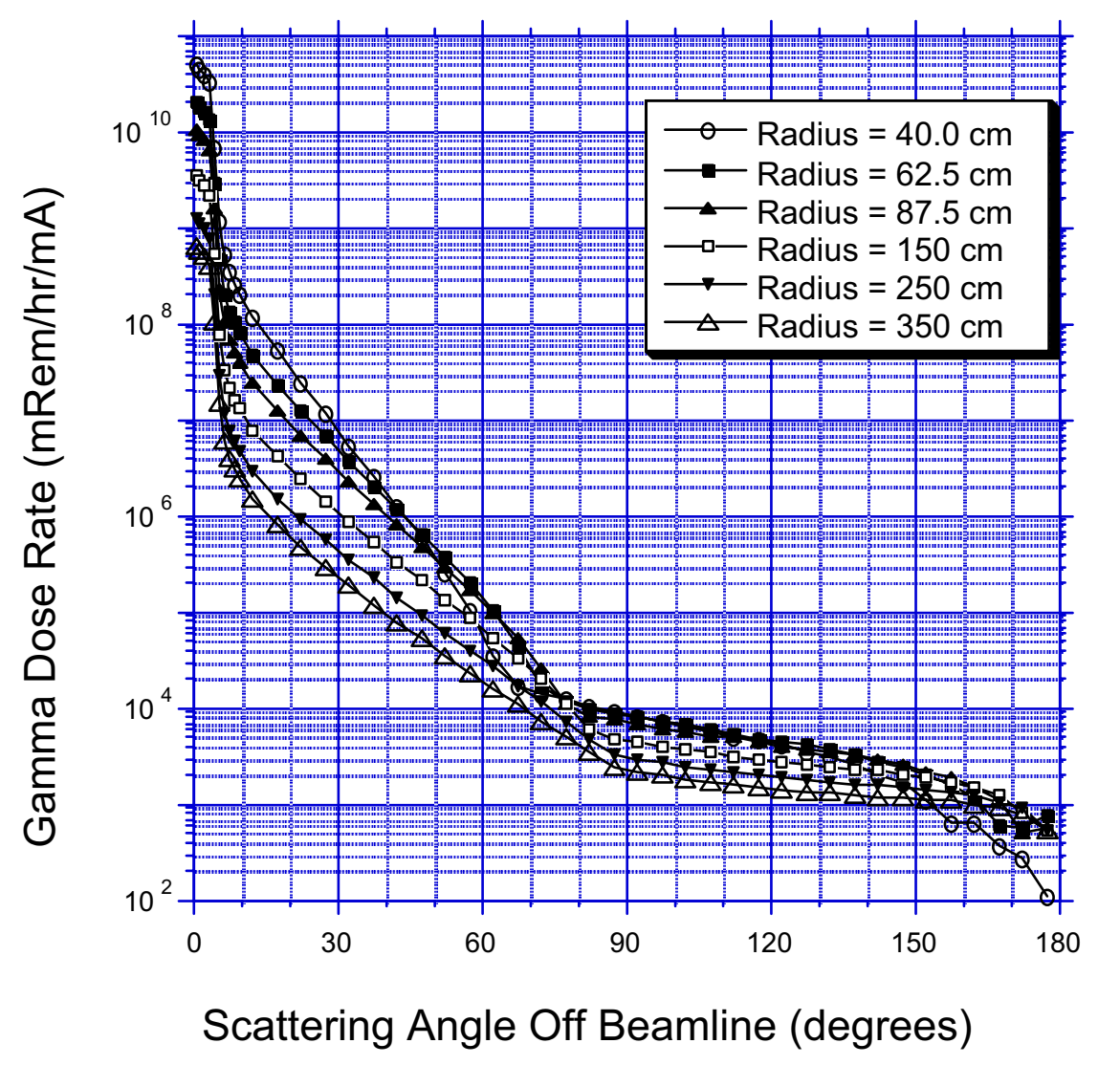

Figure 6-17 Photon dose rate as a function of scattering angle and radial distance from the target (15 MeV electron beam energy). 


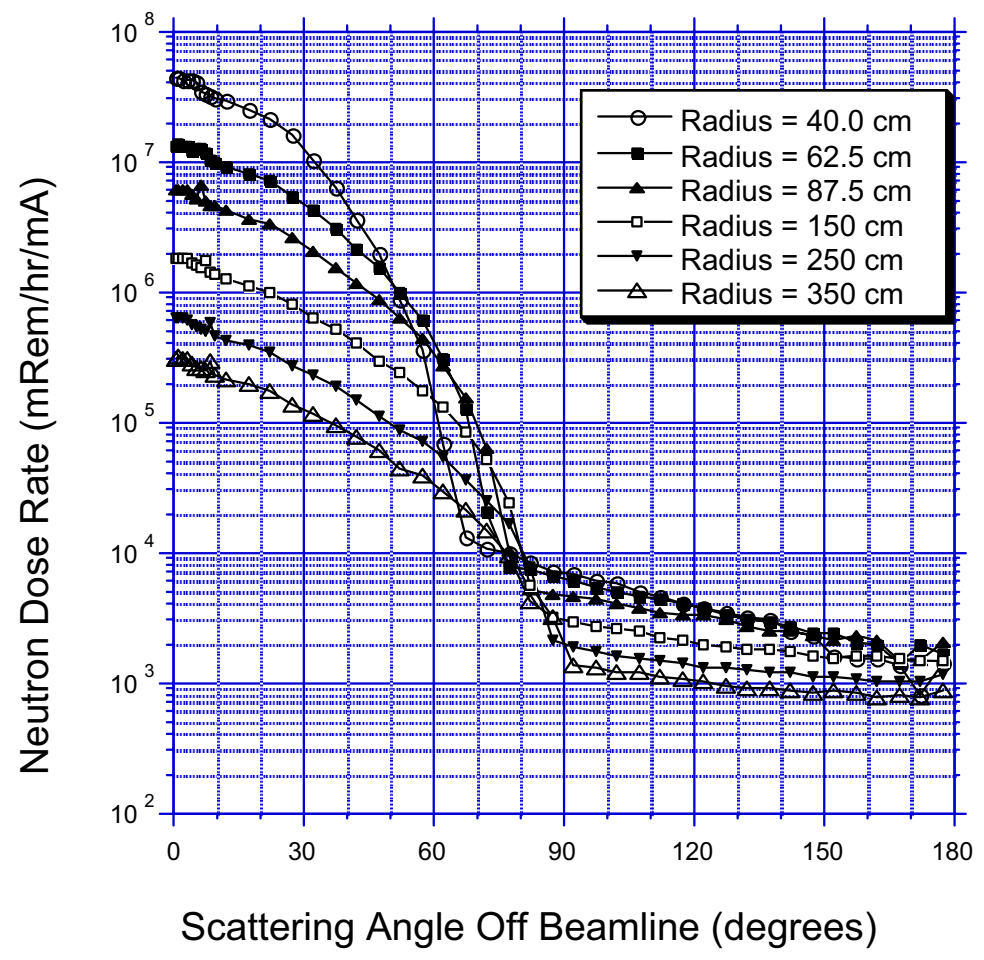

Figure 6-18 Neutron dose rate as a function of scattering angle and radial distance from the target (15 MeV electron beam energy).

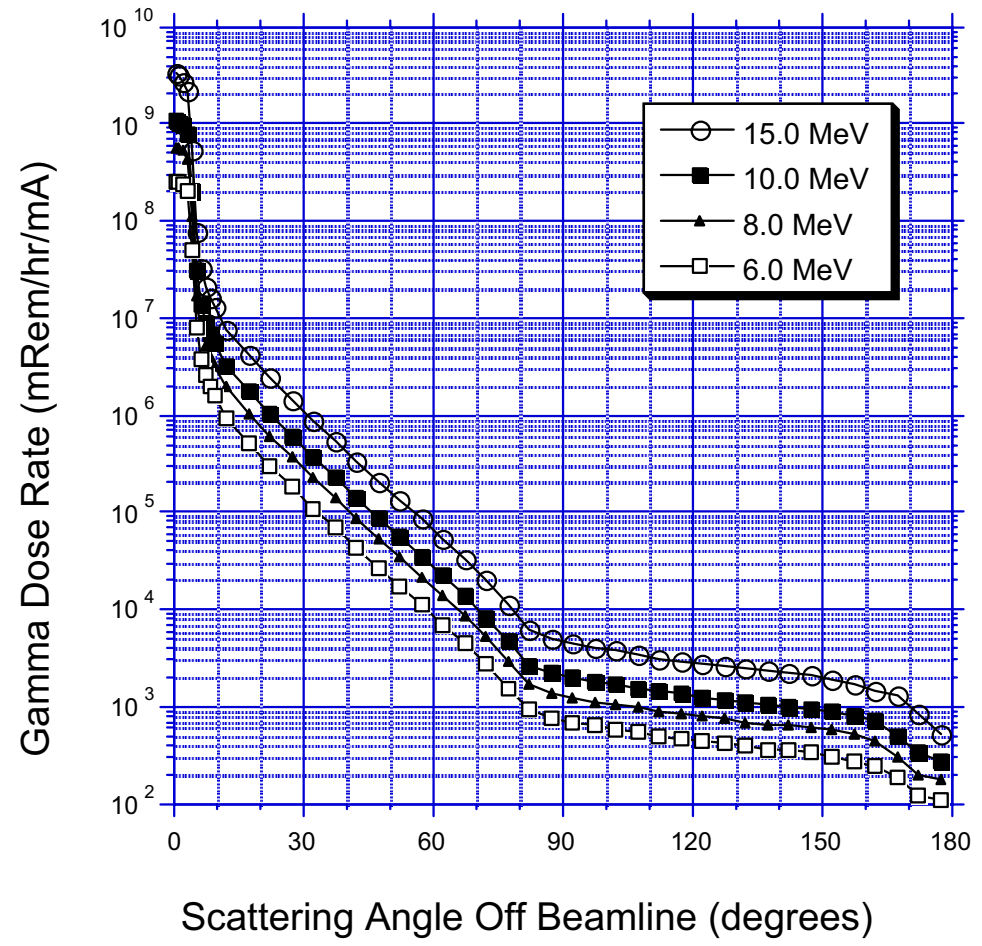

Figure 6-19 Photon dose rate as a function of scattering angle and electron beam energy (150-cm radial distance). 
Figures 6-17, 6-18, and 6-19 show both photon and neutron dose rates fall-off considerably in the backscatter (or greater than $90^{\circ}$ angle) directions. At $15 \mathrm{MeV}$, a skyshine radiation field of less than $\sim 20$ $\mathrm{mrem} / \mathrm{hr}$ is predicted at greater than $90^{\circ}$ with an accelerator operating with a microampere (average) beam current. (This is a beam current similar to a typical $10 \mathrm{-MeV}$ Varitron inspection operation.) As expected, the higher the electron beam energy, the higher the gamma and neutron dose rates per unit of beam current.

\subsection{External Photonuclear Neutron Interrogation}

The following numerical study investigates the potential of converting a portion of the forwarddirected source bremstrahlung photons into interrogating neutrons in order to determine if the increased neutron population into an inspected object would enhance the fission rate in a nuclear material. Using the HEU sphere case (see Section 6.3.1.3) concealed in the Plywood Calibration Pallet, a parametric study was performed as a function of electron beam energy.

Conversion of a portion of the bremstrahlung photons into neutrons was accomplished by placing a 15.24-cm diameter and 15.24-cm long cylinder of heavy water $\left(\mathrm{D}_{2} \mathrm{O}\right)$ directly in front of the electron/photon converter. The $\mathrm{D}_{2} \mathrm{O}$ was assumed to be at room temperature and have a density of 1.1 g/cc. Table 6-6 gives the calculated fission components for the $\mathrm{D}_{2} \mathrm{O}+$ HEU sphere in the Plywood Pallet. These data are plotted in Figure 6-20.

Table 6-6. Fission components for the D2O + HEU sphere in the Plywood Calibration Pallet.

\begin{tabular}{cccccc}
\hline $\begin{array}{c}\text { Electron } \\
\begin{array}{c}\text { Energy } \\
(\mathrm{MeV})\end{array}\end{array}$ & $\begin{array}{c}\text { U-238 } \\
\text { Phofission } \\
\text { (fiss/se) }\end{array}$ & $\begin{array}{c}\text { U-235 } \\
\text { Photofission } \\
\text { (fiss/se) }\end{array}$ & $\begin{array}{c}\text { U-238 } \\
\text { Neutron-induced } \\
\text { fission } \\
\text { (fiss/se) }\end{array}$ & $\begin{array}{c}\text { U-235 } \\
\text { Neutron-induced } \\
\text { fission } \\
\text { (fiss/se) }\end{array}$ & $\begin{array}{c}\text { TOTAL } \\
\text { Fissions } \\
\text { (fiss/se) }\end{array}$ \\
\hline 6 & $1.87 \mathrm{E}-11$ & $9.11 \mathrm{E}-11$ & $8.76 \mathrm{E}-12$ & $1.77 \mathrm{E}-09$ & $1.88 \mathrm{E}-09$ \\
8 & $7.20 \mathrm{E}-10$ & $1.21 \mathrm{E}-08$ & $2.18 \mathrm{E}-10$ & $2.38 \mathrm{E}-08$ & $3.68 \mathrm{E}-08$ \\
10 & $4.61 \mathrm{E}-09$ & $9.49 \mathrm{E}-08$ & $1.73 \mathrm{E}-09$ & $1.77 \mathrm{E}-07$ & $2.78 \mathrm{E}-07$ \\
12 & $1.94 \mathrm{E}-08$ & $4.55 \mathrm{E}-07$ & $8.16 \mathrm{E}-09$ & $7.61 \mathrm{E}-07$ & $1.24 \mathrm{E}-06$ \\
14 & $5.06 \mathrm{E}-08$ & $1.34 \mathrm{E}-06$ & $2.38 \mathrm{E}-08$ & $2.15 \mathrm{E}-06$ & $3.57 \mathrm{E}-06$ \\
16 & $1.04 \mathrm{E}-07$ & $2.79 \mathrm{E}-06$ & $4.93 \mathrm{E}-08$ & $4.40 \mathrm{E}-06$ & $7.35 \mathrm{E}-06$ \\
18 & $1.69 \mathrm{E}-07$ & $4.50 \mathrm{E}-06$ & $8.02 \mathrm{E}-08$ & $7.10 \mathrm{E}-06$ & $1.19 \mathrm{E}-05$ \\
20 & $2.43 \mathrm{E}-07$ & $6.38 \mathrm{E}-06$ & $1.15 \mathrm{E}-07$ & $1.02 \mathrm{E}-05$ & $1.70 \mathrm{E}-05$ \\
\hline
\end{tabular}




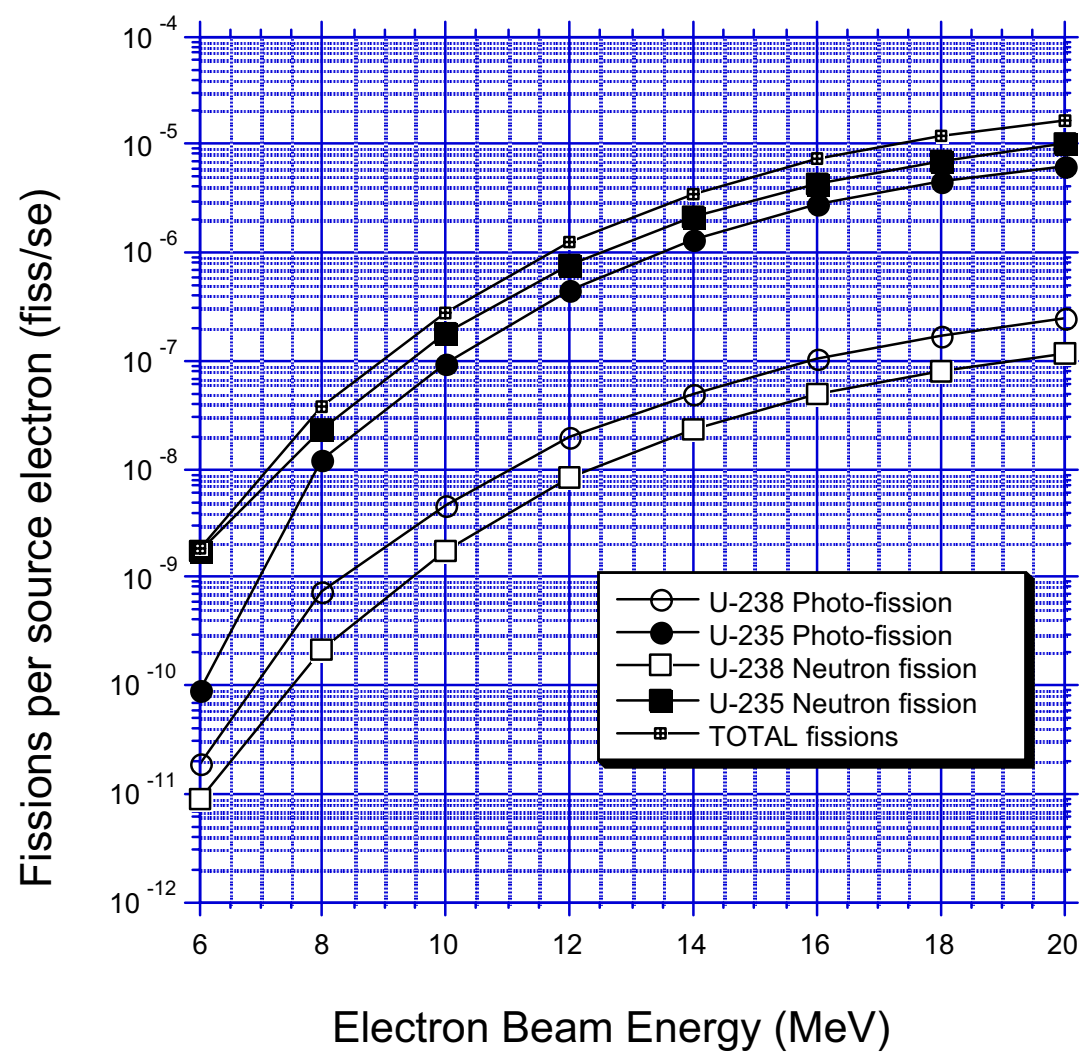

Figure 6-20. Photofission and neutron fission components as a function of electron beam energy for the $\mathrm{D}_{2} \mathrm{O}+\mathrm{HEU}$ sphere in the Plywood Calibration Pallet.

To assess the advantages and/or disadvantages of using an external photoneutron source (i.e., the $\mathrm{D}_{2} \mathrm{O}$ in front of the converter), one must compare the data in Tables 6-4 and 6-6. The data shows that the total fissions decrease by a factor of 1.35 to 1.43 (if we exclude the $6-\mathrm{MeV}$ case) over the $8-20 \mathrm{MeV}$ electron energy range. Hence, for this representative photoneutron production configuration, the use of the $\mathrm{D}_{2} \mathrm{O}$ tends to reduce the intensity of the highly-penetrating bremstrahlung photons at the HEU sphere and that the significantly enhanced neutron intensity from the heavy water does not penetrate the plywood as efficiently as the bremstrahlung photons that do induce the photofission directly. The result for the 10$\mathrm{MeV}$ operation can be seen in Table 6-7. However, if the $\mathbf{D}_{\mathbf{2}} \mathbf{O}$ configuration included a central annular void and selective shielding allowing transmission of the unattenuated, highest energy, forward-directed photons, then an inspection system could favorably utilize both direct photofission and direct neutron-induced interrogation while reducing the forward dose.

Table 6-7. HEU sphere case fission components at $10-\mathrm{MeV}$ operation with and without the $\mathrm{D}_{2} \mathrm{O}$.

\begin{tabular}{cccccc}
\hline $\begin{array}{c}\text { Electron } \\
\text { Energy } \\
(\mathrm{MeV})\end{array}$ & $\begin{array}{c}\text { U-238 } \\
\text { Photofission } \\
\text { (fiss/se) }\end{array}$ & $\begin{array}{c}\text { U-235 } \\
\text { Photofission } \\
\text { (fiss/se) }\end{array}$ & $\begin{array}{c}\text { Neutron-induced } \\
\text { fission } \\
\text { (fiss/se) }\end{array}$ & $\begin{array}{c}\text { U-235 } \\
\text { Neutron- } \\
\text { induced fission } \\
\text { (fiss/se) }\end{array}$ & $\begin{array}{c}\text { TOTAL } \\
\text { Fissions } \\
\text { (fiss/se) }\end{array}$ \\
\hline $\begin{array}{c}10 \\
\left(\text { no } \mathrm{D}_{2} \mathrm{O}\right)\end{array}$ & $4.61 \mathrm{E}-09$ & $9.49 \mathrm{E}-08$ & $1.73 \mathrm{E}-09$ & $1.77 \mathrm{E}-07$ & $2.78 \mathrm{E}-07$ \\
& $6.48 \mathrm{E}-09$ & $1.33 \mathrm{E}-07$ & $2.14 \mathrm{E}-09$ & $2.16 \mathrm{E}-07$ & $3.58 \mathrm{E}-07$ \\
\hline
\end{tabular}


To further help characterize the contribution of the plywood for this case, the neutron flux was calculated directly in front of the Plywood Calibration Pallet on the beam centerline axis and as a function of electron beam energy. Table 6-8 gives the calculated neutron fluxes (neutrons $/ \mathrm{cm}^{2} /$ source electron) at a distance of approximately $75 \mathrm{~cm}$ away from the electron/photon converter (near the pallet surface) as a function of electron beam energy.

Table 6-8. Neutron flux on beam centerline in front of the Plywood Calibration Pallet.

\begin{tabular}{cc}
$\begin{array}{c}\text { Electron Energy } \\
(\mathrm{MeV})\end{array}$ & $\begin{array}{c}\text { Neutron Flux } \\
\left(\mathrm{n} / \mathrm{cm}^{2} / \mathrm{se}\right)\end{array}$ \\
\hline 6 & $1.06 \mathrm{E}-09$ \\
8 & $2.64 \mathrm{E}-09$ \\
10 & $4.82 \mathrm{E}-09$ \\
$10\left(\right.$ no $\left.\mathrm{D}_{2} \mathrm{O}\right)$ & $2.96 \mathrm{E}-10$ \\
12 & $8.15 \mathrm{E}-09$ \\
14 & $1.21 \mathrm{E}-08$ \\
16 & $1.82 \mathrm{E}-08$ \\
18 & $2.58 \mathrm{E}-08$ \\
20 & $3.49 \mathrm{E}-08$ \\
\hline
\end{tabular}

Comparing the $10-\mathrm{MeV}$ case with and without the $\mathrm{D}_{2} \mathrm{O}$, it is clear that the $\mathrm{D}_{2} \mathrm{O}$ does efficiently increase the neutron flux in front of the Plywood Pallet. In fact, it increases the neutron flux going into the pallet by a factor of 16.3. Increasing the electron beam energy and customizing the external photoneutron source should further optimize the concept. 


\section{COMMERCIAL PARTNERSHIPS}

The PPA technology development has been and continues to be pursued with several commercial companies. Two of these commercial partnerships are highlighted in this section. The first involves integration with an existing mobile, $6-\mathrm{MeV}$ radiographic inspection system and the second involves a concept to inspection $100 \%$ of incoming/outgoing maritime cargo containers.

\subsection{ARACOR Eagle}

Advanced Research Applications Corporation (ARACOR) (Sunnyvale, California) specializes in the development of commercial high-energy radiographic inspection devices. While most high energy inspection devices are designed as fixed site applications, ARACOR has developed a mobile system, referred to as the Eagle (shown in Figure 7-1), that can "drive over" a stationary cargo container and image almost any cargo type using a 6-MeV electron accelerator. Such a prototype system has been successfully operated at the Port of Miami for the United States Customs Service. Customs and other international customers have ordered additional systems. ARACOR has worked closely with INL to assess the capability of including a photonuclear detection capability for nuclear material within the Eagle design. Figure 7-2 shows the original concept showing proposed placement of neutron detectors (i.e., PPNDs) on the Eagle. Initial field studies ${ }^{11}$ determined that the $6-\mathrm{MeV}$ operation was marginal for nuclear material detection, and shielded configurations would require higher electron beam energies. ARACOR is pursuing higher electron energy operations versions of the Eagle. Finally, some PPND detectors were field tested in California ${ }^{12}$ with the most recent 6-MeV Eagle design and representative cargo loading.

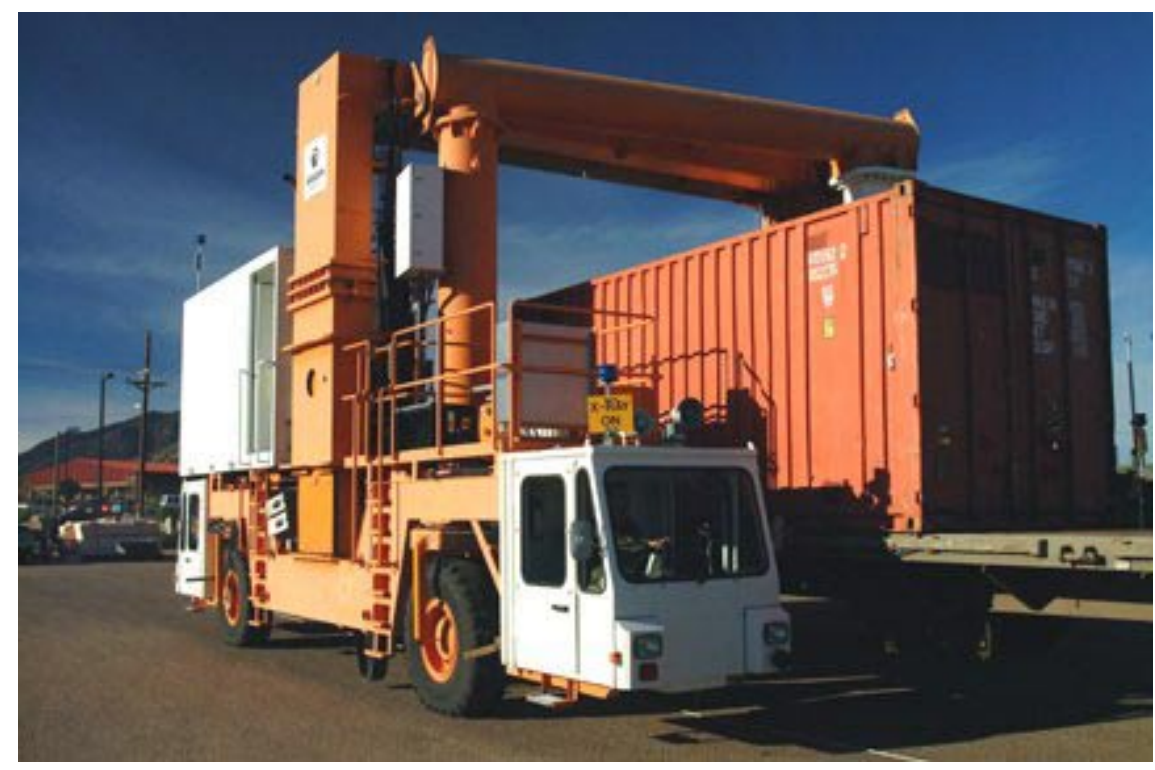

Figure 7-1. ARACOR Eagle Inspection System. 


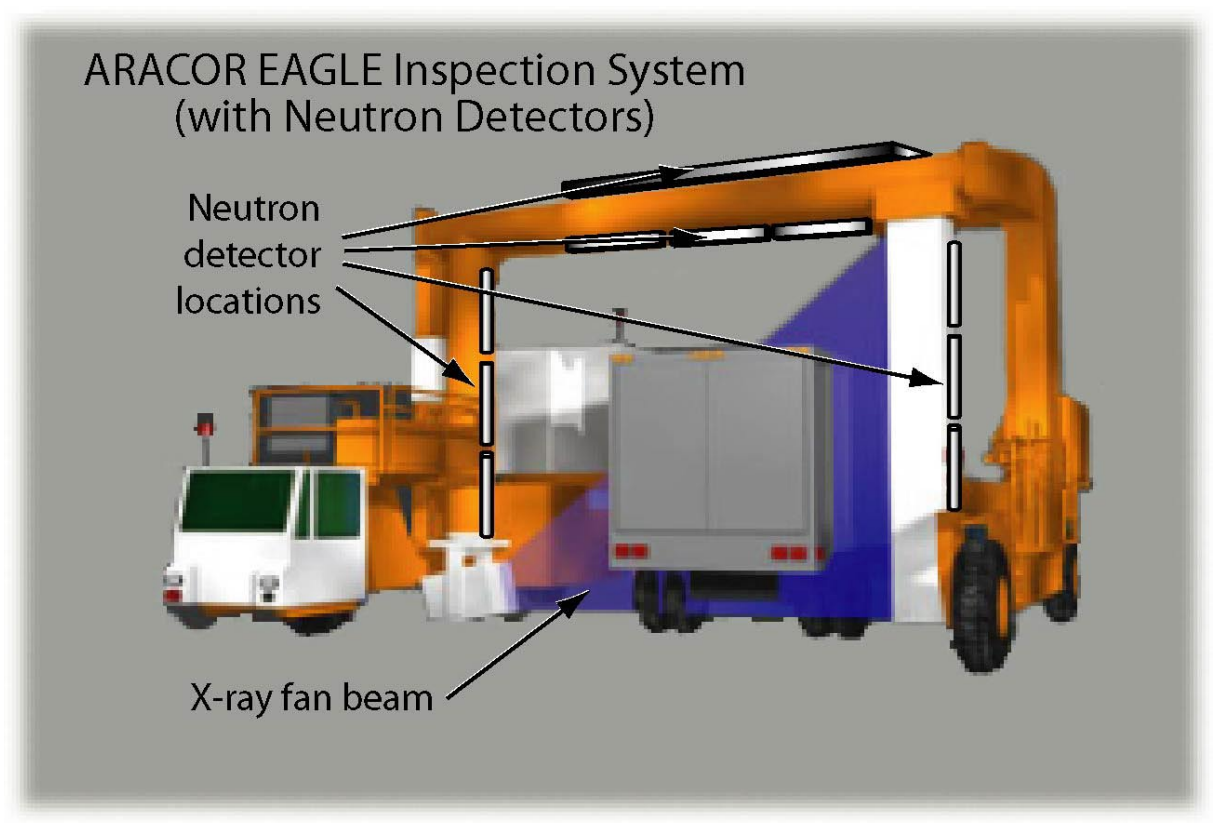

Figure 7-2. Proposed Eagle design for nuclear material detection.

\subsection{PACECO Interceptor}

Pacific Coast Engineering Company (PACECO) (Seattle, Washington) is a major ship-to-shore gantry crane manufacturer. They have over 2500 container handling cranes in 176 seaports in 57 countries. They have been very interested in the development of active inspection systems. Their latest focus is in the development of a container inspection system (referred to as the Interceptor) capable of inspecting 100 percent of all loaded/unloaded cargo containers and rail vehicles. This basic Interceptor concept, using an electron accelerator for imaging and nuclear material detection, is an inspection system in which the inspection process is combined/integrated with the normal cargo container motion between the crane unloading onto a multi-wheeled "buffer" station and the final container positioning onto the staging truck. A seaport deployment of the Interceptor concept is shown in Figure 7-3. PACECO has been working closely with INL and the IAC to support development of an experimental feasibility assessment of the Interceptor concept. A license agreement has already been established with the INL for the use of the PPND design and the IAC has been contracted to build ten PPNDs, fabricate and test an operational testing platform at the IAC, and design and develop an applicable $10-\mathrm{MeV}$ electron accelerator photon source. The completed testing platform, which provides for cargo container motion, is shown in Figure 7-4. 


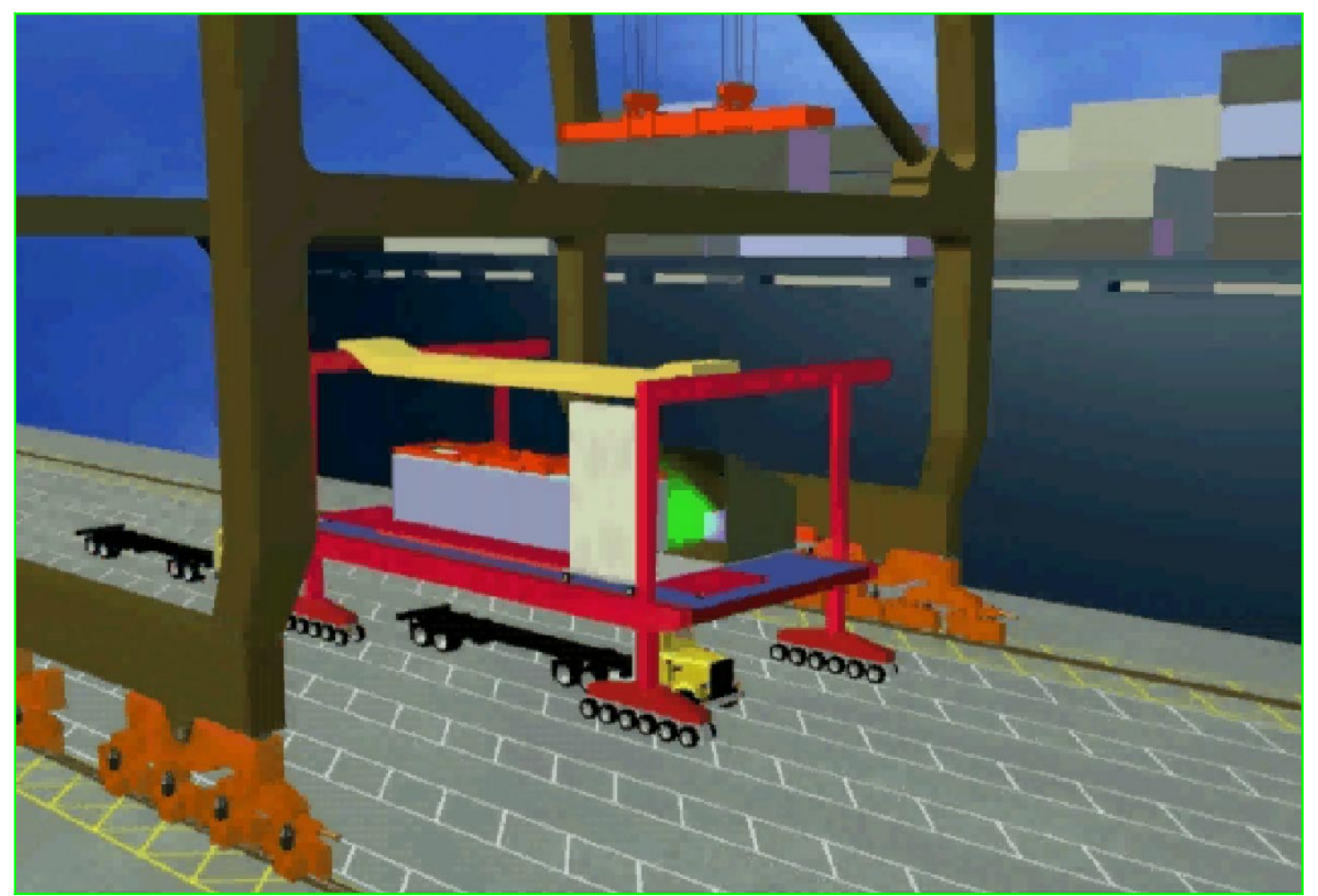

Figure 7-3. Schematic of the PACECO Interceptor inspection concept at a sea port.

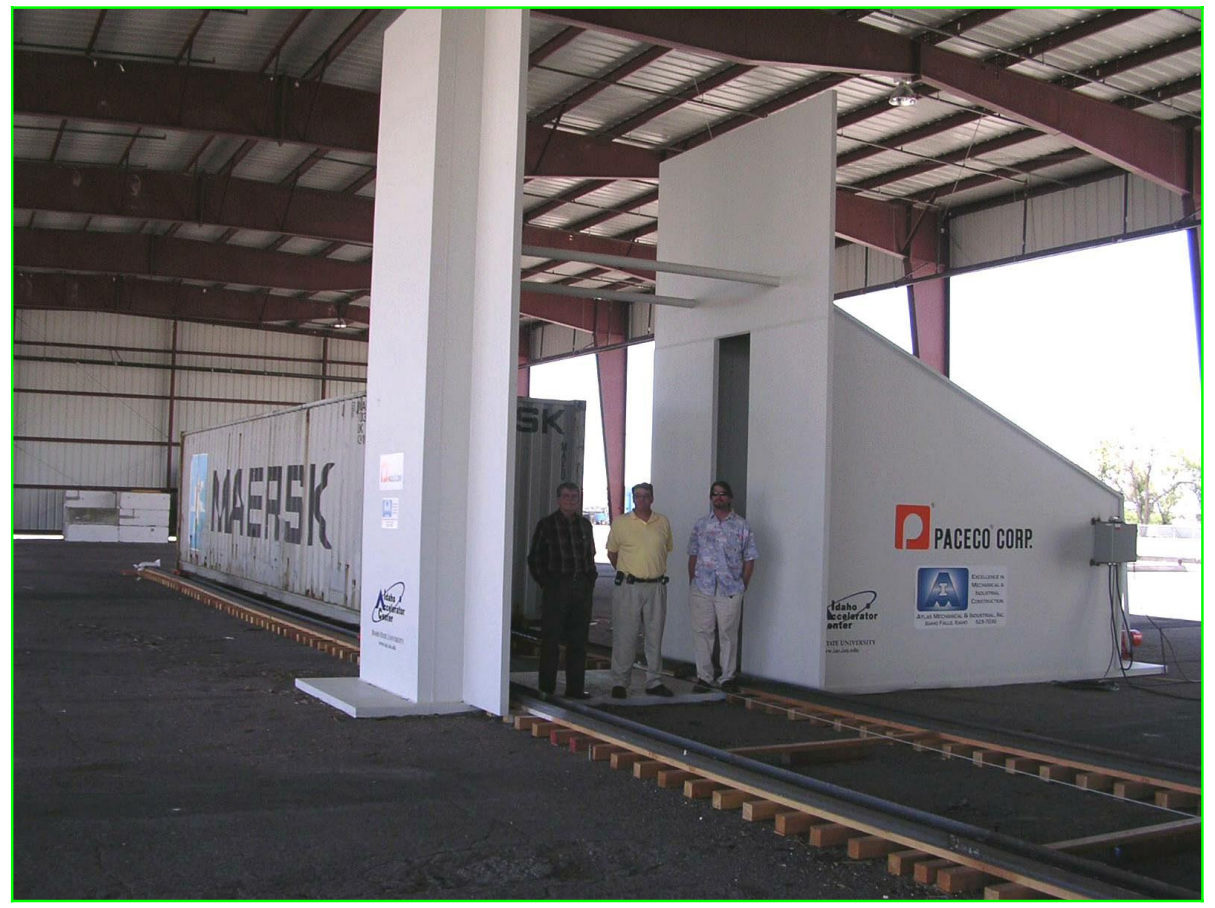

Figure 7-4. The PACECO/IAC cargo container testing platform. 


\section{SUMMARY}

A nuclear material detection system is being developed by INL, LANL, and the ISU/IAC, using PPA technology for the inspection of large transportation vehicles such as trucks, cargo containers and railcar containers. This technology development continues to demonstrate its superior capability in addressing the formidable problem of detecting the presence of shielded nuclear materials, especially $\mathrm{HEU}$, and its flexibility in integrating different detection technologies. The present system uses energetic 8 to $12 \mathrm{MeV}$ photons (but 14-20 MeV appears to enclose an optimal energy range) to provide good cargo material penetration and subsequent photonuclear interactions to stimulate fissions in concealed nuclear materials. The PPA concept is developing into an integrated detection technology system that performs both neutron and gamma-ray (currently being incorporated) detection between accelerator pulses to enable nuclear material detection. In addition, to enable the possibility of rapid, pre-scan inspection applications for most cargo containers, an inexpensive gray scale-type, photon "imaging" or "mapping" system is also being developed and incorporated.

During CY04, major components of a prototype PPA inspection system were built and evaluated. A new electron accelerator (the Varitron II) was designed and assembled at the IAC to address many of the deployment needs related to radiation safety issues and transportability. Operational testing and accelerator development will continue into CY05. Two mobile PPND detector-mounting platforms have been assembled along with an INL-designed, Labview-based acquisition system that provides userfriendly, automated nuclear material detection. In addition, a gamma-ray detection system, using inexpensive Geiger-Muller tubes that work very effectively within an intense pulsed photon environment, is being incorporated directly with the neutron detection system and, for CY05, will be integrated with the Labview-based acquisition system.

To help characterize the performance of the developing PPA inspection system, a set of welldefined pallets of various shield materials have been fabricated. These pallets, referred to as Calibration Pallets, are very effective in establishing benchmark-type numerical and experimental validation and technology comparisons. Measurements with some of these pallets have enabled the development of initial ROC curves for beginning the detection characterization of the PPA inspection system. The CY05 efforts will expand the ROC curves assessments with other calibration pallets, nuclear material quantities and shapes, integrated detection technologies, and electron beam energies.

Numerical and experimental electron beam energy assessments have shown that increased electron beam energies (up to about 24-MeV) will increase the detection capability by orders-of-magnitude. By considering a 4.8-kg depleted uranium signal and induced background responses versus electron beam energy, initial experiments (including the use of the Plywood Calibration Pallet) have indicated that electron beam energies in the range of $14-20 \mathrm{MeV}$ should produce optimal performance with a signal-tonoise ratio performance of up to several 1000. Additional studies must be made in this energy range to further define this optimal energy via a system performance study using the complete set of Calibration Pallets and associated ROC curves to optimize the inspection/detection system and to provide critical data needed for federal regulation justifications.

Numerical predictions using the latest MCNPX code have shown very good numerical comparisons with experimental data involving shielded and unshielded depleted uranium samples. This modeling capability has: (1) enabled response predictions for shielded highly enriched uranium, (2) provided an assessment showing the limited benefits involved in photon beam hardening (i.e., reducing lower energy bremsstrahlung component) when using higher energies photons, (3) allowed development of three energy-dependent photon collimator designs, (4) identified the minimum dose response produced by skyshine radiation (as a function of electron beam energy, off-axis angle and distance from source) from the forward-directed bremsstrahlung source required for cargo inspections, and (5) determined that external (to the cargo), photoneutron-based, neutron sources (that are simply designed) can actually decrease the nuclear material detection capability (i.e., photofission interactions). As a result of this 
CY04 effort, a final photoneutron source design (external to the cargo) has been identified that should be numerically and experimentally assessed to clearly define the advantages/disadvantage of complementary photoneutron sources (external to cargo) for the PPA inspection technology.

Finally, commercialization efforts for the PPA technology development are continuing. Efforts have included an assessment of the ARACOR Eagle design for nuclear material detection that identified detection limitations with its nominal 6-MeV operational design. PACECO has obtained an INL license for the PPND detectors and, in collaboration with the IAC, has designed and developed a cargo container testing facility that allows inspections of loaded cargo container with speeds up to $0.3 \mathrm{~m} / \mathrm{s}$. Finally, several additional companies have express interest in various aspects of the PPA technology component development and applications. 


\section{REFERENCES}

1. J.L. Jones, et al., "Material Identification Technology (MIT): Concept Technical Feasibility Study," INEEL Formal Report, WINCO-1147, September 1993.

2. J.L. Jones, et al.,"Pulsed Photoneutron Interrogation: The GNT Demonstration System," INL Formal Report, WINCO-1225, October 1994.

3. J.L. Jones, et al.."Photofission-based, Nuclear Material Detection: Technology Demonstration." INL Formal Report, INEEL 02-01406, December 2002.

4. "Better Than Dogs," Need to Know, INL National Security Newsletter, 3, 2, p.6 (January 2003)

5. Active Interrogation Workshop, INL Publication, Idaho Falls, Idaho, June 29-30, 2004.

6. J.L. Jones, “Active, Non-intrusive Inspection Technologies," Sixth International Meeting on Nuclear Applications of Accelerator Technology (AccApp’03)," ANS Conference Publication, p.33 (June 1-5, 2003)

7. J.L. Jones, et al., "Photonuclear-based, Nuclear Material Detection System for Cargo Containers," $8^{\text {th }}$ International Conference on the Application of Accelerators in Research and Industry, Fort Worth, Texas, October 11-15, 2004.

8. $\quad$ S.S. Dietrick and B.L. Berman. "Atlas of Photoneutron Cross Sections Obtained with Monoenergetic Photons,” UCRL-94820, June 1986.

9. J.L. Jones, et al., "Proof-of-Concept Assessment of a Photofission-Based Interrogation System for the Detection of Shielded Nuclear Material,” INL Formal Report, 00-01523, November 2000.

10. J.S. Hendricks, et al., "MCNPX, Version 2.5.e,” LANL Report, LAN-UR-04-0569, February 2004.

11. J.L. Jones, et. al., "ARACOR Eagle-matched Operations and Neutron Detector Performance Tests,” INL Formal Report, INEEL/EXT-02-00823, June 2003.

12. Letter report, J.L. Jones to A. Feinberg, "Project Deliverable: Photonuclear-based, Infield Assessment of the Latest ARAOR Eagle Inspection System Design," INL Letter, CCN 51431, July 29, 2004. 
Appendix A

PPND Performance Testing at HPIL 


\section{Appendix A \\ PPND Performance testing at HPIL}

The Pulsed Photonuclear Neutron Detectors (PPNDs) were tested to determine their neutron efficiency using a National Institute of Standards and Technology (NIST) traceable ${ }^{252} \mathrm{Cf}$ source at the Health Physics Instrument Laboratory (HPIL). The tests were conducted in a room specially designed to have low and uniform neutron scatter. Results from the tests indicate that the PPND's intrinsic efficiency is about $5.9 \%$ for ${ }^{252} \mathrm{Cf}$ and the absolute efficiency was $1.6 \times 10^{-4}$ per source neutron at 1.5 meters. The details of these tests configurations, sources, and counting equipment are provided below.

\section{Test Configuration Setup:}

The general test configuration with the source centered in the middle of the room is shown in Figure A-1. This source location provides about 5 meters between the concrete floor and source and about 5 meters between the concrete ceiling and the source. Also the diameter of the cylindrical room is about 10 meters providing 5 meters between the source and the concrete wall. To access the test area an aluminum grating is provided 1 meter below the source location. The PPNDs (without shrouds) were horizontally positioned on the calibration tables so that the detector center was aligned with the neutron source centerline. The facility laser alignment system was used to position each detector as outlined above. This positioning scheme places the center of the active region (both vertically and horizontally) at the elevation center of the neutron source. The distance between the radioactive source and each detector was 1.5 meters.

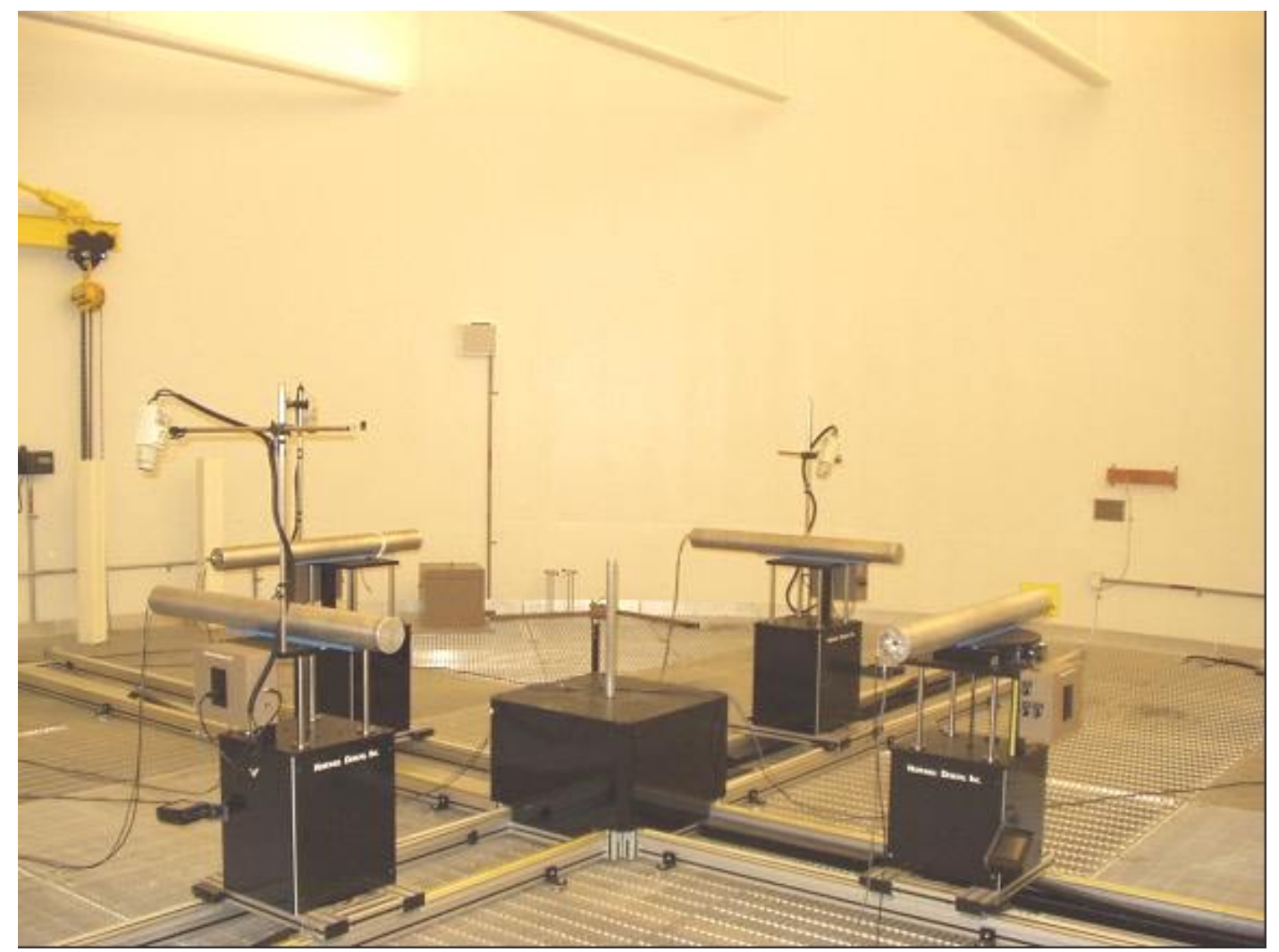

Figure A-1. Detector placement relative to the radioactive source. 


\section{Sources:}

Three neutron sources were used for testing with emission rates presented in Table A.1. Based on the NIST calibration certification date, the sources were decayed to the test date using a 2.646-year halflife to determine the neutron emission rate for the day of these tests. Although all three sources were used for a quick count rate sensitivity study, only the small source ( $4.3 \times 10^{7}$ neutrons per second) was used for the efficiency tests. Even the smallest source provided more than three orders-of-magnitude more count rate than desired. The count rate that was observed from the lowest source was about two orders-ofmagnitude larger that the count rate observed when inspecting a 4.4-kg plate of unshielded depleted uranium using a nominal 8-MeV electron beam operation. Based on the count rate sensitivity study, the National Instruments PXI system did not have any counting loss even with the large sources.

Table A-1. NIST-calibrated neutron emission rates decayed to test date.

\begin{tabular}{cccc} 
NIST $n / s$ & Decayed NIST n/s & Cert Date & Use Date \\
\hline $6.28 \mathrm{E}+09$ & $4.13 \mathrm{E}+09$ & $12 / 24 / 2002$ & $7 / 29 / 2004$ \\
$6.03 \mathrm{E}+08$ & $3.75 \mathrm{E}+08$ & $10 / 7 / 2002$ & $7 / 29 / 2004$ \\
$6.74 \mathrm{E}+07$ & $4.30 \mathrm{E}+07$ & $11 / 9 / 2002$ & $7 / 29 / 2004$ \\
\hline
\end{tabular}

\section{Test Equipment:}

Two counting systems seen in Figure A-2 were the multi-channel scaler (MCS) and the FPGA LabView RIO/PXI system. A graphical depiction of the resulting data is presented in Figure A-3.

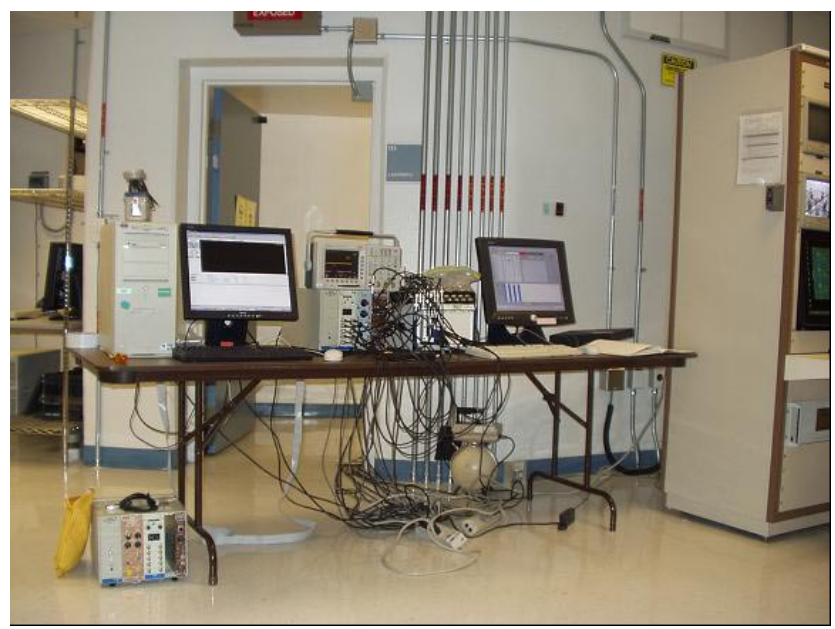

Figure A-2. MCS and PXI counting systems. 


\section{Test Data:}

Two-minute count times were used and about 800,000 counts/test were accumulated. The count rate for an average detector was about 7,000 counts per second.
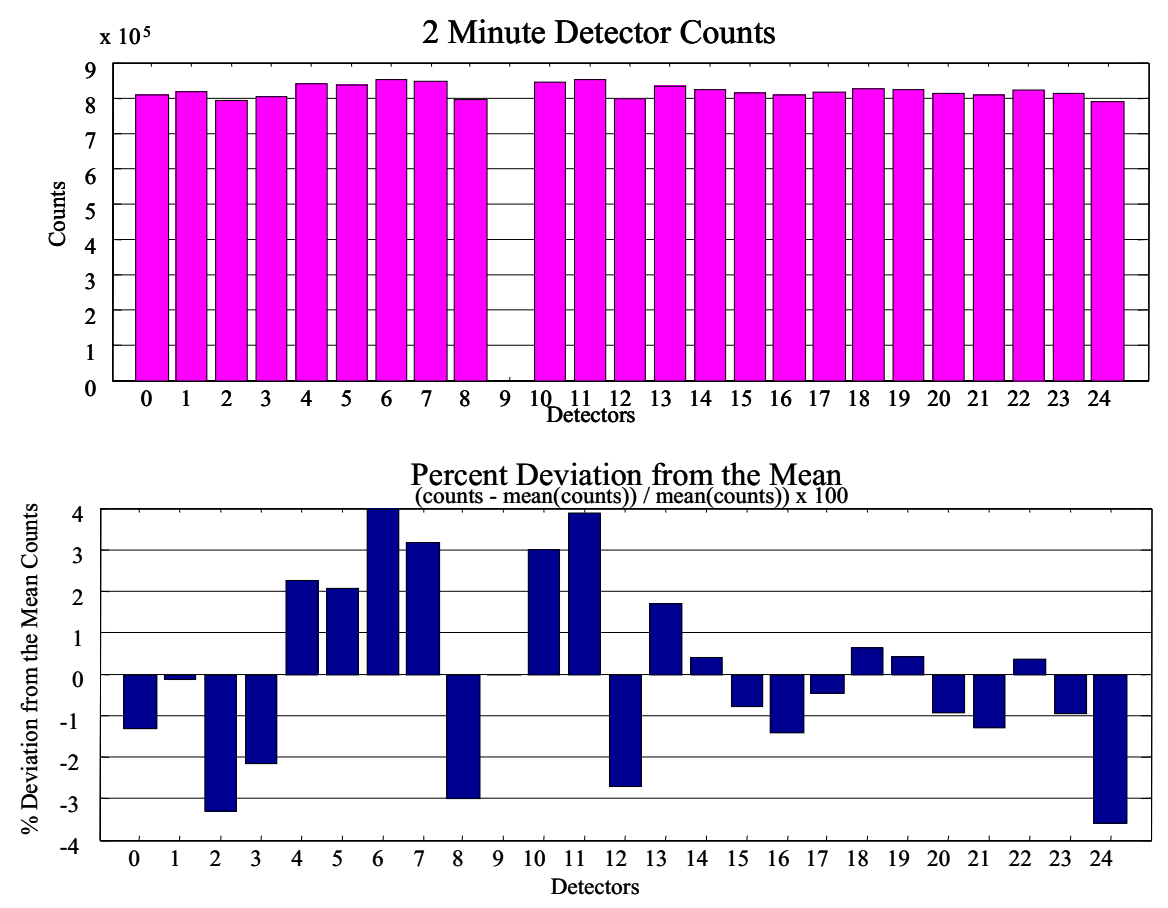

Figure A-3. Individual PPND test results. 
Appendix B

\section{Base Pallet Construction}




\section{Appendix B \\ Base Pallet Construction}

The measured base pallet weight is $84.5 \mathrm{lbs}$. The pallet, shown in Figure B-1, is constructed of three 4 × 4 ( 3.5 in. x 3.5 in.) boards, five $2 \times 10$ (1.5 in. x 9.5 in.) boards, and two $2 \times 4$ (1.5 in. x 3.5 in.) boards. The corners of the base pallet are cut off to allow room for the legs of the aluminum stacking tables (constructed for stacking) to slip down over the base pallet and still fit within a required 40 in. $x 48$ in. footprint. All lumber used in construction is pine.
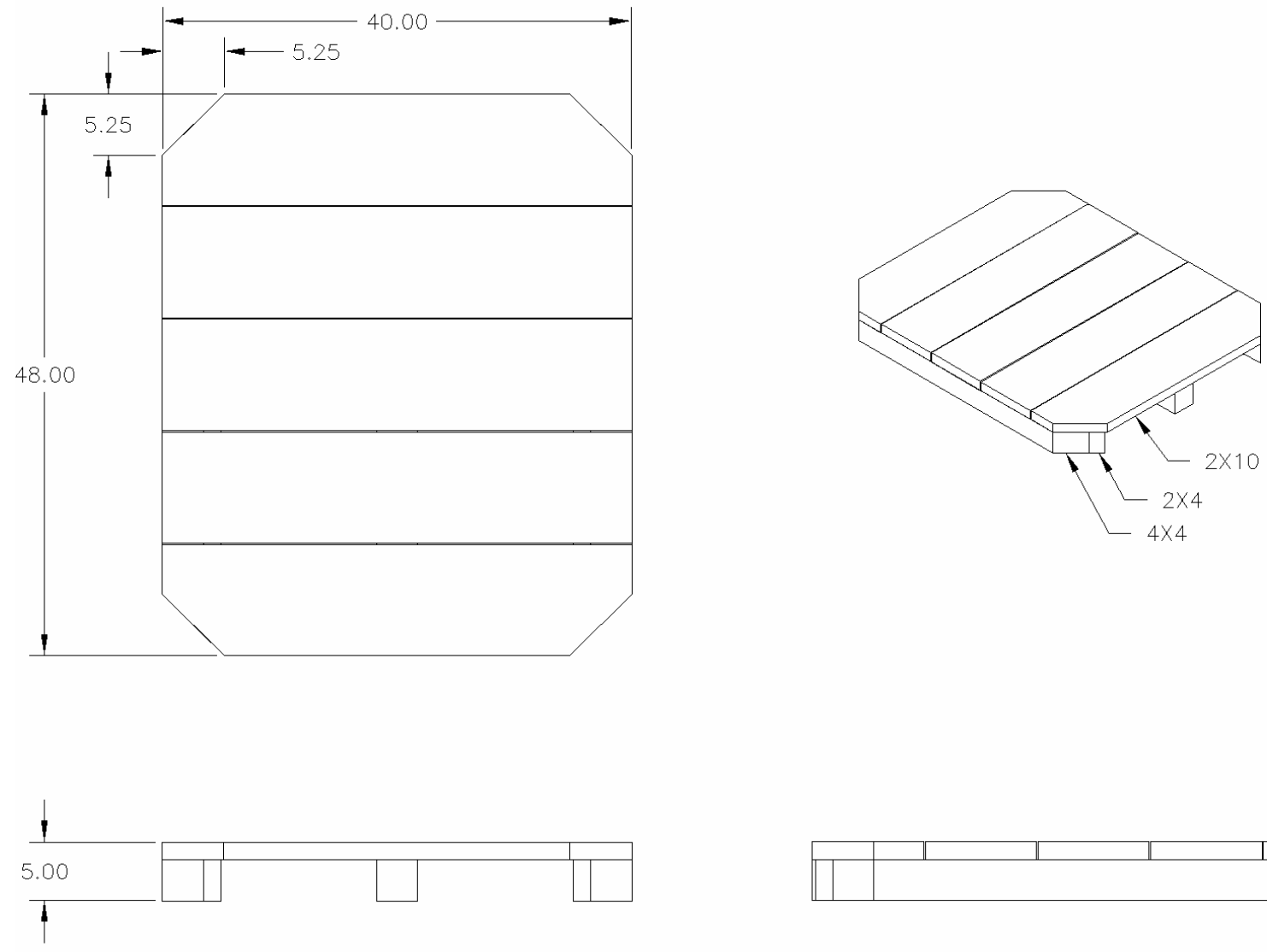

Figure B-1. Pallet base.

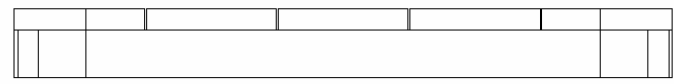


Appendix C

Stacking Frame 


\section{Appendix C \\ Stacking Frame}

Four aluminum tables have been fabricated to allow stacking of calibration pallets (one on top of another) within almost any standard cargo transport container. The tables, shown in Figure $\mathrm{C}-1$, are designed to support at least $3500 \mathrm{lbs}$ and to fit within the required 40 in. $\mathrm{x} 48 \mathrm{in}$. footprint. Each table weighs $212 \mathrm{lbs}$.
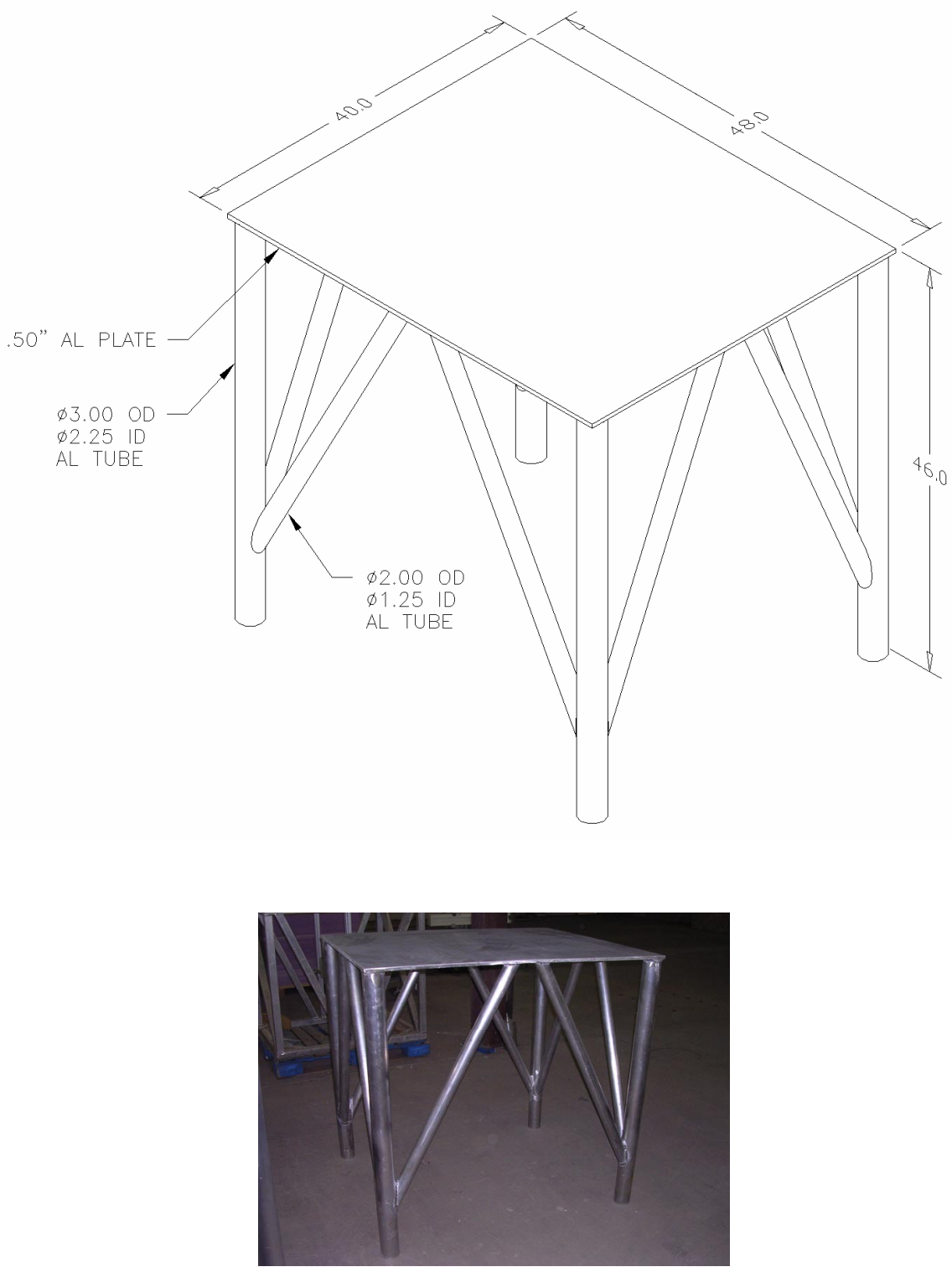

Figure C-1. Pallet stacking table. 
Appendix D

"Empty" Pallet 


\section{Appendix D \\ "Empty" Pallet}

The pedestal for the "Empty" Calibration Pallet, shown Figure D-1, consists of a 15 in. $x 15$ in. $x$ $1 / 4$ in. base plate, a 15-1/2 in. tall pipe, and an 8" O.D, 8" high can. All components of the pedestal are constructed of 0.5-inch-thick aluminum. The total weight of the Empty Calibration Pallet assembly is $101.9 \mathrm{lbs}$ total (84.5 lbs for the pallet [See Appendix B], $16.4 \mathrm{lbs}$ for the pedestal).
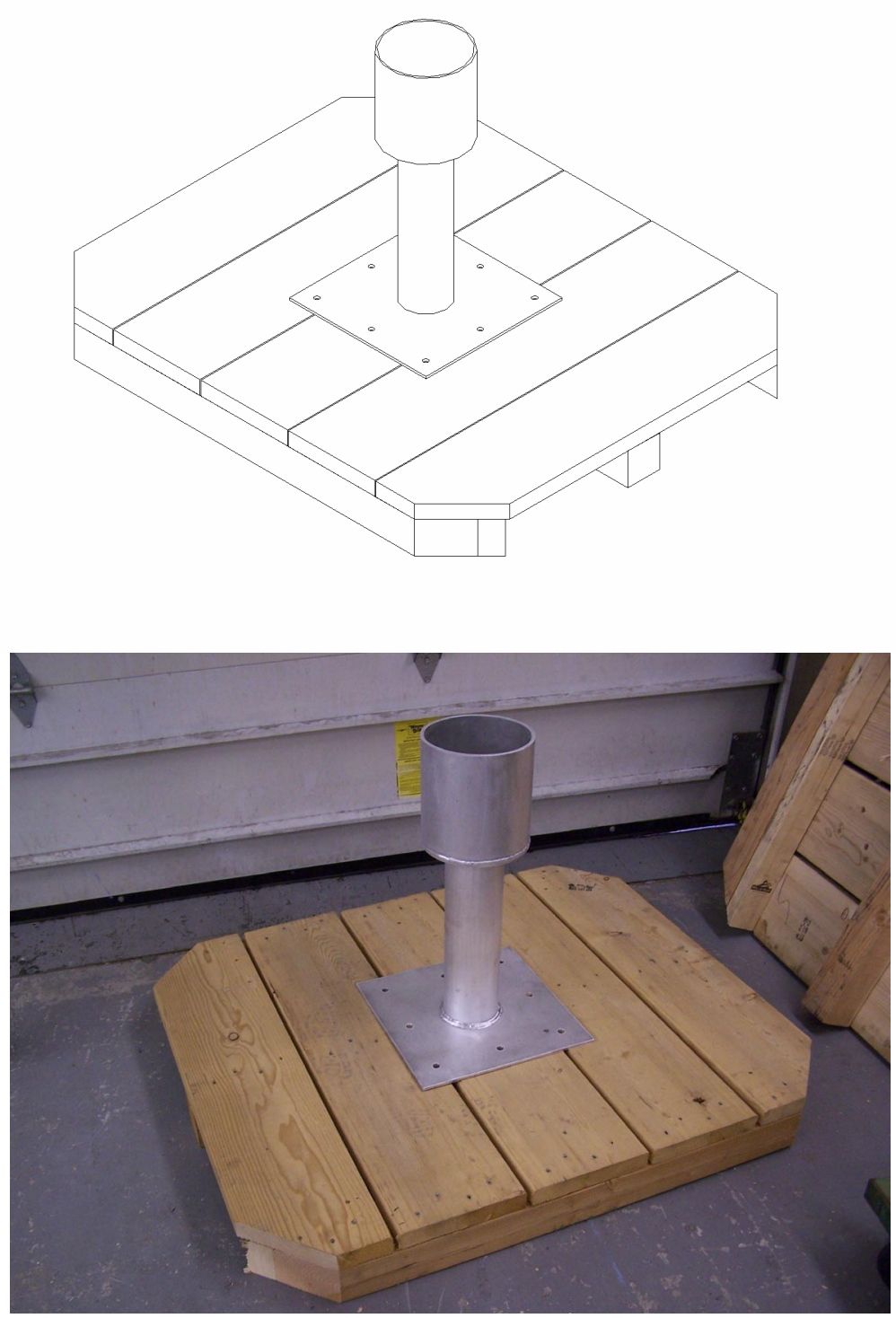

Figure D.1. Complete "Empty" Pallet design. 


\section{Appendix E \\ Celotex, Wood, Polyethylene and Borated-Polyethylene Pallet}




\section{Appendix E \\ Celotex, Wood, Polyethylene and Borated-Polyethylene Pallet}

These Calibration Pallets measure 42 in, (L) x 34 in. (W) x 40 in. (H) and sit upon a base pallet as shown in Appendix B. The primary material consists of forty 1-in thick sheet layers, with eight of those layers modified to form an 8-in. diameter, 8-in. high cavity in the center of the material stack. A removable plug of material allows side access to the pallet for inclusion of nuclear material. The assembled configuration with its removable plug is shown in Figure E-1. Figure E-2 shows the main sheet configurations of the stacked layers, and Figure E-3 presents the actual Calibration Pallets represented by this Appendix.

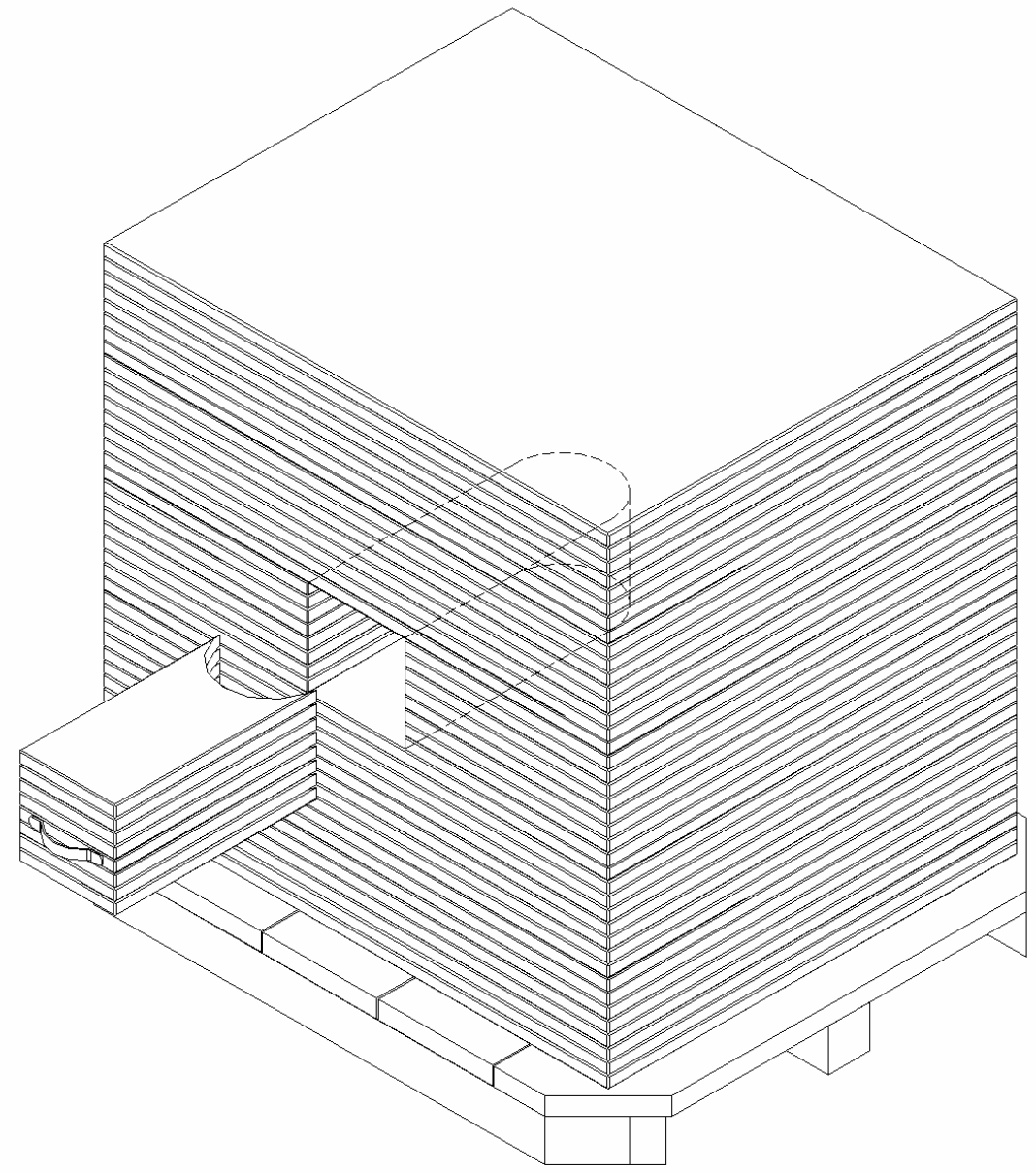

Figure E-1. Basic Calibration Pallet Design. 


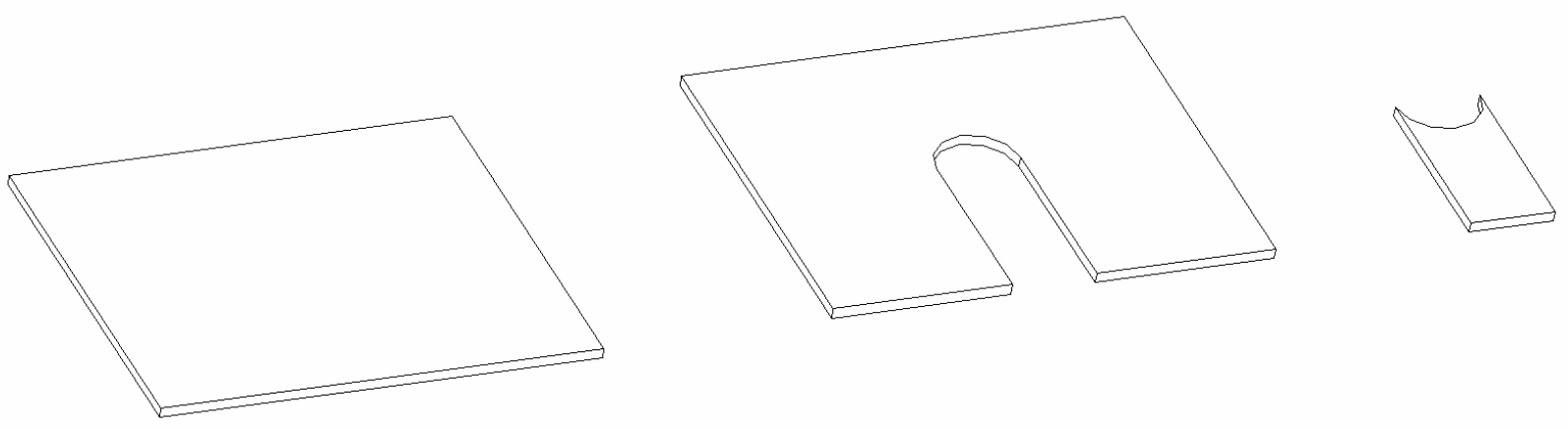

Figure E-2. The main sheet configurations of the stacked pallet layers.
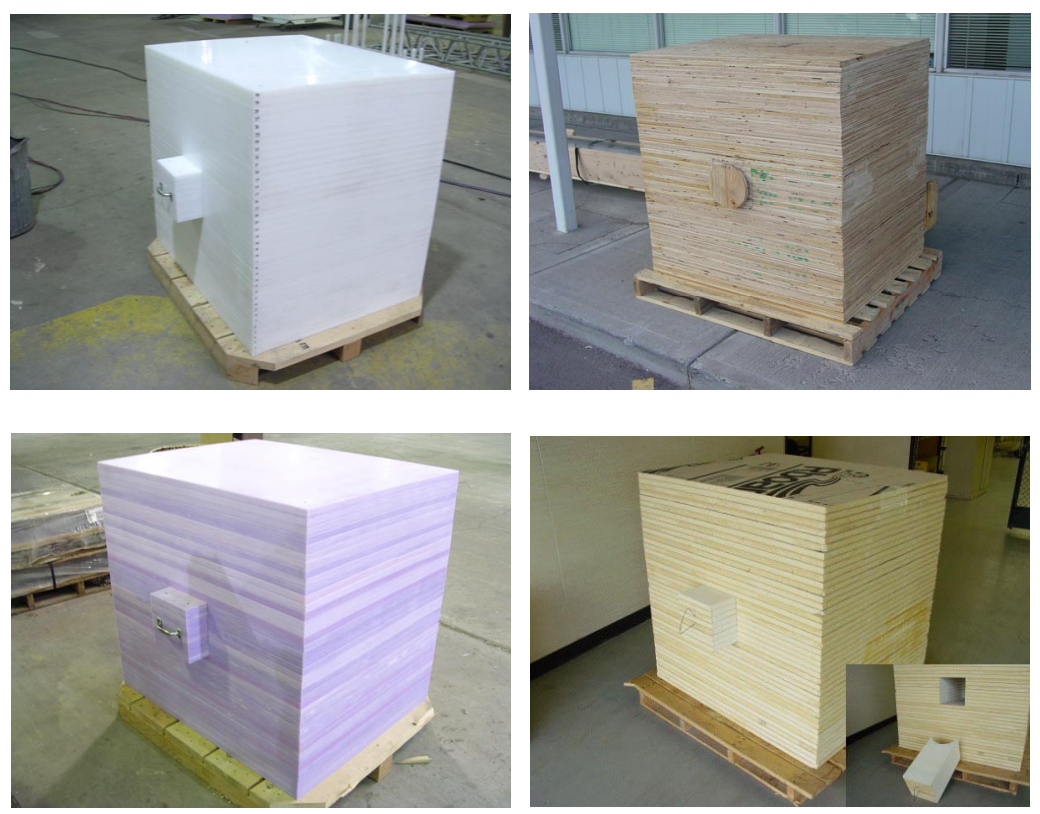

Figure E-3. Actual Plywood, Celotex, Polyethylene, and Borated Polyethylene Calibration Pallets. 
Appendix F

Lead Pallet 


\section{Appendix F Lead Pallet}

The lead pallet consists of a pallet base (Appendix B), an aluminum pedestal supporting a 0.5 -inchthick aluminum container housing an 8-in. diameter, 8-inch tall void completely surrounded on the bottom and sides with a 2-inch-thick lead shield, and the associated 2-inch thick lead lid (allowing access to the void space). This complete configuration design is shown in Figure F-1 and weights $557.5 \mathrm{lbm}$. Figure F-2 and F-3, and F-4 show details of the pedestal, container, and lid, respectively.
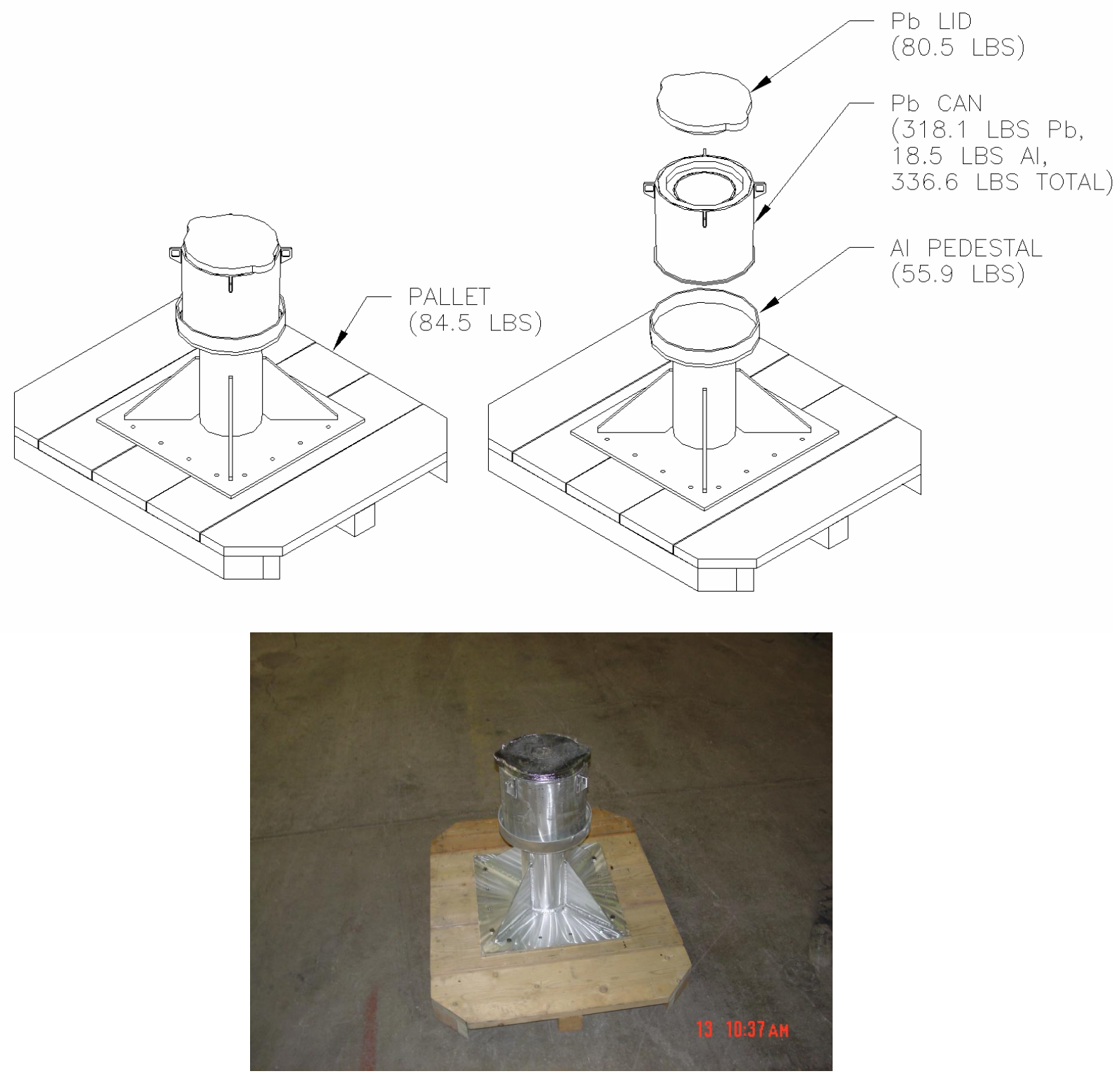

Figure F-1. Complete Lead Calibration Pallet design. 


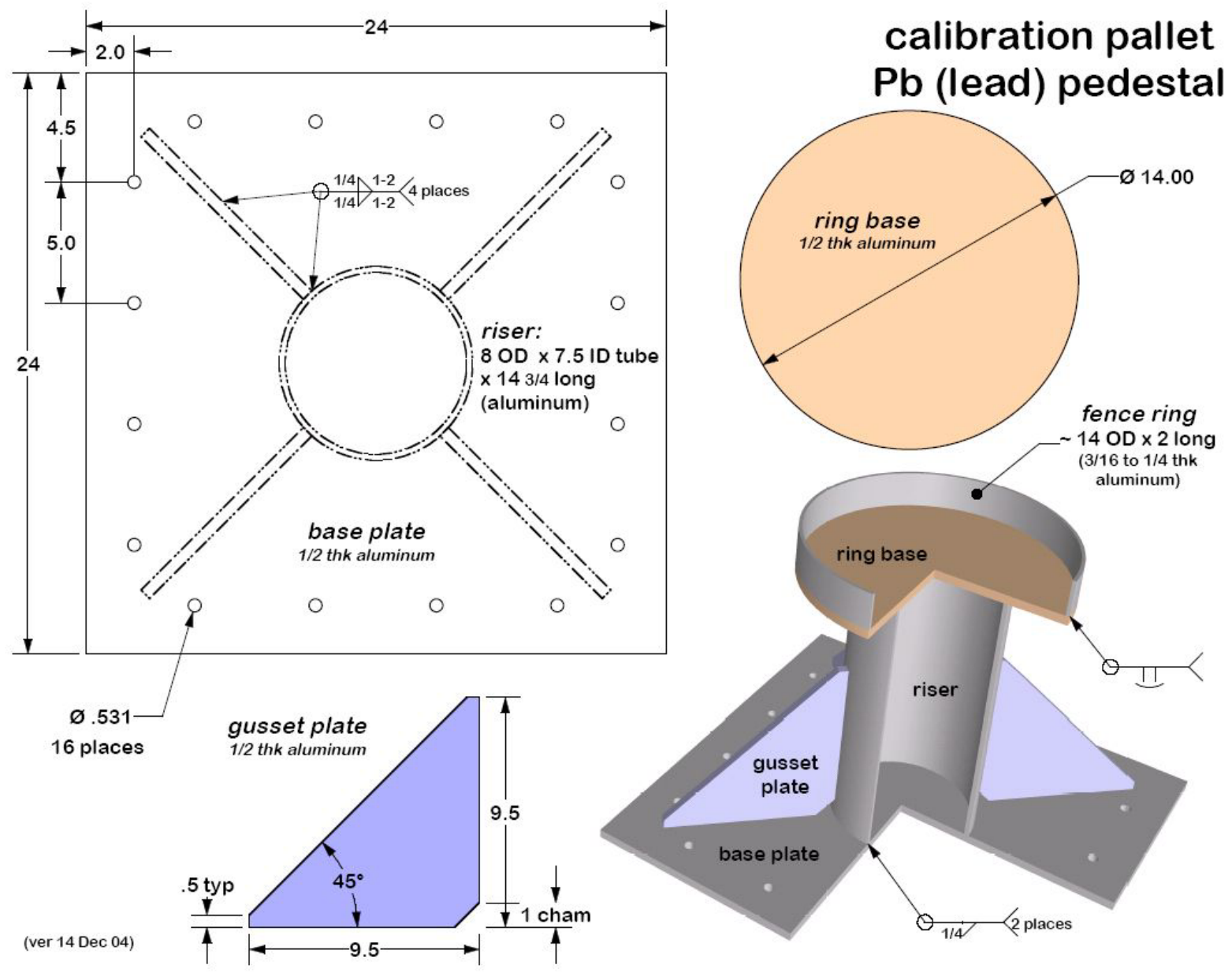

Figure F-2. Pedestal design for the Lead Calibration Pallet. 


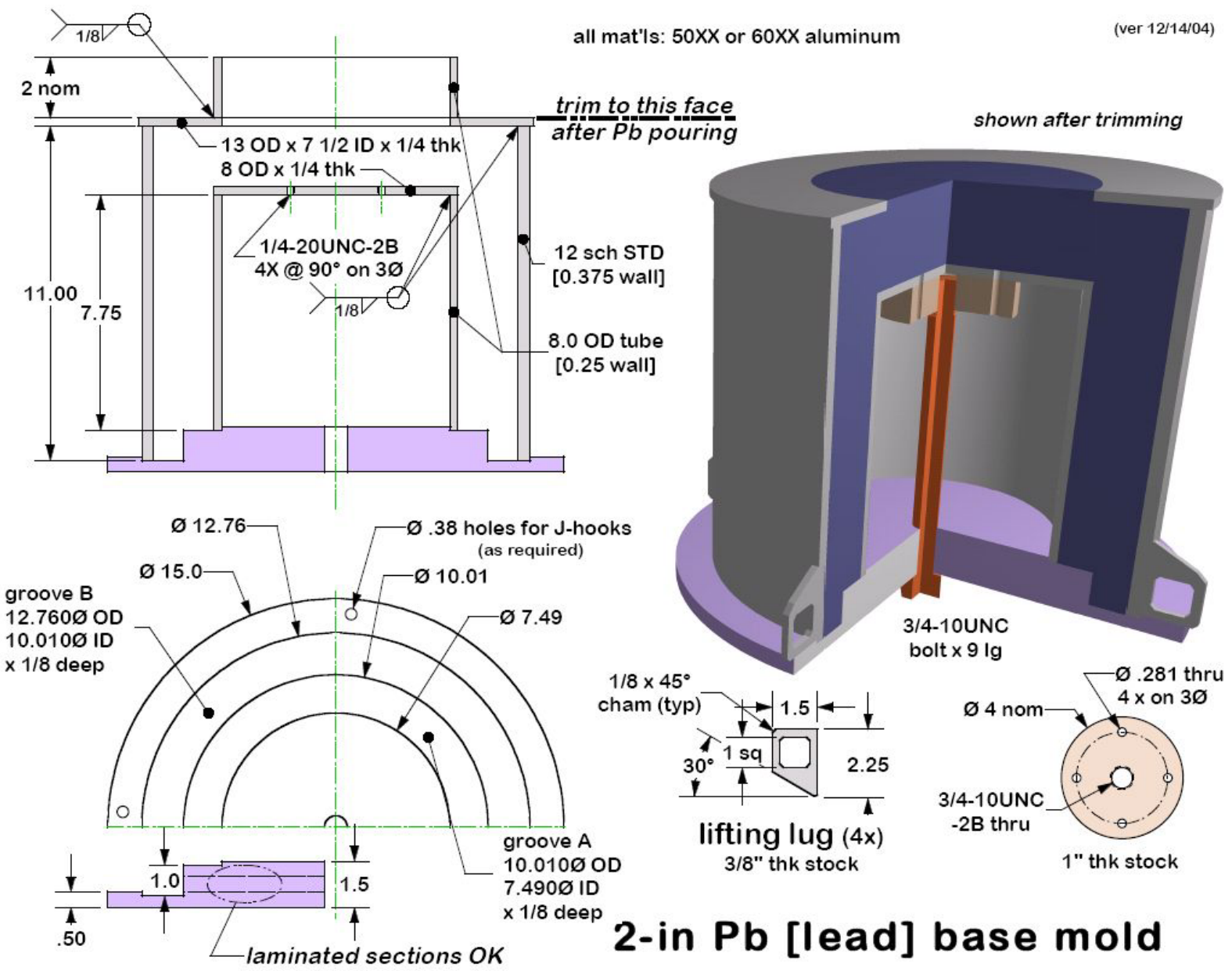

Figure F-3. Container design for Lead Calibration Pallet. 

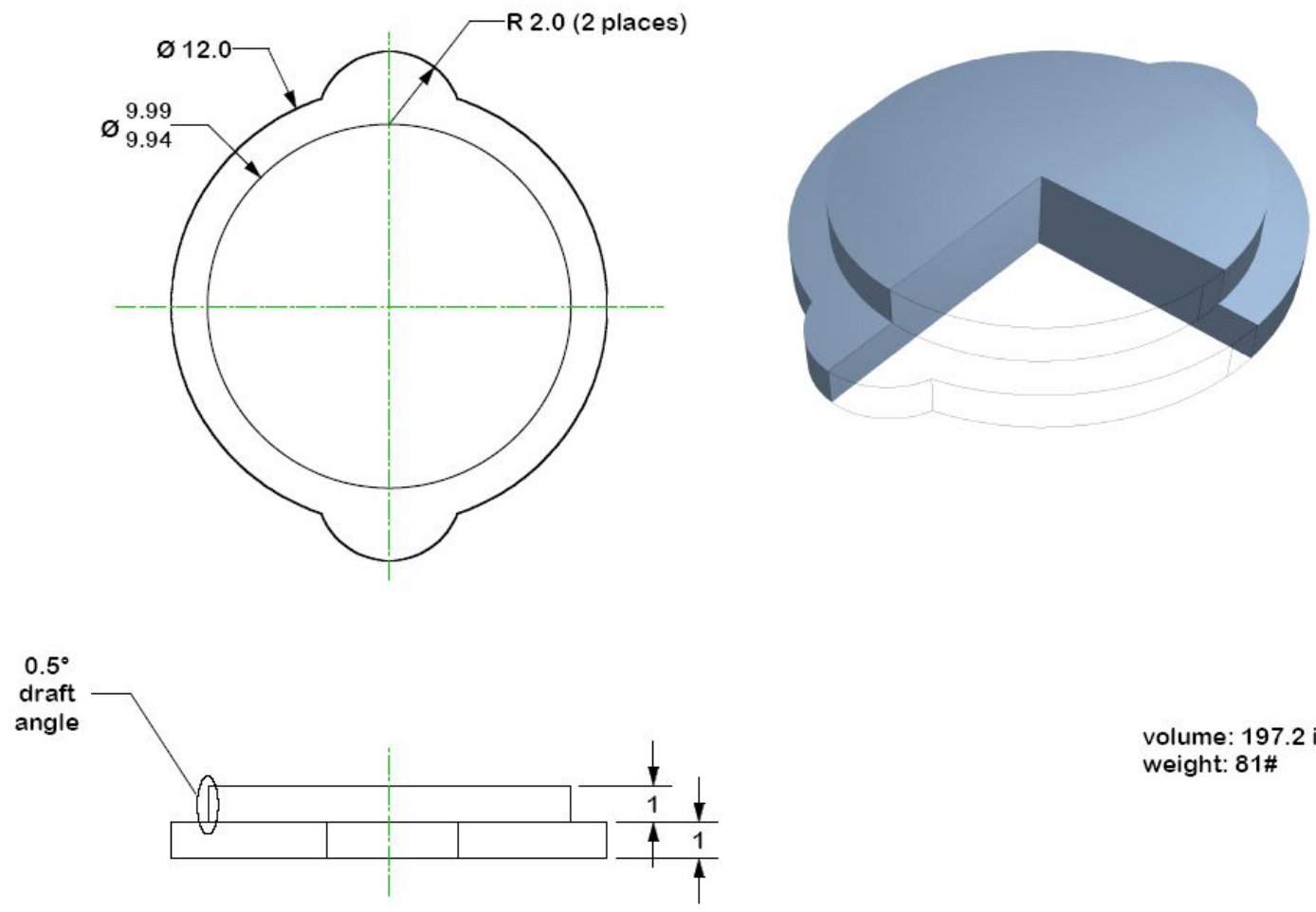

volume: $197.2 \mathrm{in}^{\wedge} 3$

weight: 81\#

\section{2-in Pb [lead] lid}

Figure F-4. Container lid for Lead Calibration Pallet. 


\section{Appendix G}

\section{Iron Pallet}




\section{Appendix G \\ Iron Pallet}

The Iron Calibration Pallet, shown in Figure G-1, consists of the base wood pallet, a 0.5 -in.-thick aluminum pedestal (see Figure G-2), an 8 in. diameter, 8 in. high cavity formed by a base, annular ring \#1 and the lid, and a series of annular carbon steel rings that cumulatively sum to a total thickness of $6-1 / 8$ in.
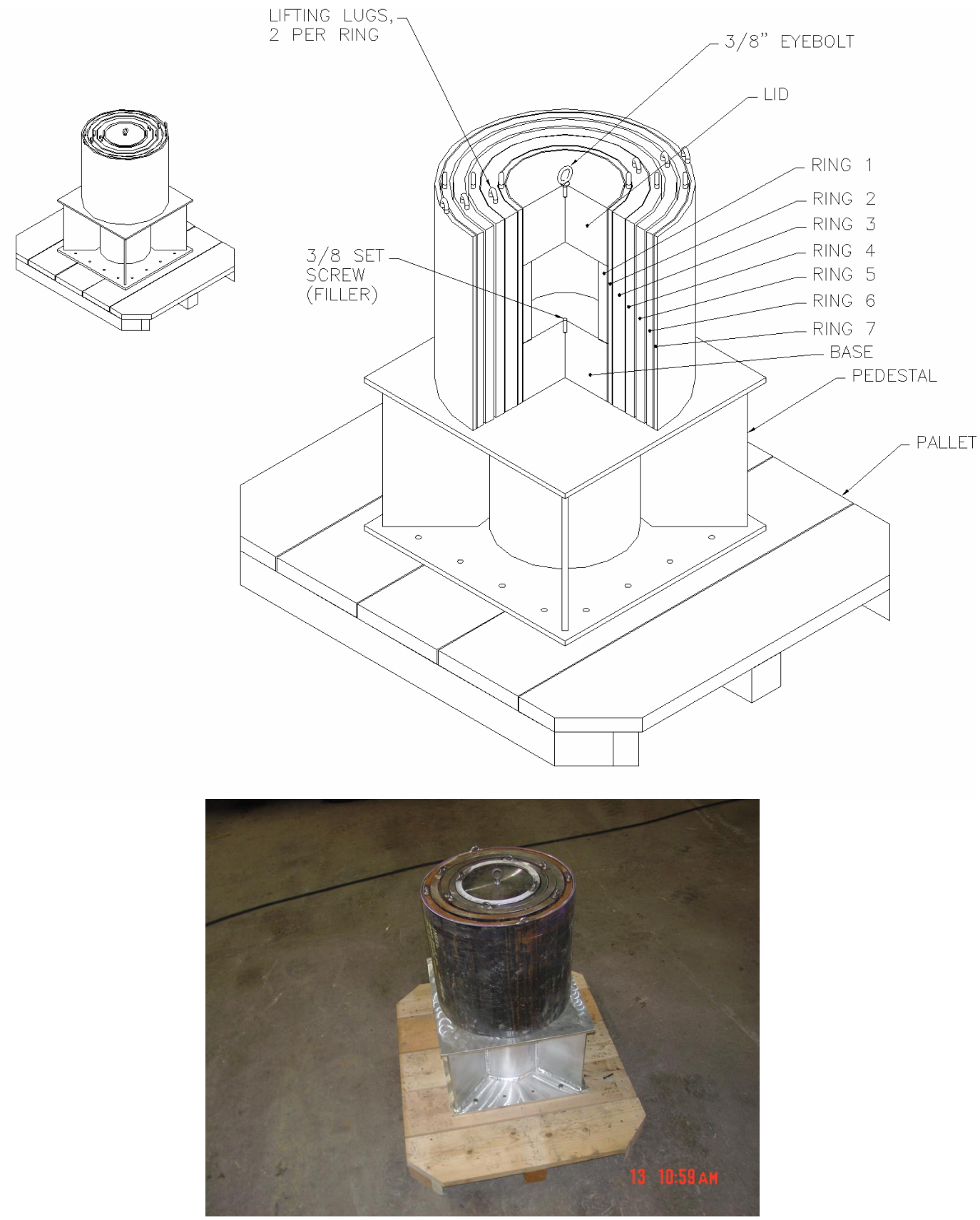

Figure G-1. The complete Iron Calibration Pallet design. 

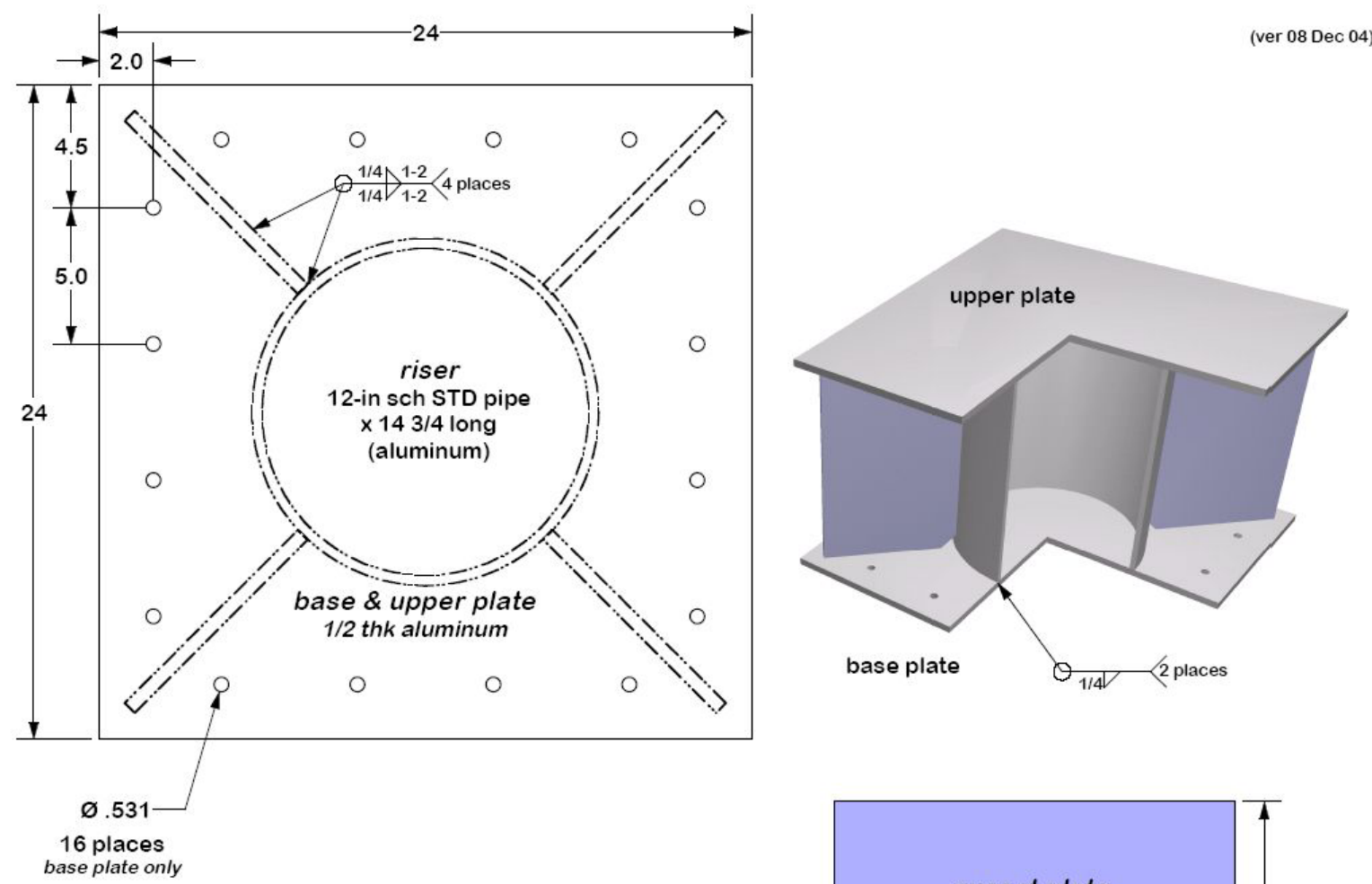

\section{calibration pallet Fe (iron) pedestal}

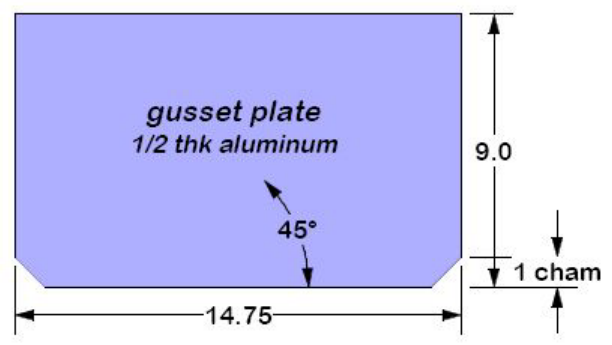

Figure G-2. The pedestal for the Iron Calibration Pallet. 
Appendix $\mathrm{H}$

\section{The "Challenge" Pallet}




\section{Appendix H The "Challenge" Pallet}

The borated Polyethylene/lead (B Poly/Pb) Calibration Pallet (See Figure H-1) consists of the base pallet, 48 sheets of 1 in. thick Borated Poly, 24 of those sheets having a 24 in. x 24 in. hole cut out of them to accommodate installation of standard 2 in. $x 4$ in. $x 8$ in. lead bricks in the middle of the assembly. The lead bricks (208 of them!) are arranged to minimize any streaming, to form a 24 in. x 24 in. $x 24$ in. assembly at the center of the assembly, and to allow for a 8 in. $x 8$ in. $x 8$ in. central opening (See Figure H-2). An 8-inch-diameter, 0.5-in.-thick aluminum can is installed into the chamber to provide support for the lead bricks above the chamber. Hence, this configuration (rightfully named the "Challenge" Pallet) permits a nuclear material to be completely surrounded by 8 inches of lead and then enclosed by 12 inches of borated polyethylene. Due to the overall mass, no access plug was included in his design; hence, nuclear material addition/removal must be accompanied with a partial pallet unloading process. To facilitate unloading, the pallet design was broken down into three sections to allow for moving components of lower weights. Figure H-3 shows some of these sections. 

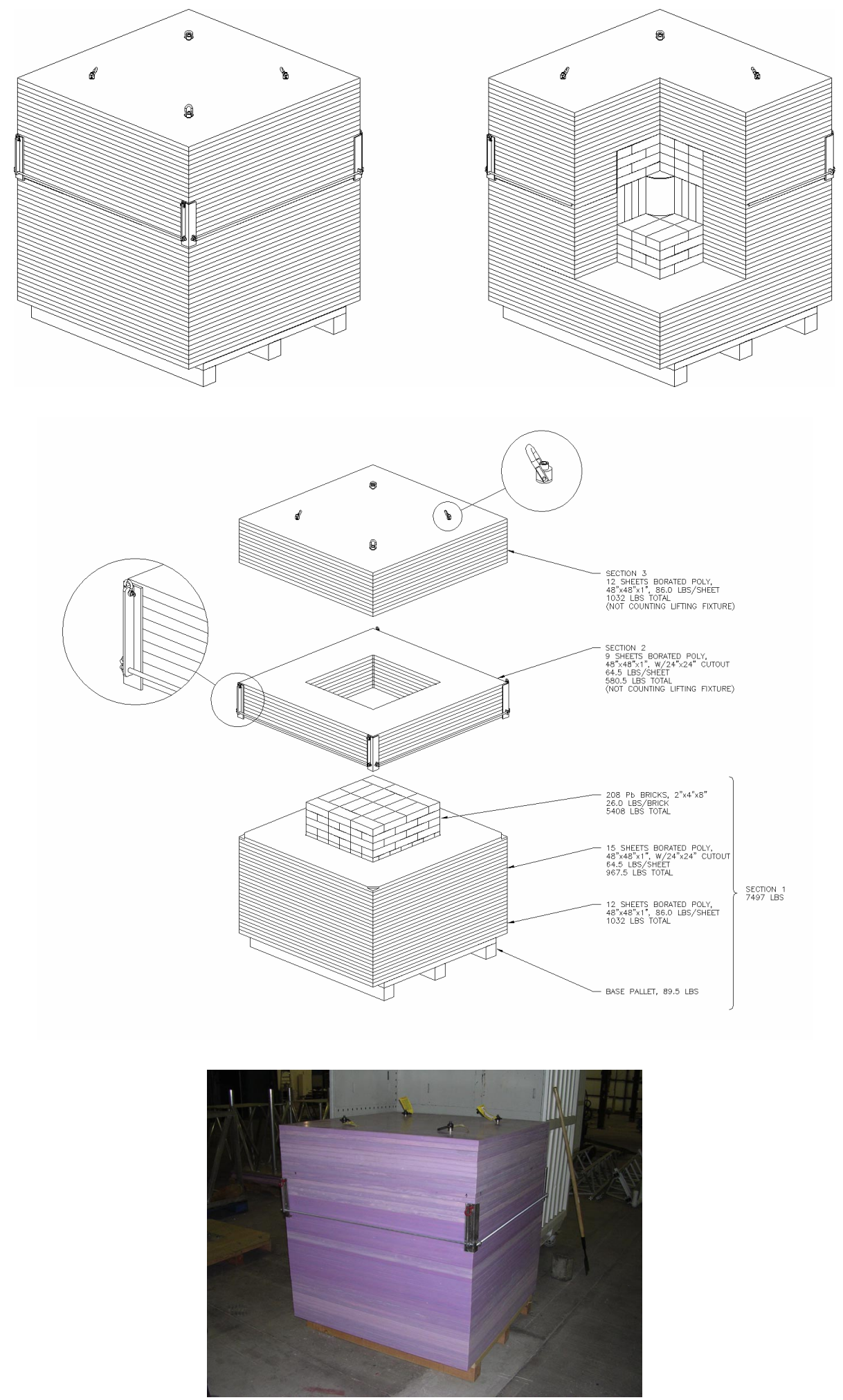

Figure H-1. Complete "Challenge" Pallet design. 
The lead central region consists of standard $2 \times 4 \times 8$ in. lead bricks. The brick arrangement is shown in the Figure H-2 below with layer \#1 being bottom (lower most) layer.

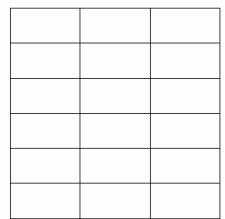

1

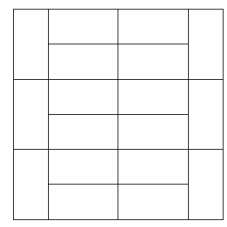

2

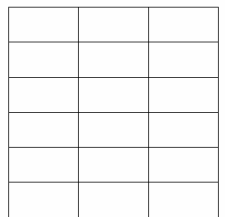

3

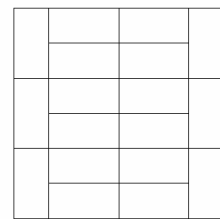

4

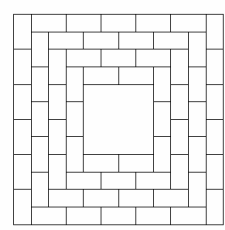

5

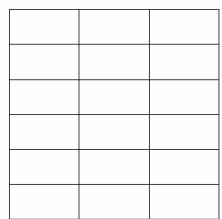

6

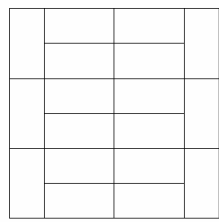

7

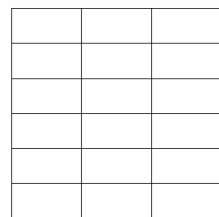

8

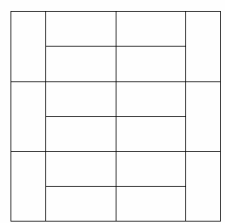

9

Figure H-2. Standard lead brick layout of the central region of the "Challenge" Pallet.

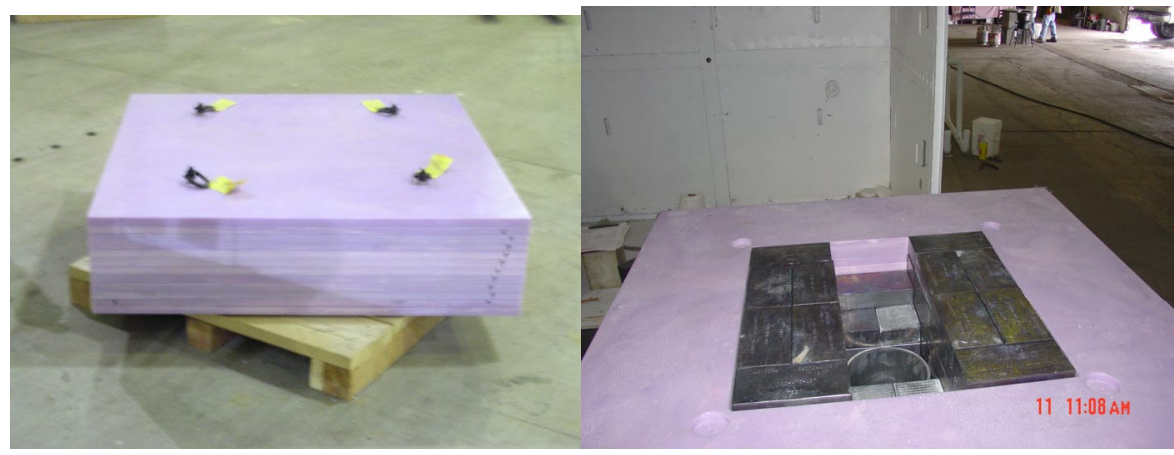

Figure H-3 Partially-assembled "Challenge” Pallet sections (Top section on left). 